\title{
Estudos sobre Teorias com Chern-Simons: Equivalência entre Modelos e Efeito Aharonov-Bohm Não-Abeliano
}

\author{
Luís Carlos Malacarne
}

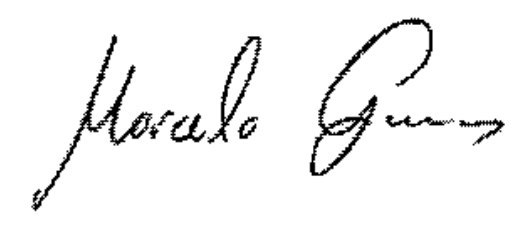

ORIENTADOR: Prof. Dr. Marcelo Otavio Caminha Gomes

\section{BANCA EXAMINADORA}
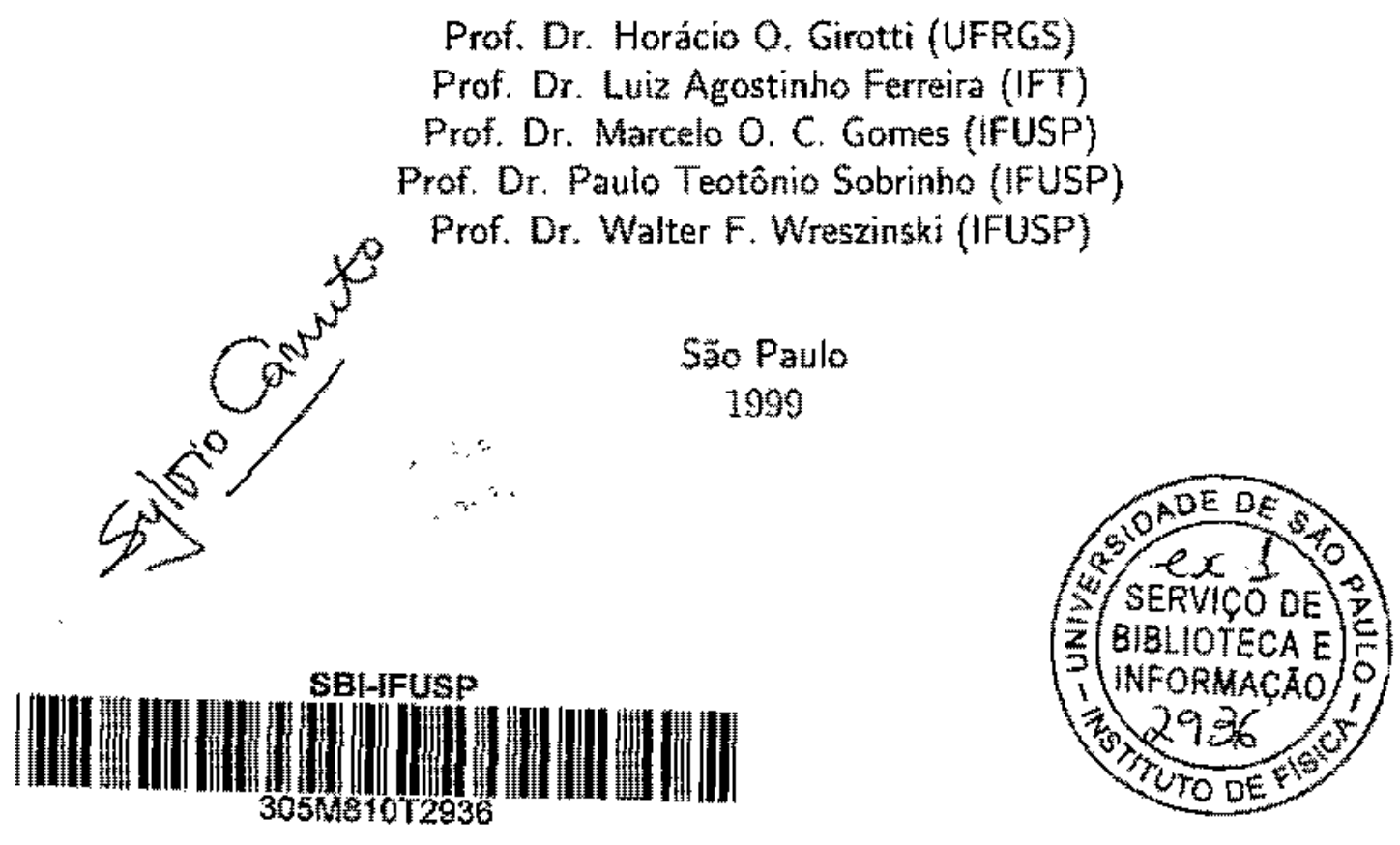

Tese de Doutorado

submetida ao Instituto de Física da Universidade de \$ăo Paulo 


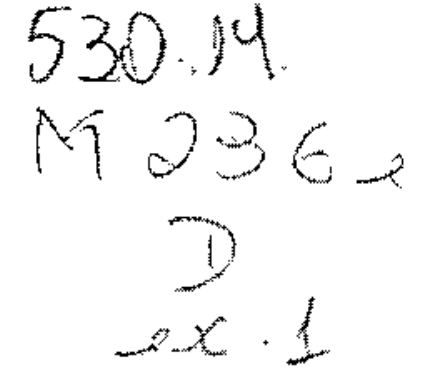

FICHA CATALOGRÁFICA

Preparada pelo Serviço de Biblioteca e Informação do Instituto de Fisica da Universidade de São Paulo

Malacarne, Luis Cartos

Estudos sobre Teorias com Chern-Simons: Equivaléncia entre Modelos e Efeito Aharonov-Bohm não-Abeliano. São Paulo, 1999.

Tese (Doutoramento) Universidade de Săo Paulo. instituto de Fisica - Departamento de Fisica Matemática

Orientador: Prof. Dr. Marcelo Otavio Caminha Gomes Área de Concentração: Fisica de Particulas Elementares e Campos

Unitermos: 1. Teoria de Campos em (2+1) Dimensões;

2. Teoria de Chern-Simons; 3. Efeito Arahonov-Bohm;

4. Modelos de Maxwell-Chern-Simons e Self-Dual. 


\section{ABSTRACT}

The aim of this work ts to study some models with Chmm-Simons term, which induces soveral properties such as topological mass and fractional spin. We start studying the exact equivalence between the Self-Dual model minimally coupled to a Dirac field and the Maxwell-Chern-Simons model with non-minimal magnetie coupling to fermions. We show that the fermions sectors of the models are equivalent only if a Thirring like interaction is included. Next we analyze the non-Abelian Aharonow-Bohn scattaring or 0 and $1 / 2$ spin particles. Starting from the relativistic tuantum feld theory and using a Coulonb gauge formalism, the one loop renormalization is implemented. Trough the introduction of an intermediary cutof, separating the regions of high and low integration momenturn, the nontelativistic limit is derived. In this approach quantun field theory vacuum polarization and anomalous magnetic momentum are automatically incorporated. We also study some properties of the charged one spin particles interacting with Chern-Simons field. 


\section{RESUMO}

O objetivo desta tese é estudar alguns modelos contendo o termo de Chern-Simons, cuja presença induz várias características tais como massa topológica e spin fracionário. Iniciamos tratando o problema da equivalênei entre os modelos Auto-Dual minimamente acoplado com o campo de Dirac, e o modelo Maxwell-Chern-Simons corn um acoplamento magnético com férmions. Nós mostramos que os setores fermiônicos dos modelos somente sâo equivalentes se adicionarmos uma auto-interaçăo tipo Thirring. En seguida analisamos o efeito Aharonov-Bohm näo-Abeliano para particulas de spin 0 e 1/2. Partindo da teoria quântica de campos relativistica no gauge de Coulomb e usando o procedimento do corte intermediánio, para separar o monento de integração nas regiós de baixa e alta energin, nós obtemos o limite năo relativistico dos modelos. Desta forma as correçôs radiativas, tais como polarizaçăo do vácuo e correçăo de vértice, são zutomaticamente inclutdas. Finalizando estudamos algumas propriadades de modelos descrevendo partículas carregadas de spin 1 interagindo com o campo de Chern-Simon. 
Dedicatória, Aos Meus Pais e a Rosângela. 


\section{Agradecimentos}

- Ao Prof. Dr. Aarcelo Otavio Caminha Gomes pela orientação;

- Aos meus pais e irnäos: Aurélio, Lúcia, Gilmar, Neodir, Ademar, Ademir e Marilene;

- A Rosängela, Isabela, Olga e Francisco:

- Aos amigos, em especial Rênio, Sérgio, Van Súrgio:

- Ao Prof. Adilson J. da Silva:

- Ao Departamento de Fisiea da Universidade Estadual de Maringá:

- Ao Departamento de Fisica Matemática do Instituto de Física da USP:

- Ao CNPq e CAPES pelo apoio financeiro. 


\section{Conteúdo}

Introdução

1 Equivalência entre os Modelos Auto-Dual e Maxwell-Chern-Simons Acoplados com Férruions 12

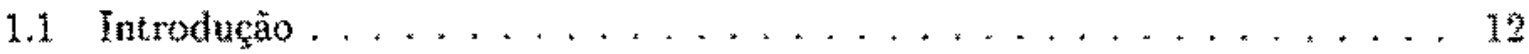

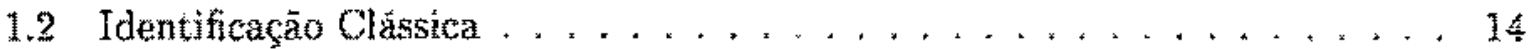

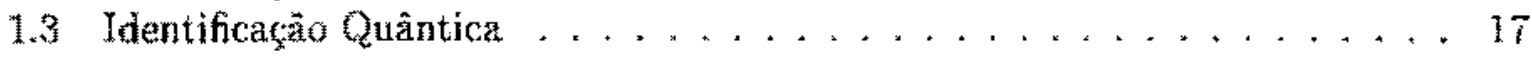

2 Espalharnento Aharonov-Bohm Não-Abeliano para Partícula Escalar 22

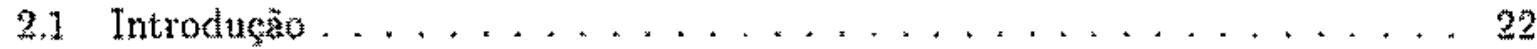

2.2 Nodelo Não Relativístico . . . . . . . . . . . . . . . . 23

2.3 Modelo Relativístico . . . . . . . . . . . . . . . 26

2.3.1 Correçào para A Auto-Energia . . . . . . . . . . . . 29

$2.3,2$ Tensor de Polarizą̧äo $\ldots \ldots \ldots \ldots \ldots \ldots \ldots$

$2,3.3$ Correção de Vêrtice. . . . . . . . . . . . . . 32

2.3.4 Espalhamento Partícula-Particula . . . . . . . . . . 33

2.4 Análise . . . . . . . . . . . . . . . . . . 44

3 Espalhamento Aharonov-Bohm Nāo-Abeliano para Particula de Spin $1 / 2 \quad 52$

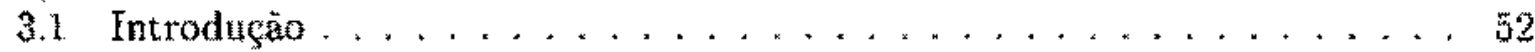

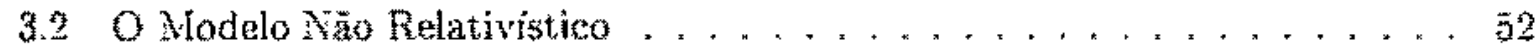

3.3 O Modelo Relativistico . . . . . . . . . . . . . . . . . 57

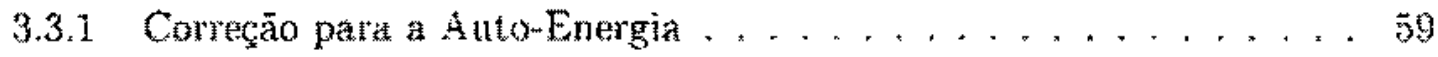

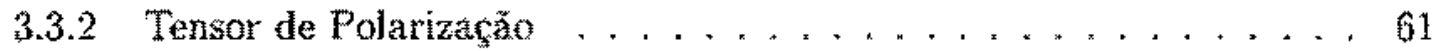

3.3 .3 Correção de Vértice . . . . . . . . . . . . . . . 03

3.3 .4 Espalhamesto en 1-Loop . . . . . . . . . . . . 65

3.4 Arthise . . . . . . . . . . . . . . . . . 70

4 Particula Massiva de Spin 1 Interagindo com Chern-Simons 72

4.1 Introdução . . . . . . . . . . . . . . 72

4.2 Vetores de Polarização e Regras de Feynman . . . . . . . . . 73 
4.2 .1 Modelo de Proca $\ldots \ldots \ldots \ldots \ldots$

4.2 .2 Modelo de Maxwell Chern-Simons . . . . . . . . . . 78

4.3 Correçăo de $1-1$ oop $\ldots \ldots \ldots \ldots \ldots$

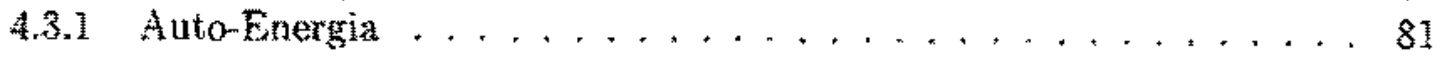

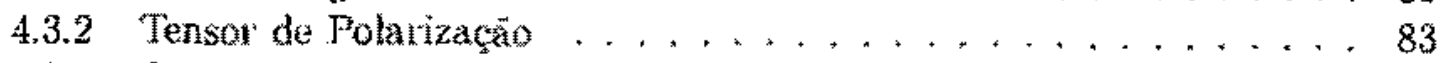

4.3 .3 Correcăo de Vértice e Alomento Magnético . . . . . . . . . . 84

4.3 .4 Espalhamento ................... 88

4.4 Análise ................................... 91

Conclusăo

A Tensor de Polarizacäo para MCS com Acoplamento Magnético 95

$\begin{array}{ll}\text { B Integrais } & 97\end{array}$

C Espalhamento em 1-Loop (Spin 0) 100

D Espalhamento em 1-Loop (Spin 1/2) 103

E Câlculos em 1-Loop (Spin 1) 107

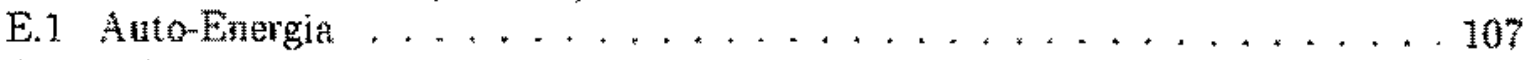

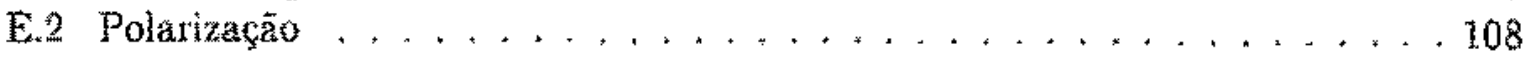

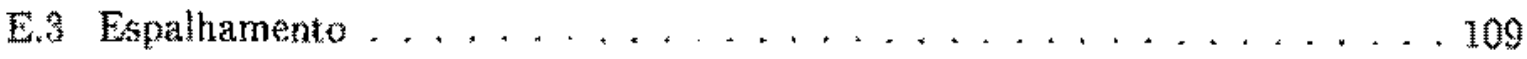

$\begin{array}{lr}\text { Bibliografia } & 112\end{array}$ 


\section{Introdução}

Teorias com o termo de Chem-Simons tem constituido objeto de interesse a partir de 1980, devido a importantes propriedades teóricas[1] e possiveis àplicaçōes em sisternas cujo comportamento principal se reduz a interaçoes num plano. Por exenplo, essas teorias tem sido úteis na explicaçăo do efeito Hall fracionário[?] e, possivelmente, no entendimento da supercondutividade a alta temperatura [3].

Entre as várias características que surgen en teorias em $(2+1) D$ com a presença do termo de Chern-Sinons (CS), ressaltan-se certos aspectos inusitados, tais como a existência de campos de gauge massivos e a possibilidade de spin fracionărio. A presença do termo de Chern-Simons, adicionado a Lagrangeana do campo eletromagnètico, gera uma massa topológica para o campo vetorial, sen quebrar a invanância de gauge[1]. A existência desta massa pode ser verificada explicitamente pela presenţ̧a de um pólo en um valor năo nulo do momento no propagador do campo vetorial. Este fato é importante para eliminar os problentas de divergências infravermeltuas que aparecem na $Q E D \mathrm{em}$ $(2+1) D[4]$

A possibilidade de spin fracionário stá associadia ao fato de eque em $(2+1) D$ a álgebra de rotações é Abeliana, eliminando desta forma as restrições ao valor do spin. Assim a estatística quầntica ê definida por uma fase associada ao morimento de uma particula em relaçäo a outra[5]. A relaçäo entre partículas de spin fracionário (também conhecidas como anyons) e a presença do termo de Chern-Simons foi verificada primeiramente nas referências $[6,7]$. Os autores introduziram um campo de gauge f acoplado minimamente com a matéria, sendo que a dinâmica deste campo vetorial é dada pelo termo de ChernSimons. Deste modo o spin e a estatística são mudados pela irterarăa deste campo vetorial com a matériat. Neste contexto, partículau carregadas interagindo via potancial de ChernSimons tern associadas a si um ftuxo magnétito pontual. Assim, podemos simular o efeito Aharonov-Bohm[8] pela interaçāo de duas partículas via campo de Chern-Simons.

Nesta tese vamos estudar algumas propriedades de teorias contendo o termo de ChernSimons e outros campos com vários valores do spin. Mais explicitamente: no capitulo 1 , trataremos o problema da etuivalência entre o modelo de Naxwell-Chern-Simons (MCS) e o modelo Auto-Dual (AD). Cos capitulos 2 e 3 ramos trabalhar com campos nâo-Abelianos de spin 0 e $1 / 2$ interagindo com a campo de CS. No último capitulo analisaremos algumas propriedades de modelos para particulas carregadas de spin 1 interagindo com o campo 
de CS.

O modelo Auto-Dual dado pela Lagrangeana

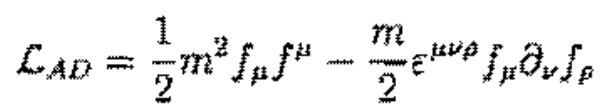

foi originalmente introduzido por Townsend et al.[9] como uma espécie de raíz quadrada do modelo de Proca, para descrever um único modo massivo, de spin $t=1$, propagando livremente. Foi mostrado, inicialmente por Jackiw e Deser[10] e posteriormente por vários autores $[1,12,13]$, que o modelo $\mathrm{AD}$ livre, ou mesmo com fontes externas, equivalente ao modelo de MCS descrito pela Lagrangeana

$$
L_{N C S}=-\frac{1}{4} F_{\mu \nu} F^{\mu w}+\frac{1}{4} m m^{k \mu \rho} A_{\mu} F_{\nu p} .
$$

Este fato foi usado para mostrar que no setor de baixa energia o modelo de Thirring massivo pode ser bosonizado[34]. Isto pode ster visto pela uso da equivalência do funcional gerador do Modelo de Thirring como o funcional gerador do modelo de MCS e a identificaçäo da conrente fermiônica com urna corrente bosônica.

A equivalência dos modelos AD e MCS livres está ben estabelecida. No entanto, não ê imediato que esta equivalência persista quando acoplamoi estes modelos (MCS e AD) a outros campos dinatmicos. Verificarenos explicitamente no capitulo 1 o que acontece no caso do acoplamento como o campo de Dirac [15]. Mostraremos que para que exista a equivalência entre os modelos de NCS e AD quando acoplados com férmions, são necessários acoplamentos diferentes e especficos. Por exemplo, como veremos, um modelo onde o campo $\mathrm{AD}$ é acoplado minimamente con o campo de Dirac: será equivalente ao modelo de MCS acoplado via uma interaçāo magnética com o campo fermiònico, mais um termo de auto-interaçāo típo Thirring.

Nos capitulos 2 e 3 exploraremos o fato que o efeito Aharonov-Bohm (AB) pode ser simulado, en teoria quäntica de campos em $(2+1) D$, por un sistema de partículas carregadas interagindo via termo de CS. $O$ eféto $A B$ tem se mostrado objeto de grande interesse na consolidaçẳo da mecänica quaantica e no esclarecimento đa relevância do papel do potencial vetor da eletrodinâmiea. A importância do potencial eletromagnético tem sido bastante estudada tanto no campo experinental quanto através da análise teórica via a mecânica quântica. Trabalhos de revisäo podem ser encontrados nos artigôs de Olariu e Popescu[16] e Ruijsenaars[17] ou no livro de Peshkin \& Tonomura[18]. Outros trabalhos tratando o problema via cálculo exato, usando a mecanica quântica, săo dados nas referências $[10,20,21]$.

O problema perturbativo pode ser tratado em teoria quântica de campos, como já foi dito, usando-se o fato do termo de $C S$ simular o potencial de $A B$. Foi verificado para a teoria năo relativistica que no caso de spin zero é necessário adicionar um termo de interaça de contato, $\left(\phi^{*} \phi\right)^{2}$, de modo a manter a teoria renormalizável e recuperar a invariância conforme[22]. Esta interação de contato aparece naturalmente no caso de férmions, via termo magnético de Pauli[23]. 
Vảrias pessoas trabaharam tentando entender a relaçâo entre o termo de contato na teoria de campos não relativística e certas classes de Hamiltonianos auto-adjuntos em mecảnica quântica[24, 25, 26]. Em particular, na referência [25], sob certas condiçōes e para um valor especifico da constante de acoplamento, os autores verificaram gue a divergencia é cancelada em todas as ordens da teoria de perturbaçăo e a invariância de escala é recuperada, além de desenvolveren uma conexão apropriada com a descrição de primeira quantizaçăo. No entanto, essa equivalêneia direta parece ser válida somente para algumas sub-familias de extensōes autondjuntas de Haniltonianos quânticos[26].

Partindo de um ponto de vista mais básico, podemos tratar o efeito $\mathrm{AB}$ a partir da teoria de campos relativística e entäo tomar o limite não relativistico apropriado. Procedendo desta maneira, efeitos das correçoes radiativas, como polarização do vácuo e momento magnético anờmalo, sâo incluidos automaticamente. No entanto esse limite em teoria quânitica de tampos năo é trivial. Um procedimento equivalente ao usado na redução da mecânica quântica relativistica, tal como Foldy-Wouthuysen[27], nắ é capaz de levar em conta todos os efeitos devido às correçoes radiativas[28]. Recentemente tem sido proposto um procedimento alternativo e sistemático pura obter esse limite en teoria quântica de campos $[23,29,30]$. Deste modo obten-se o resultado da teoria năo relativística, além dos releridos efeitos vindos das correyoues radiativas.

O procedimento citado acima consiste em calcular a amplitude de espalhamento relativistica, e, posteriomente, tomar o limite para pequenos momentos externos $(\mathbf{p}<<m)$. Os momentos internos (integraçôes nos loops) são separados em duas regiōes: a primeira contendo os estados intermediários de baixa energia (L), correspondendo aos momentos internos no intervalo de $(0, A)$, onde $A$ é um corte auxiliar intermediărio e que seră identificado com o corte näo relativistico: a segunda regiäo, de altas energias (H) correspondendo ao intervalo de $\left(A, \lambda_{0}\right)$, onde $\Lambda_{0}$ é o corte relativistio $\left(\Lambda_{0} \rightarrow \infty\right)$. Assim podemos observar quais são os efeitos dos estados de altas energias sobre os estados de baixas energias. Para termos controle nas expansōes que serào efetuadas nas duas regiöes de integraçấo adotaremos as seguintes condiçōes

$$
|\mathrm{p}|<<A<<m \quad\left(\frac{|p|}{\mathrm{d}}\right)^{2} \approx\left(\frac{A}{m}\right)^{2} \approx \frac{|\mathrm{p}|}{m}=n .
$$

onde $\eta$ é o parâmetro de expansäo. Xa regiào de baixas energias: todos os momentos espaciais envolvidos são pequenos, e assim podemos efetuar uma expansäo em $1 / \mathrm{m}$. Na regiào de altas energias o integrando pode ser expandido em série de Taylor em torno de $\mathrm{p} \approx 0$.

Este procedimento quando aplicado a teoria abeliana tanto escalar quanto espinorial[23, 29,30], mostra que a contribuiçăo dominante de baixa energia reproduz o resultado obtido pela troria näo relativistica, com $A_{\mathrm{R} R}$ identificado com A. A contribuiçâa de alta energia funciona como contra-termo para a parte de baixa energia. Os termos sub-dominantes seriam correçōes esugerem novos termos de interaçāo na Lagrangeana näo relativistica, como acontece en teorias efetivas $[31]$. 
Os resultados do estudo do efeito $A B$ para os cases escalar e espinorial das referencias acima, mostraram que existem algumas particularidades devidas ao spin da particula/23, $29,30]$. Nos capítulos 2 e 3 vamos verificar qual é o efeito de adicionarmos um indice de simetria interna para o campo de matéria, ou seja, se existe alguma peculiaridade no caso do seito AB năom Abeliano.

$O$ efeito $\mathrm{AB}$ näo-Abeliano[32, 33, 34, 35] tem se mostrado interessante, tanto no aspecto da investigaçäo teórica, cono em possiveis aplicaçôes em física de cordas, buracos negros e na gravitaçäo em $(2+1)$ dimensōes[36, 37, 38, 39, 40,41]. De acordo com ideias modernas, num estágio inicial da evolução, o universo era caracterizado por altas temperaturas e grau de simetria maior que agora. Com o resfriamento haverja uma série de transiçoes de fase e quebras de simetria. Como resultado foram formados defeitos topológicos (monopơlos, cordas e paredes de dominio) cuja existência tem importantes consequências em cosmologia[40]. Entre estes, as cordas contendo fluxos näo-Abelianos são objetos cosmológicos de especial importância e podem ter urn papel relevante nos centros de nucleaçăo de galáxias e lentes gravitacionais[41]. Neste sentido é interessante considerar situações onde há partículas espalhadas por um fluxo de tampo magnético năto-Abeliano.

No ultimo capítulo vamos estudar o problema da particula carregada de spin I interagindo com o campo de CS. Um aspecto interessante decorre do fato que em $(2+1) D$ temos mais do que uma formulação para descrever uma particula massiva de spin 1. Mesmo que aparentemente essas formulaçōes tenham alguma relação, qquando acoplamos estes modelos com o campo de CS começam a surgir as diferenças. No caso dos modelos de Proca e MCS, vemos que já a nivel de ărvore, para que a seçào de choque do dois modelos sejam equivalentes, näo podemos ter o mesmo tipo de acoplamento. Ou seja, se acoplamos o modelo de Proca minimamente com $\mathrm{CS}_{\text {, }}$ o acoplanento do modelo de MCS com o campo de CS não será minimal. A nixel de 1-loop o problema começa a ficar más complexo. Isto devido a estmutura dos propagadores dos modelos de Proca e MCS que apresentam diferenças significativas. Além disto, no caso especifico do modelo de MCS, os cálculos começam a se tornar invituveis devido ao grande numero de parâmetros de Feynman que surgem. Devido a isto, nós iremos implementar a correçāo de 1-loop, para os gráficos potencialnente divergentes, somente no caso do modelo de Proca acoplado minimamente com CS.

Finalizando, faremos alguns comentários sobre os resultados obtidos, e, apresentaremos alguns apêndices con o objetivo de dexar mais claro o procedimento usado no cálculo dos gráficos de Feynman. 


\section{Capítulo 1}

\section{Equivalência entre os Modelos Auto-Dual e Maxwell-Chern-Simons Acoplados com Férmions}

\subsection{Introdução}

A análise da equitralência de modelos aparentemente näo relacionados, tem se mostrado interessante principalmente na fisica em duas e trếs dimensöes. Exemplo disto em $2 \mathrm{D}$, é o estudo da equiralência entre o modelo de Thirring e o setor fermiónico do campo de Dirac interagindo via acoplamento derivativo com dois campos bosonicos[43]. Neste mesmo sentido, temos os trabalhos sobre bosonizaçāo, relacionando modelos fermiônicos com modelos bosônicos[44]].

En 3D, algumas tentativas de bosonizaçäo partem do fato que há duas maneiras para descrever um túnico modo massivo, de spin 1, se propagando livemente. De fato, nós temos o modelo Auto-Dual $(A D)$ live descrito por

$$
\mathcal{L}_{A D}=\frac{1}{2} m^{2} \int_{p} f^{\mu}-\frac{m}{2} e^{\# w_{B}} \int_{H z} \partial_{k} f_{p} .
$$

e o modelo de Maxwell-Chem-Simons (MCS) livre, dado pela densidade de Lagrangeana

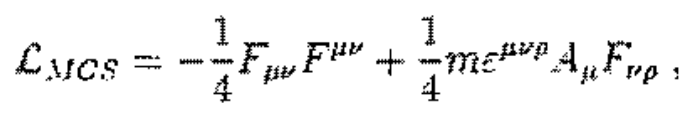

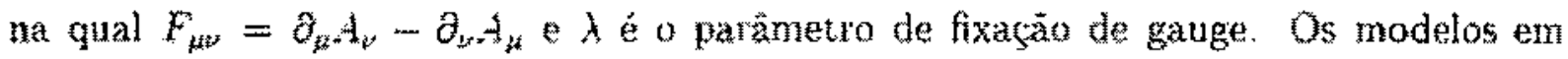
(1.1) e (1.2) aparentemente não ša equivalentes, visto que o primeiro näo é invariante de gauge enquanto o modelo dado em (1.2) é insariante de gauge. Apesar disto, ambos os modelos acima descretem um ünico modo massivo se spin 1 se propagando. O fato 
dos dois modelos terem o mesmo espectro massivo pode ser visto direto dos pólos dos propagadores. Realmente, de $\{1.1)$ e $(1,2)$ obtemos as propagadores

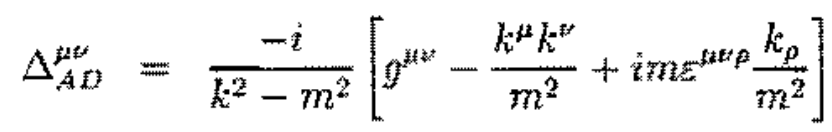

para o campo $f_{\mu} \mathrm{e}$

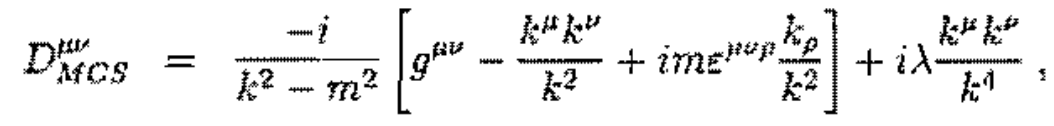

para o campo $A_{j}$, mostrando que ambos tem 0 mesmo pólo an $k^{2}=m^{2}$, Deser e Jackiwinol mostraram que na realidade ambos os modelos säo exuivalentes e estão rela* cionadoa através da identificatên

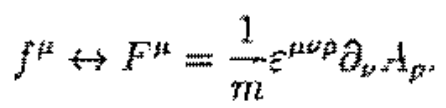

Isto pote ser visto tanto via análise direta das equaçōes de novimento para $f_{\mu}$ e $A_{\mu}$, du através do uso da Lagrangeana "master" on interpolante

$$
E_{y}=\frac{m^{2}}{2} f_{H} f^{\mu}-m^{2} f_{\mu} F^{j}+\frac{1}{2} F^{\mu} A_{\mu}
$$

a qual evidencia que os modelos tem uma origen comum. Raalmente, da variação de $C_{\text {: }}$ em relaça a $f_{\text {ix }}$ temos que $f^{*}=F^{\mu}$, e usando isto para elminar $f_{\mu}$ de (1.6) obtemos

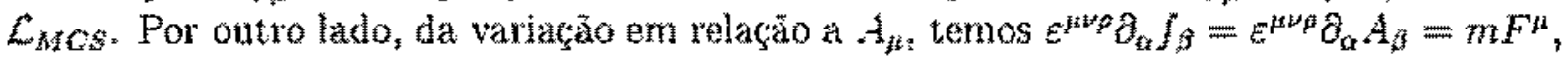

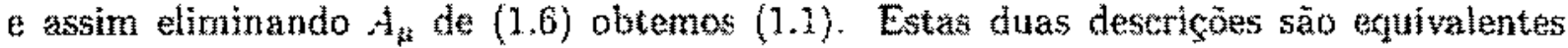
mesmo quando temos acoplamentos com fontes externas[10]. Estudos adicionais sobre a conexäo destes modelos[11, 12, 13,66] tem mostrado que o modelo Auto-Dual é uma versäo com gauge fxado do modelo de Maxwall-Chem-Simons.

A relacão mencionada antre os modelos $A D$ a $M C S$ tem sido usada para mostrar que no setor de baixa energiz o modelo de Thirring massivo pode ser bosonizado[14]. Mais precisamente, introduzindo un campo auxiliar $\left(f_{\mu}\right)$, o funcional gerador do modelo de Thirring pode ser escrito como

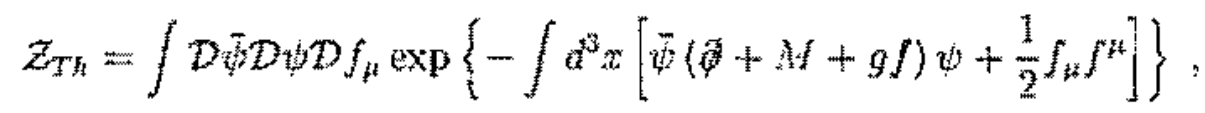

na qual $g^{2}$ é a constante de acoplamento do motelo de Thining . Efetuando a integraça nos campos fermionhicos e mantendo termos na ordem dominante na expansão em $1 / \mathrm{A}$,

\footnotetext{
iPara vermos que este funcional getador e equivalente ao funcional gerador do modelo de Thirring, basta integramos a equaça (1.7) sobre o campo ausiliar $f_{k *}$
} 
com a massa do fémion $M$ grande, podemos relacionar o funcional gerador do modelo de Thirring com o funcional gerador do modelo $\mathrm{AD}$, isto é

$$
Z_{T h} \approx Z_{A D}=\int \operatorname{Df} \exp \left\{-\int d^{3} x \mathcal{L}_{A D}\right\}
$$

Por outro lado, usando a equivalência entre os modelos $A D$ e $M C S$, temos

$$
\mathcal{Z}_{T h} \approx Z_{3}
$$

A extensăo deste resultado para o caso năo-Abeliano tem sido considerada por vários autores $[46,47,48,49,50]$.

A equivalência entre $0 s$ modelos $A D$ e $M C S$ estă bem estabelecida para o caso de campos livres. Neste caso, o campo básico do modelo $A D, f_{\mu}$, é visto ser substituído pelo rotacional do campo básico do modelo de MCS, A $A_{3 i}$. Resta entender o que acontece quando acoplamos estes modelos (MCS e $A D$ ) a outros campos dinâmicos. Aqui vamos especificamente estudar a equivatência entre os modelos $A D$ e MCS quando sta acoplados com férmions[1 15$]$. Por uma analogia com a equivalènéla no caso livre, quando acoplamos 0 modelo $A D$ minimarnente com os férmions $\left(f_{t} J^{k}\right.$, onde $J^{\prime \prime}$ é a corrente fermiônica), este modelo aparenta ser equivalente ao modelo de MCS acoplado com uma interação magnética, ou seja, ao rotacional da corrente fermionica. Esta tuquivalència aparente ê vista nas equaçöes de movimento para os campos $A D$ e MCS. Contudo, cono veremos na seçăo seguinte, contrariamente ao que acontece no caso de fontes externas; a equiralência dássica das correspondentes equaçōes do campo fermiônico, requer a inclusäo de uma interação adicional tipo Thirring.

\subsection{Identificação Clássica}

Vamos iniciar nossa análise considerando o tampo de Dirac acoplado minimamente con o campo vetorial do modelo $A D$. tal que a densidade de Lagrangeana vern a ser

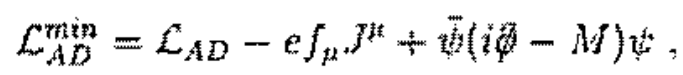

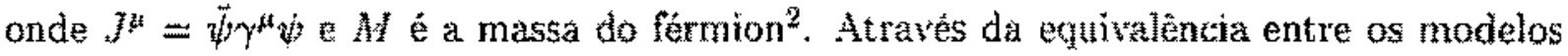
$A D$ e MCS, notamos que a simples identificacão $f^{\prime \prime} \rightarrow F^{\mu}$; a menos de uma derivada total, muda o acoplamento minimal para um acoplamento nagnético, isto é,

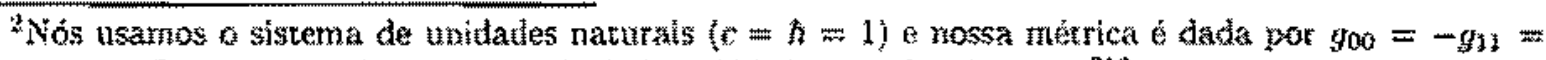

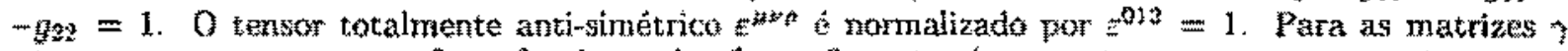

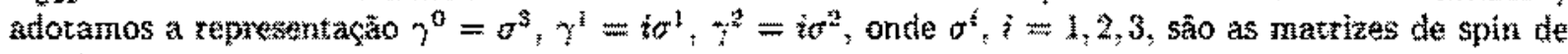
Patul.
} 


$$
f_{\not z} J^{\mu} \rightarrow A_{\mu q} C^{3 i}
$$

sendo $G^{H}=\frac{1}{n} e^{a s} \partial_{\alpha} J_{3}$. Isto sugere a equivalência do modelo (1.10) com o modelo descrito pela densidade de Lagrangeana

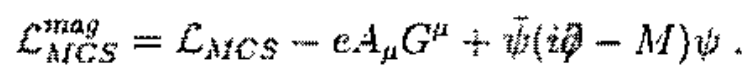

De fato, uma inspeção direta nas equações de movimento para o campo $f^{\mu}$ e $A^{\mu}$ levam a

$$
\begin{aligned}
& -m \varepsilon^{\mu \alpha \beta} \partial_{\mathrm{a}} f_{\beta}+m^{2} f^{\mathrm{t}}=e J^{\nu}, \\
& -m \varepsilon^{2 \alpha a t} \partial_{a} F_{g}+m^{2} F^{*}=e G^{\nu} \text {, }
\end{aligned}
$$

mostrando que o dual do campo de MCS satisfaz uma equação similar a equação obedecida pelo cempo AD mas com a correnté mudada pelo seu rotacional, isto é.

$$
f^{\prime \prime} \leftrightarrow F^{i} \Longrightarrow J^{\prime \prime} \leftrightarrow G^{z^{7}} .
$$

No entanto, para que a equivalência dos nodelos seja completa, é também necessário uma análise dos setores fermionicos. lamos usar as equaçós (1.13) e (1.14) para demonstrar que o setor fermiônico dos dois modelos serão iguais somente ste uma auto interação tipo Thirring com um constante de acoplamento especifica for adicionada a Lagrangeana (1.10).

Para vernos isto, consideremos a equaçăo de movimento para o campo fermiónico. Temos no caso do modelo $A D$

$$
(\hat{z})-M) \psi=e f_{H A} f^{*} \psi
$$

e para o modelo de $M C S$

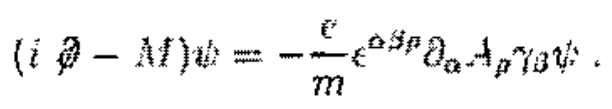

Através de (1.14) nós podemos expressar o campo de gatge $A_{4}$ em termos da fonte $G^{i t}$. Fealmente, $(1.14)$ pode ser escrita como

$$
\Gamma^{2 H} A_{14}=e G^{t y}
$$

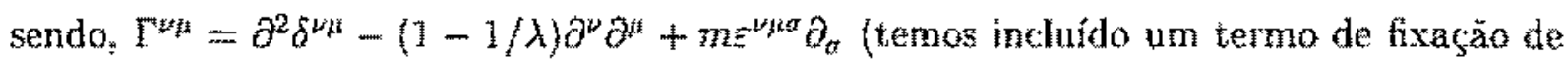

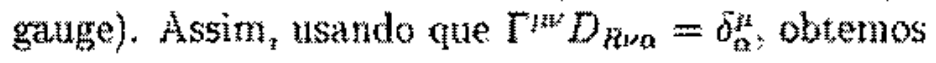

$$
A_{0}(x)=e \int d y D_{R \alpha \beta \mu}(x-y) Q^{\mu}(y),
$$


onde $D_{R a x a}$ e o propagador retardado para o campo de $M C S$, que no espaço dos momentos é dado por

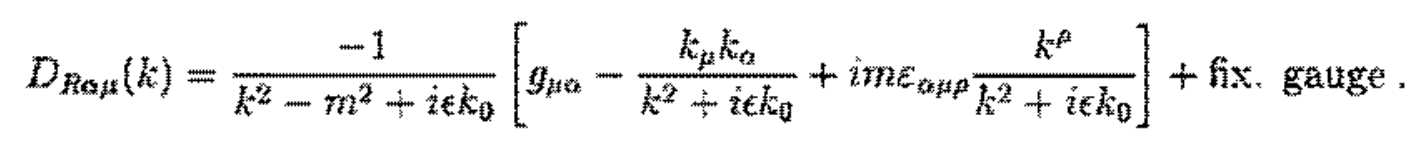

Substituindo (1.19) em (1.17) obtemos ${ }^{2}$

$$
(i)-M) \psi=e f_{\mu} \gamma^{\beta} \psi-\frac{e^{2}}{m^{2}} y^{\beta} \gamma_{\beta} \psi
$$

na qual

$$
f_{p}(x)=e \int d y \Delta_{k i \alpha}(x-y) I^{\alpha}(y)
$$

e

$$
\Delta_{k \mu \alpha}(k)=\frac{-1}{k^{2}-m^{2}+i k k_{\alpha}}\left[g_{\mu \mathrm{s}}-\frac{k_{\mu} k_{\alpha}}{m^{2}}+i m \varepsilon_{\mu \alpha \rho} \frac{k^{p}}{m^{2}}\right]
$$

é o propagador retardado para o campo $A D^{4}$. Basicamente este resultado é devido a identidade algébrica

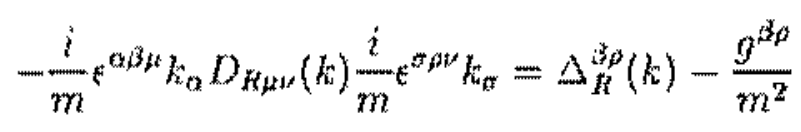

relacionando os propagadores do modelo AD e MCS.

Comparando as equaçoes (1.16) e (1.21) vemos que o setor fermiônico dos dois modelos são equivalentes somente se incluirmos em un dos modelos a interaç̧̌̉o tipo Thirring. Ou seja, o modelo

$$
\mathcal{L}_{A D}-e f_{\mu} J^{\mu}+\bar{\psi}(i \theta-M) \psi+\frac{e^{2}}{2 m^{2}} J^{\mu} J_{\mu}
$$

e não (1.10) ten o mesmo setor fermiônico que (1.12). Lsando (1.19), (1.20) e a definição de $F^{\mu}$ é fácil verificar que podemos escrever

$$
F^{n}=f^{\prime}-\frac{e^{2}}{m^{2}} n^{n}
$$

Através desta relaça podemos ver que (1.14) vai em (1.13). A partir do que foi visto, é claro que a interacäo tipo Thirring pode ser incorporada ao modelo $A D$ como feito acima ou ao modelo de MCS como veremos na seça seguinte.

\footnotetext{
3O termos de fixaçầ de gauge năo dará nentuma contribulção.

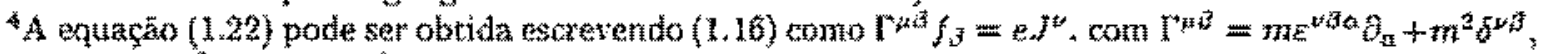

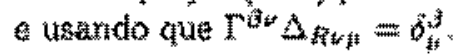




\subsection{Identificação Quântica}

Nós queremos investigar se esta equivalência continua válida a nivel quântico. Aqui nós ssperamos alguns problemas pois o sistema descrito por (1.10) é renormalizável perturbativamente $e^{5}$, enquanto a Lagrangeana (1.12) é näo-renormalizével. Isto pode ser visto direto pela contagem de potência. $A$ dimensäo em unidades de massa de $A_{a}$ e $\dot{\psi}$ são $1 / 2$ a 1, respectivamente. Assim a dimensäo da interaçăo magnética e de Thirring săo iguais a $7 / 2$ e 4 , respectivamente, estragando a renormalizabilidade dos modelos ${ }^{6}$. Contudo esta dificuldade pode ser eventualmente contornada. Por exemplo, se tomarmos o caso de $N$ férmions a teoria passa a ser renornalizárel via expansäo $1 / N$ e neste contexto os modelos săo renormalizáveis.

Para uma fundamentaçăo mais sólida, nós iremos usar o funcional gerador

$$
Z(\psi)=\int D f^{\prime \prime} D A^{\prime \prime} \exp \left[i \int L_{l}\left(f, A_{2}, b\right)\right]
$$

com uma Lagrangeana interpolante que generaliza $(1.10)$ e (1.12),

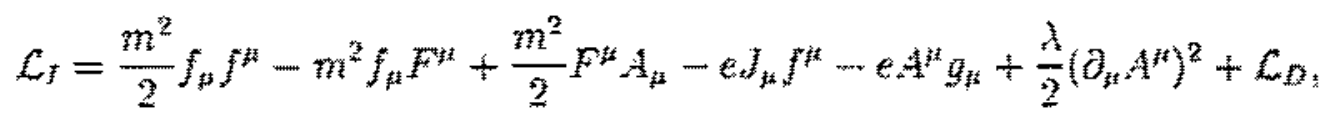

na çual $\mathcal{L}_{D}$ é a Lagrangeana de Dirac live e $g_{i k} . J_{j}$ säo correntes dependendo somente

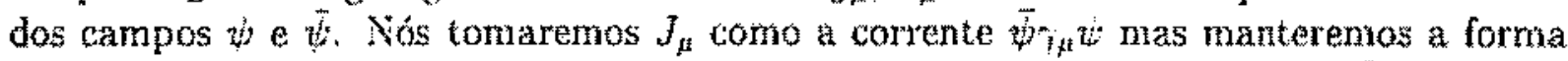
de $g_{\mu}$ arbitrária, exigindo, no entanto, que năo dependa dos campos vetoriais ${ }^{7}$.

Integrando sobre a configuração do campo $f_{\mu}$ tenıos

$$
Z(\psi)=\int D A^{u} \exp \left[i \int \mathcal{L}_{\mathrm{e} I J}^{(1)}(A, w)\right]
$$

onde

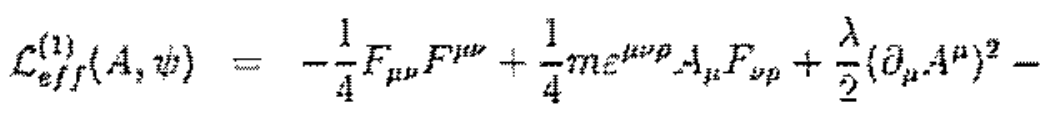

$$
\begin{aligned}
& e A^{\mu}\left(g_{\mu}+C_{\mu}\right)-\frac{e^{2}}{2 m^{2}} \cdot y^{\mu} J_{\mu}+\mathcal{L}_{D} \text {. }
\end{aligned}
$$

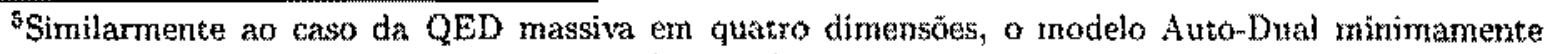
acoplado com os férmions te renormalizável. A parte longitudinal do propagador do campo vetorial tem urn comportameato tuim no ultravioteta, nâo se antiando para grandes momentos. Contudo, como este

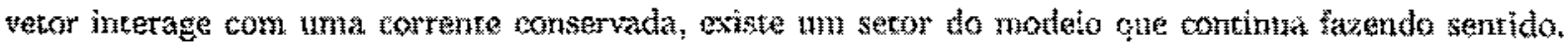

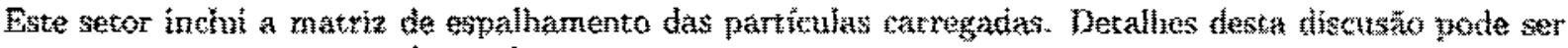
encontrado nas referencias [51, 32 ]

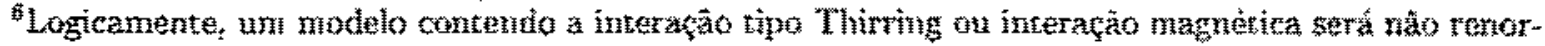

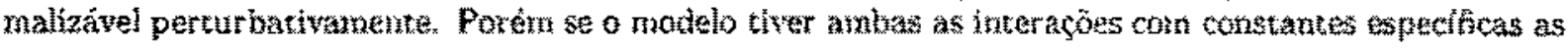
contribuiçōes poden ser somadas te nodo a tomar a modelo renomalizátel.

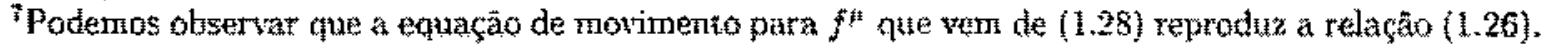


Por outro lado, integrando (1.28) sobre $A_{\mu}$ obtemos

$$
Z(\psi)=\int D f^{n} \exp \left[i \int \mathcal{L}_{e f f}^{(2)}(f, \psi)\right]
$$

sendo

$$
\begin{aligned}
\mathcal{L}_{e f f}^{(2)}(f, \psi)= & \frac{m^{2}}{2} f^{\mu} f_{\mu}-\frac{m}{2} \bar{m}_{\alpha \beta \gamma} f^{\alpha} \partial^{j} f^{\gamma}-c f^{\alpha}\left[J_{\alpha}+\left(g_{\alpha \beta}-\frac{\partial_{\alpha} \partial_{\beta}}{\partial^{2}}\right) g^{g}\right]+ \\
& \frac{e^{2}}{2 m} g_{\mu}\left[\xi^{\mu \nu \nu} \frac{\partial_{3}}{\partial^{2}}+\frac{m}{\lambda} \frac{\partial^{\mu} \partial^{\nu}}{\left(\partial^{2}\right)^{2}}\right] g_{\mu}+\mathcal{L}_{D} .
\end{aligned}
$$

Agora nós podemos considerar algumas possibilidades:

1. $g_{\mu}=0$. Nesta situação, (1.30) e (1.32) provam a equivalência quântica dos setores fermiônicos dos modelos discutidos na seçăo anterior, isto é, as Lagrangeanas (1.30) e (1.32) ficam

$$
\begin{aligned}
& C_{e f f}^{(1)}(A, \psi)=-\frac{1}{4} F_{p t r} F^{\mu t+}+\frac{1}{4} m_{t}^{\mu \nu \rho} A_{\mu} F_{p \rho}+\frac{\lambda}{2}\left(\partial_{1} A^{\mu}\right)^{2}- \\
& e A^{k} G_{\mu}-\frac{e^{2}}{2 m^{2}} J^{m} J_{\mu}+L_{D} \text {. }
\end{aligned}
$$

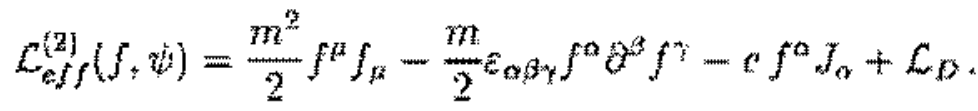

2. Se em vez disso, nós tomamos $g_{\mu}=-G_{\mu}$ entäo o campo de MCS desacopla e os fếrmions interagem atravẻs de uma interaçäo de Thirring, ou seja,

$$
\mathcal{L}_{e f f}^{(1)}(A, \phi)=\mathcal{L}_{M C S}+\mathcal{L}_{D}-\frac{e^{2}}{2 m^{2}} J^{\prime \prime} y_{n}+\frac{\lambda}{2}\left(\partial_{n} A^{\prime \prime}\right)^{2}
$$

Este modelo de campos fermionicos e vetoriais năo-interagentes é equivalente a um modelo que, alérn de uma auto-interaçăo, os fếrmions săo acoplados a um campo Auto-Dual. Este último modelo é descrito pela Lagrangeana

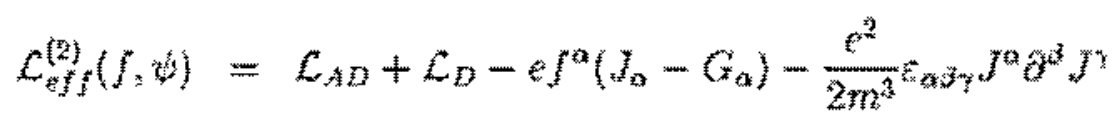

3. Como outra possibilidade tomenos $y^{n}=-y^{n}$. Keste caso (1.32) fica

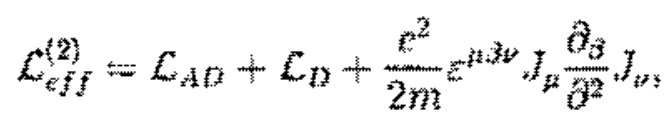


descrevendo campos Auto-Dual livres e férmions auto-interagentes. Por (1.30) este modelo é equivalente a um com férmions e campos vetoriais interagindo como especificado pela Lagrangeana efetiva

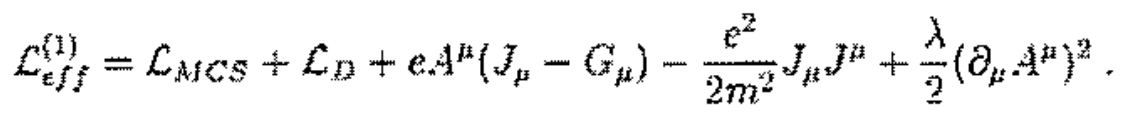

A generalizaçằ da equivalência dos modelos MCS e AD interagindo con outros campos dinầnicos, como por exemplo o campo escalar, nâo é trivial. De fato, neste caso a corrente depende explicitamente do campo de gauge $\mathrm{e}_{3} \mathrm{com}$ isso, o determinante funcional tem uma dependẻncia não-trivial com os campos de matéria.

Os modelos considerados envolvem acoplamentos não-renornalizaveis perturbativamente, como a interaçăo de Thirring e a magnética. Contudo, se os campos de férmions săo campos de $N$ componentes, os thodelos são renormalizáveis na expansäo $1 / N$. Para o modelo de Thirring isto foi provado em [52]. Aqui nôs examinaremos o caso quando o campo de MCS interage através de un acoplamento magnético, $A_{\mu} G^{\prime \prime}$. $O$ propagador $1 / N$ é dado pela soma da série

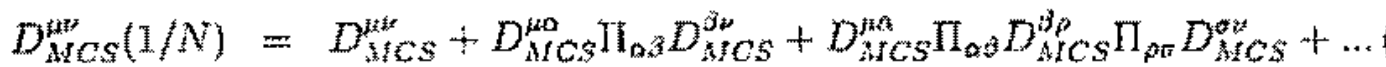

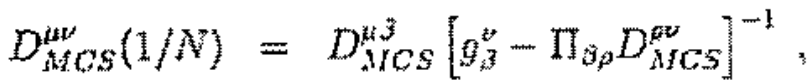

com $\prod^{\mu k z}$ sendo o tensor de polarização ${ }^{z}$

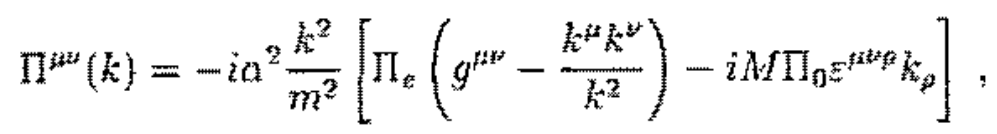

no qual $\alpha=\sqrt{N} e, \Pi_{0}$ e $\Pi_{\varepsilon}$ săo dados por

$$
\begin{aligned}
\Pi_{k} & =\frac{1}{4 \pi} \int_{0}^{1} d x \frac{2 h^{2} x(1-x)}{\left[M^{2}-k^{2} x(1-x)\right]^{1 / 2}} \\
\Pi_{0} & =\frac{1}{4 \pi} \int_{0}^{1} d x \frac{1}{\left[M^{2}-k^{2} x(1-x)\right]^{1 / 2}} .
\end{aligned}
$$

Lsando a expressào para $\Pi^{\text {tar }}$ e $D_{M C S}^{u t}$ podemos estrever o propagador completo na forma

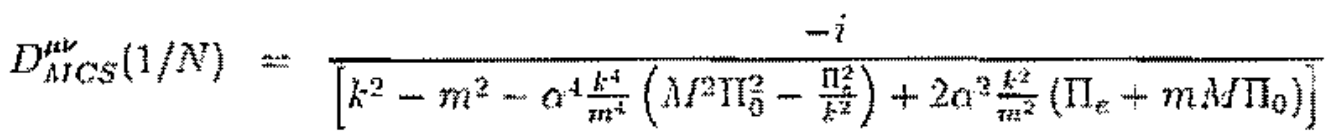

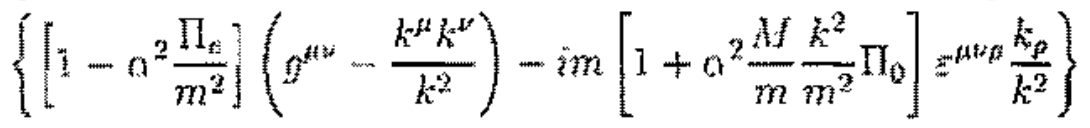

\footnotetext{
STeja apêridice $\mathrm{A}$
} 
Para $k \rightarrow \infty$, temos

$$
\Pi_{k} \rightarrow \sqrt{k^{2}} \quad \Pi_{0} \rightarrow \frac{1}{\sqrt{k^{2}}} .
$$

Assim, é fácil terificar que o propagador para o campo vetorial tem un comportamento com o momento $k$, tendendo para o infinito, como $1 /\left(k^{2}\right)^{3 / 2}$. Isso nos leva, pelo critério de contagem de potência: a conelusăo que o grau de divergêneia superficial de um gráfico genérico $G$ é

$$
d(G)=3-N_{F},
$$

sendo $N_{F}$ é número de linhàs externas de férmions em $G$. Aparentemente, aqui nós nos defrontamos com uma dificuldade visto que $d(G)$ não decresce com o número de linhas externas do campo vetorial crescendo. Contudo, (1.44) não leva em conta que, para um linha externa do vetor, a fator de momento associado com o vértice em que a linha é ligada não depende da variável de integraçäo ${ }^{3}$, tal que o grau de divergência efetivo é abaixado para

$$
d(C)=3-N_{F}-N_{B}
$$

que, como esperado, é o mesmo que no modelo de Thirring. Neste caso. é fácil de ver que os contra-termos necessảtios para fazer finita a funçäo de Green, tem a mesma forma dos termos já presentes na Lagrangeana original. Isto significa qute a teonia é renormalizável.

Por completeza, podemos verificar explicitamente a equivalëncia descrita no item 1 , isto $e_{1}$ con $g^{H}=0$, calculando a matriz $\mathcal{M}$ correspondente ao espalhamento de dois férmions. Para as Lagrangeanas (1.33) e (1.34) os vërtices de interação são

$$
\begin{aligned}
& \Gamma_{A D}^{\mu k}=-i e \gamma^{\mu} \\
& \Gamma_{M C S}=-\frac{e}{m} \gamma_{B} e^{b \alpha i s} k_{\alpha} \\
& \Gamma_{T h}=\frac{i e^{2}}{m^{2}} \gamma^{f^{z}} \gamma_{\eta_{k}},
\end{aligned}
$$

onde $k=p^{t}-p$, é omomento transferido. Os propagadores para os campos vetoriais $f_{p}$ e $A_{\xi 4}$ são os mestuos dados por (1.3) e (1.4). Para o propagador do férmion temos

$$
S(p)=\frac{i}{p-M}
$$

A nivel de árore, a matria espalhamento $M$ para o nodelo descrito por (1.33) 6

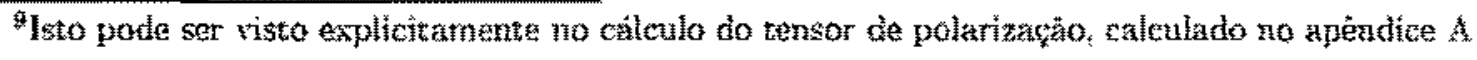




$$
\begin{aligned}
& M_{M C S-T \hbar}=\bar{u}\left(p^{\prime}\right) \Gamma_{M C s^{u}}^{\mu}(p) D_{\mu \nu}^{M C S}(k) \bar{u}\left(q^{\prime}\right) \Gamma_{M C s^{u}}^{u}(q) \\
& +u\left(p^{\prime}\right)\left(-i e^{*}\right) u(p)\left[\frac{i}{m^{2}} g_{z \nu}\right] \bar{u}\left(q^{*}\right)\left(-i e^{*}\right) u(q)
\end{aligned}
$$

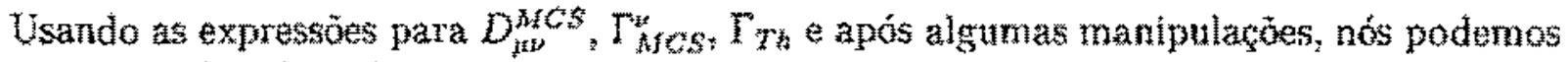
reescrever (1.50) na forma

$$
\mathcal{M}_{M C S-T h}=\vec{u}\left(p^{\prime}\right)\left(-i e \gamma^{\mu}\right) u(p)\left\{\frac{-i}{k^{2}-m^{2}}\left[g^{\mu \nu}-\frac{k^{\mu} k^{\nu}}{m^{2}}+i m \xi^{\mu \nu \rho} \frac{k_{\rho}}{m^{2}}\right]\right\} \tilde{u}\left(q^{\prime}\right)\left(-i e \gamma^{\nu}\right) u(q)
$$

isto é

$$
M_{M C S-T B}=M_{A D}
$$

Podemos facilmente verificar que a contraf̧ão de dois vértices do acoplamento magnético com o propagador de MCS, leva a um termo que é equivalente a contraçāo de dois vértices minimais con o propagador de $A D$, mais um termo que cancela a contribuiçăo do vértice de Thirring. Devido a isto, é fácil observar que a equivalència pode ser demonstrada ordem-a-ordern para a matriz espalhamento dos férmions. 


\section{Capítulo 2}

\section{Espalhamento Aharonov-Bohm Não-Abeliano para Partícula Escalar}

\subsection{Introduçāo}

O efeito AB nāo- 1 beliano tem sido analisado por vários autores[32,33,34,35] e tem se mostrado interessante tanto no estudo de gravitaga em $(2+1)$ dimensoes como na fisich de cordas cósmicas e buracos negros $\{30,37,38,30,40,41]$. No caso especial de cordas cósmicas, temoś associado a cada corda um fluxo magnético, de modo que o espalhamento de particulas carregadas por cordas, ou o proprio espalhanento entre cordas, pode ser tratado como um espalhamento $\mathrm{AB}$.

$O$ estudo do efeito $A B$ citado nas refereñucias acima foi feito através do cálculo exato via mecânica quaăntica. Como conhecido, no tratamento perturbativo da mecânica quântica para o caso A beliano, a aproximaçāo de Born falha em reproduzir a expansão do resultado exato[42], visto que o segundo termo da série de Born é infinito. Esta discrepância se deve ao fato que a auto-funçāo perturbativa năo satisfazer as mesmas condiçoes de contorno do que no caso exato. Por outro lado, no tratamento perturbativo en teoria de campos não relativística para spin zaro theliano, foi mostrado que para eliminar as divergencias, restaurar a invariância de escala e reproduzir o resultado da expansăo da soluçāo exata, é necessário adicionar um termo de contato $\left\langle\omega^{*} \phi\right\}^{2}[22]$.

Recentemente o tratamento perturbativo foi aplicado para a teoria de campos nāo relativistica näo-Abeliana[6] ], isto é, partindo de um modelo para partículas escalares nào relativísticas, com um índice de simetria interna, acoplado minimamente com o campo de CS nāo-Abeliano, foi calculado o espathamento ate 1-loop. Neste casto os autores mostram que para manter a teoria finita e recuperar a invariancia de escala, e também para obter o resultado da expansăo do cálculo exato, te necescário a adiçào de uma interação quártica. como acontece no caso Abeliano.

Neste capitulo vamos estudar o efeito $A B$ năo-Abeliano de spin zero, como o limite 
da terria quántica de campos relativistica, usando o procedimento do corte intermediátio descrito na introdução. Com o objetivo de fixar a notação e facilitar a comparação com o resultado obtido da teoria relativistica, vamos considera brevemente o caso não ralativistico, usando a regularizaçăo por corte. Depois disto, vamos calcular o espalhamento AB a partir da teoria relativistica tomando o limite para pequenos momentos externos[62]. Veremos que este procedimento, alén de reproduzir o caso Abeliano, nos dá em ordem dominante o resultado obtido via călculo da teorizaño relativistica. Em ordem sub-dominante surgem correcoses que induzem novos termos na Lagrangeana efetya năo relativisticz. No apéndice $\mathrm{B}$ nós temos algumas ralaços a integraís útais no cálculo do

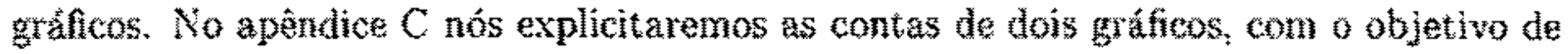
deixar claro procedimento apheado no cálculo das amplitudes de Furnman.

\subsection{Modelo Não Relativistico}

Inicialmente, vamos analisar o tratamento pertu bativo pas o modelo näo-Abeliano nằ relativistico. A Laglangeana nào relativistica clasticamente pode ser obtida da rela. tivistica por uma redefniçăo dos campos, extraindo-se a lator de fase associado a energia de repouso da particula, exp $\left(-i m c^{2} t / \hbar\right)$, expandindo en potencias de $1 / c$ (ou $1 / m$ ). Até ordem dominante e eliminando-se os termos vapidamente oseilantes 63 ] temos

$$
\begin{aligned}
\mathcal{L}_{N R 2} & =-\Theta^{a 3^{-}} t r\left(A_{\alpha} \partial_{B} A_{\gamma}+\frac{2 g}{3} A_{\alpha} A_{\beta} A_{n_{j}}\right)+i \Phi^{\dagger} D_{l} \Phi-\frac{1}{2 m}(D \Phi)^{\dagger}(D \Phi) \\
& -\frac{1}{4} \Phi_{n^{\prime}}^{\dagger} \Phi_{m^{\prime}}^{\dagger} C_{n^{\prime} m^{\prime} n m} \Phi_{m} \Phi_{m} .
\end{aligned}
$$

onde $\Phi$ é um campo complexo transformando-se com a representacăo fundamental do grupo de gauge $\mathrm{SU}(\mathrm{n})$, gerado pelas matrizes $T^{n}$, con $0=1,2, \ldots \ldots N=n^{2}-1$, e $A_{\mu}=A_{n}^{a} T_{0}$ e $D_{n}=\partial_{\mu}+9 \cdot 4_{\mu}$. As matrizes $T^{\sigma}$ satistazem a algebra de lio

$$
\left[T^{4}: T^{k}\right]=\int_{\Delta t e} T^{5}
$$

nomalizadas por

$$
\ln \left(T^{a}, T^{4}\right)=-\frac{1}{2} b_{b s}
$$

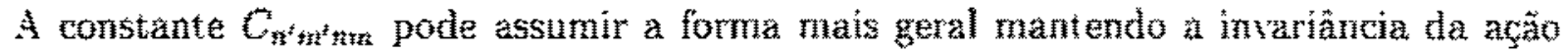
sobre as transtormaçoes do grupo $S E(n)$.

\footnotetext{
'Podemos tamben usar o procedimento le Foldy-Wouthysen. No entanto este procedinento e

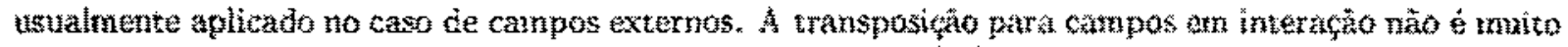

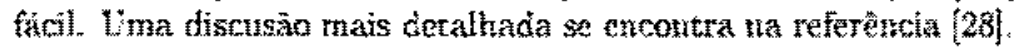


Para facilitar o cálculo da amplitude de spalhamento vamos abstrair os índices de matéria, trabalhando com uma matriz $\mathcal{M}(\theta)$ tal que a amplitude de espalhamento será dada por

$$
-i A=\left\langle n^{\prime}, m^{\prime}|\mathcal{M}(\theta)| n, m\right\rangle+\left\langle m^{t}, n^{*}|\mathcal{M}(\theta+\pi)| n, m\right\rangle,
$$

onde $\theta$ e ângulo de espalhamento. Assim,

$$
\begin{aligned}
& C_{n^{\prime} m^{t} t m}=\left\langle n^{\prime}, m^{\prime}|C| n, m\right\rangle \\
& \left(T^{\mathrm{a}}\right)_{\pi^{\prime} n}\left(T^{b}\right)_{m i}=\left\langle n^{\prime}, m^{l}\left|T^{a} \otimes T^{b}\right| n, m\right\rangle \\
& C_{n^{\prime} m^{t} n n_{m}}^{2}=\sum_{l_{y} t^{\prime}}\left\langle n^{t}, m^{\prime}|C| \ell^{\prime} l^{\prime}\right\rangle\left\langle l, l^{\prime}|C| n, m\right\rangle \text {. }
\end{aligned}
$$

Para obter as regras de Feynman vamos adicionar na Lagrangeana (2.1) o termo de fixação de gauge (gauge de Coulomb)

$$
\mathcal{L}_{F G}=-\frac{1}{\xi} \operatorname{tr}(\nabla \mathrm{A})^{2}
$$

e o termo de fantasma (ghost)

$$
E_{G H}=-\eta^{* \hbar}\left(\nabla^{2}+g \int_{a b c} \mathrm{~A}^{c}, \nabla\right) \eta^{2} .
$$

No limite $\xi \rightarrow 0$ as regras de Feyman, representadas graficamente na figura $(2.1)$, s薪安:

Propagador do campo de matéria

$D(p)_{n m}=D(p) \delta_{n m}=\frac{i}{p_{0}-\frac{\mathrm{p}^{2}}{2 m}+i \epsilon} \delta_{n m}$

Propagador para o Campo Fantasma

$G(p)_{b a}=G(p) \delta_{b a}=\frac{-i}{p^{2}} \delta_{b a}$

Propagador para o campo de gange

$D^{\mu \nu}(k)_{b a}=D^{m \nu}(k) \delta_{b a}=\frac{1}{\Theta} \varepsilon^{z b \nu \lambda} \frac{\bar{k}}{\mathbf{k}^{2}} \partial_{b a}$

Vértices de Interaçāo

$$
\begin{aligned}
& \Gamma_{\text {min }}^{a, 0}\left(p, p^{*}\right)=-g\left(T^{\tilde{a}}\right)_{m m} \\
& \Gamma_{n m}^{a, j}\left(p, p^{\prime}\right)=-\frac{g}{2 m}\left(T^{a}\right)_{m m}\left(p+p^{t}\right)^{i}
\end{aligned}
$$




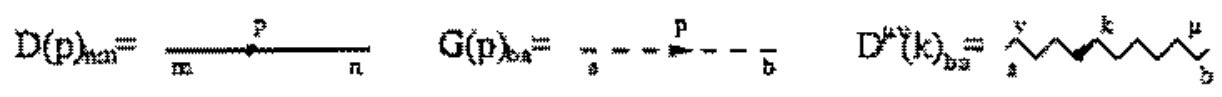
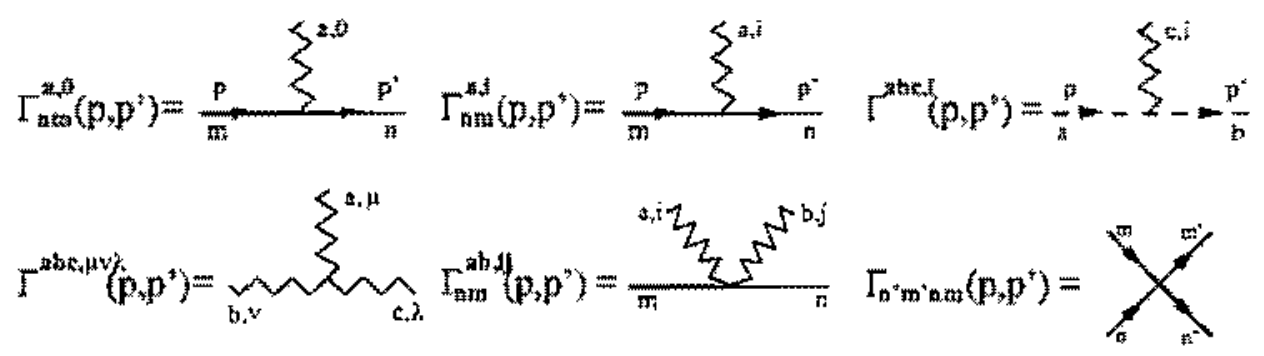

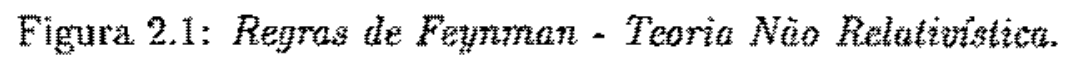

$$
\begin{aligned}
& \Gamma^{a b c, i}\left(p, p^{j}\right)=-g f^{a k k j} p^{y i}
\end{aligned}
$$

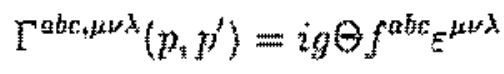

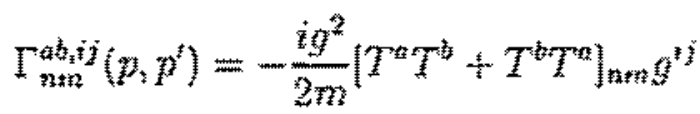

$$
\begin{aligned}
& \Gamma\left(p, p^{t}\right)_{m n^{n} n_{n n}}=\frac{-i}{2} C_{n^{i} n^{3} n n}
\end{aligned}
$$

Onde, no propagador do campo de gauge, $\tilde{h}_{\lambda}=\left(0, k_{1}, k_{2}\right)$.

Os gráficos que contribuem para o espalhamento na ordem de arvore são representados na figura $(2,2)$, e nos levam a amplitude de espahamento ${ }^{2}$

$$
M(\theta)=-\frac{C}{2}-i \frac{2 \pi}{m} n \cot (\theta / 2)
$$

onde estamos usando a mesma notaçăo da referencia [61]

$$
\Omega=\frac{-g^{2}}{2 \pi \Theta} T^{a} \otimes T_{\alpha}, \quad \zeta^{2}=\frac{9^{4}}{4 \pi^{2} \Theta^{2}} T^{a} T^{b} \otimes T_{t} T_{b}
$$

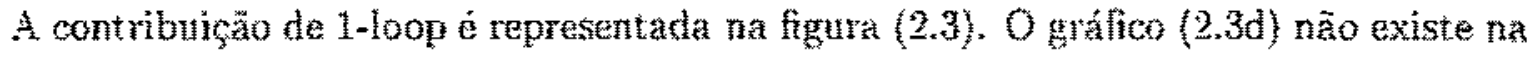
teoria Abeliana, vai ser essencial para cancelar as divergencias e reproduzir o resultato da expansào do caso axalo. 0 cálculo destes graficos nos leva $a^{3}$ (para $\theta 0$ )

\footnotetext{
${ }^{2}$ Todos os cáculos da amplitude de espathamento serino feitos no centro de massa.

${ }^{3}$ No apêndice $\mathrm{C}$ expiticitaremos as contas do gráfico $(2.3 \mathrm{~b})$.
} 


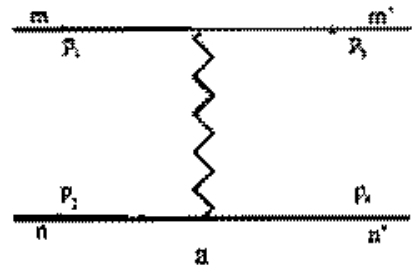

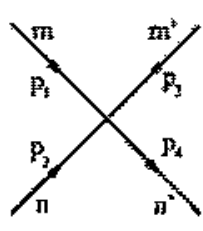

b

Figura 2.2: Espahamento a niwel de Arvore - Teoria Nöo Relativiatica,

$$
\begin{aligned}
& M_{\mathrm{a}}(\theta)=\frac{m C^{2}}{16 \pi}\left[\log \left(\frac{\mathrm{A}_{\mathrm{g}}^{2}}{\mathrm{p}^{2}}\right)+i n\right] \\
& \mathcal{M}_{\mathrm{b}}(\theta)=\frac{-\pi \Omega^{2}}{m}[2 \log |2 \sin (\theta / 2)|+i \pi] \\
& M_{c}(\theta)=\frac{-m \Omega^{2}}{m}\left[\log \left(\frac{1 \mathrm{~s} F}{\mathrm{p}^{2}}\right)-2 \log |2 \sin (\theta / 2)|\right]
\end{aligned}
$$

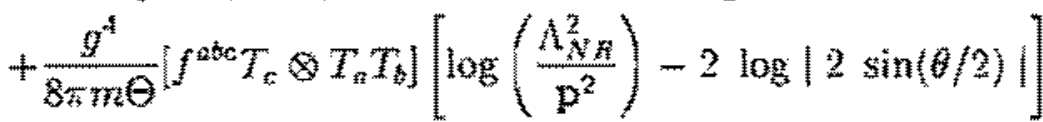

$$
\begin{aligned}
& M_{\mathrm{d}}(\theta)=\frac{-g^{4}}{8 \pi m \theta}\left[f^{n b c} T_{c} \otimes T_{\mathrm{a}} T \mid\left[\log \left(\frac{A_{\mathrm{N} N}^{2}}{\mathrm{p}^{2}}\right)-2 \log |2 \sin (\theta / 2)|+1\right] .\right.
\end{aligned}
$$

Aqui foi usado que $\left[T^{a}, T^{b}\right]=f^{a b c} T_{c}$ e $\int_{a b c} f_{a b d}=\delta_{c d}$. Observe que a contribuição de $\mathcal{M}_{\mathrm{d}}(\theta)$, a menos do termo constante, cancela a segunda parte de $\mathcal{M}_{\mathrm{c}}(\theta)$ que surge devido a estrutura do grupo não-Abeliano. Assirn temos que, a menos do terno constante en (2.22) que pode ser absonido en $C$.

$$
M_{1 \text { matap }}(\theta)=\frac{m}{16 \pi}\left(C^{2}-\frac{16 m^{2} n^{2}}{m^{2}}\right)\left[\log \left(\frac{A_{N B}^{2}}{p^{2}}\right)+i \pi\right]
$$

Observenos que para $C^{2}=16 \pi^{2} \Omega^{2} / m^{2}$ näo temos correção de L Loop para o espalhamento de $A B$ na teoria não relativística. O resultado da equação (2.23) é o mesmo da referéncia [61], só que os autores usaram a regularizacăo dimensional, enquanto que aqui foi usado a regularizaçäo por cortc.

\subsection{Modelo Relativístico}

O eteito $A B$ pode ser tratado a partir de um ponto mais fundamental, isto é, podemos tratar o problema através do cálculo da amplitude relativistica, usando o método de separação das regiỏes de alta e baixa energía, como descrito inicialmente. Isto nos 


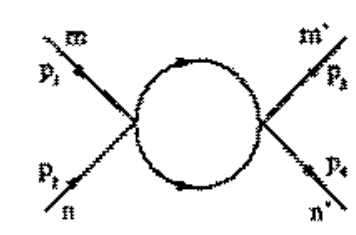

a

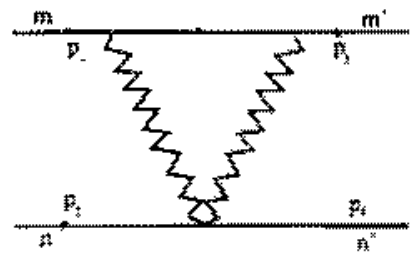

$\mathbf{c}$

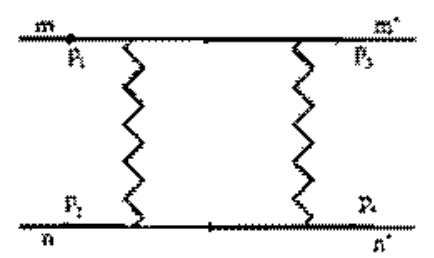

b

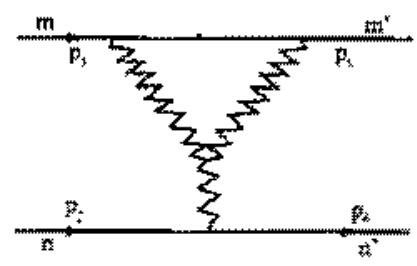

ส

Figura 23: Espalhamento 1-loop - Teoriu Näo Relativistica.

dará contribuiçōes advíndas das correços radiativas e que não aparecem na teoria näo relativística. No caso do modelo relativistico as amplitudes de Feynman ficam complicadas no caso de uma matriz geral $C_{n^{t} m^{x} n_{n}}$ do acoplemento quártico. $O$ problema torna-se razoavelmente mais simples no caso do grupos de simetria de gauge SU(2). Neste caso a Lagrangeana para o campo carregado de spin zero, minimanente acoplado com CS, e com a auto-interaçào mais geral que mantém a simetria do grupo, ẻ

$$
\begin{aligned}
\mathcal{L}= & -\Theta E^{\alpha \beta \gamma} t r\left(A_{\alpha} \partial_{b} A_{\gamma}+\frac{2 g}{3} A_{\alpha} A_{B} A_{\gamma}\right)+\left(D_{\mu} \Phi\right)^{\dagger}\left(D^{\mu} \Phi\right) \\
& -m^{2} \Phi^{\dagger} \Phi-\frac{A_{1}}{4}\left(\Phi^{\dagger} \Phi\right)^{2}-\frac{\lambda_{2}}{4}\left(\Phi^{\dagger} T^{\omega} \Phi\right)^{2}
\end{aligned}
$$

na qual $T^{n}$ são matrizes anti-hermilianas $2 \times 2, \operatorname{com} a=1,2,3$, satistazendo a álgebra de Lie

$$
\left[T_{a}, T_{b}\right]=-2 c_{j} \epsilon_{\text {alk }} T^{c}
$$

e normalizadas port

$$
\ln \left(T_{a} T_{b}\right)=-\frac{1}{2} \delta_{a b}
$$

Além disso temos outras relação para as matrizes $T^{\text {an }}$,

$$
\operatorname{tr}(I)=n \quad \operatorname{tr}\left(T^{2}\right)=0
$$

\footnotetext{
${ }^{4}$ Obs: Tentos que $c_{f}=-1 / 2, \delta_{\mathrm{a}}^{a}=3$ e $n=z$, porent vamos mantè-ias a fim de facilitar o limite para o caso Abeliano.
} 


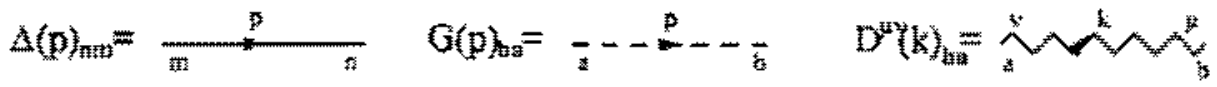

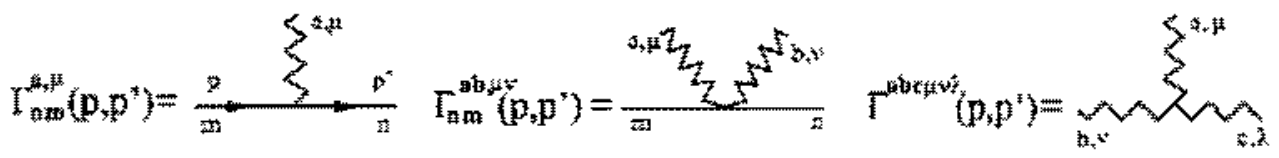

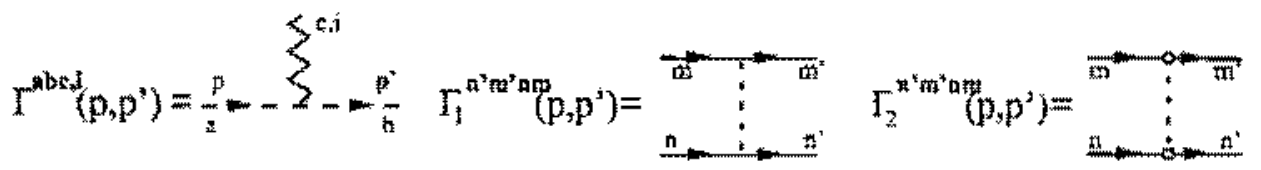

Figura 2.4: Regras de Feynman - Teoria Relativistica.

$$
\begin{aligned}
& \operatorname{tr}\left(T^{a} T^{b} T^{c}\right)=\frac{1}{2} c_{f} z^{a b b} \\
& T^{a} T^{b}=-\frac{1}{2 n} \delta^{a b} I-c_{j} z^{a b c} T_{c} \\
& \left\{T^{a}: T^{b}\right\}=-\frac{1}{n} \delta^{a b} \quad T^{a} T_{a}=-\frac{\delta_{a}^{a}}{3 n} I \\
& T^{a} T^{b} \otimes T_{a} T_{b}=\frac{1}{4 n^{2}} \delta_{a}^{a} I \otimes I+2 c_{f}^{2} T^{a} \otimes T_{a} \\
& T^{a} T^{b} \otimes T_{b} T_{a}=\frac{1}{4 n^{2}} \delta_{a}^{b} I \otimes I-2 c_{j}^{2} T^{a} \otimes T_{a}
\end{aligned}
$$

Novamente, para obtermos as regras de Feynman, vamos adicionar a Lutgrangeana (2.24) o termo de fixaçào de gauge, equação (2.0), e o termo do campo fantasma, equação (2.7). No limite $\xi \rightarrow 0$, obtemos as regras representadas na bura (2.4), e que analiticamente sẫo dadas por

$$
\begin{aligned}
& \text { Propagador do campo de matéria } \\
& \Delta(p)_{n m}=\Delta(p) \delta_{n n}=\frac{i}{p^{2}-m m^{2}+i t} \delta_{n m}
\end{aligned}
$$

O propagador do campo de gauge e do campo fantasma são os mesmos dado por (9) e (10). Para os vértices de interação temos ${ }^{5}$

\footnotetext{
${ }^{5}$ Os vértices $\Gamma_{1}$ e $\Gamma_{z}$ vern dos termos de interação $\frac{\lambda_{1}}{4}\left(\Phi^{\dagger} \Phi\right)^{2}$ e $\frac{\lambda_{2}}{4}\left(\Phi^{\dagger} T^{n} \Phi\right)^{2}$ respectivamente. A notação com a linha pontilhada e usada para facilitar a visualização do fluxo do campo de matéria. Ou seja, a linha pontilhacia funciona como um campo auxiliar cujo propagador é a identidade.
} 
Vêrtices de Interaçāo

$$
\begin{aligned}
& \Gamma_{n m}^{a, k}\left(p, p^{\prime}\right)=-g\left(T^{a}\right)_{n m}\left(p+p^{b}\right)^{n} \\
& \Gamma_{n=1}^{a b n t x}\left(p, p^{\prime}\right)=-i g^{2}\left[T^{a} T^{b}+T^{b} T^{a}\right]_{n n n} g^{a u t} \\
& \Gamma_{\searrow}^{n^{\prime} m^{i} n n}\left(p, p^{\prime}\right)=\frac{-i \lambda_{1}}{2} I^{i^{\prime} \pi} I^{m^{i} m} \\
& \Gamma_{2}^{n^{\prime} m^{\prime} n m}\left(p, p^{\prime}\right)=\frac{-i \lambda_{2}}{2}\left(T^{a}\right)^{n^{\prime} n}\left(T^{a}\right)^{m^{\prime} m} \\
& \Gamma^{a b c, i}\left(p, p^{\prime}\right)=-g \varepsilon^{a b c} p^{i}
\end{aligned}
$$

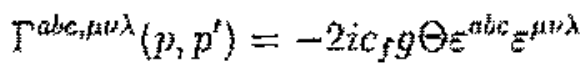

\subsubsection{Correção para a Auto-Energia}

Os graficos que aparecem na função de dois pontos para o campo de matéria, a nível de 1-loop, săo mostrados na figura (2.5). Os únicos grăficos diferentes de zero săo (2.3.a), $(2.5 . \mathrm{b}) \mathrm{e}(2.5 . \mathrm{c})$

$$
\begin{aligned}
& \Sigma_{a}^{n m}(p)=f^{n \pi m} \Sigma_{a}(p) \\
& \Sigma_{b}^{m m}(p)=\operatorname{tr}(I) \delta^{n m} \Sigma_{\mathrm{a}}(p)
\end{aligned}
$$

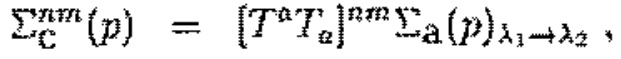

no qual

$$
\Sigma_{a}(p)=-i \frac{\lambda_{1}}{2} \int \frac{d^{3} k}{(2 \pi)^{3}} \Delta(k)=\frac{\lambda_{1}}{2} \int \frac{d^{3} k}{(2 \pi)^{3}} \frac{1}{k^{2}-m^{2}+i \epsilon} .
$$

Efetuando a integração em $k^{0}$ com o uso do teorema dos resíduos, temos que

$$
\Sigma_{\mathrm{a}}(p)=\frac{-i \lambda_{1}}{2} \int \frac{d^{2} \mathrm{k}}{(2 \pi)^{2}} \frac{1}{2 \omega_{k}}
$$


Agora separando na parte de baixa e alta energia e mantendo termos atê ordem $\eta^{2}$, temos que

$$
\begin{aligned}
& \Sigma_{3}^{L}(p)=\frac{-i \lambda_{1}}{16 \pi}\left[m\left(\frac{\Lambda^{2}}{2 m^{2}}-\frac{\Lambda^{4}}{4 m^{4}}\right)\right] \\
& \Sigma_{a}^{H}(p)=\frac{-i \lambda_{1}}{16 \pi}\left[2\left(\Lambda_{0}-m\right)-m\left(\frac{\Lambda^{2}}{2 m^{2}}-\frac{\Lambda^{4}}{4 \pi^{4}}\right)\right] .
\end{aligned}
$$

Observe que toda contribuição vem da parte de alta energia. A parte de baixa energia ê polinomial em $A$ a se cancela quando somado com a de alta energia. Se tivessemos usado a regularizaçào dimensional só teriamos a parte finita (nenhuma dependència em $\left.A_{0}\right)$. Como $\mathrm{E}$ não depende do momento externo năo teremos nenhuma renormalização para a funçăo de ondia até 1-loop. O termo acima pode ser absorvido numa renormalização de massa. Os demais gráficos se anulam pela anti-simetria do propagador de gauge ou por confugação de carga.

\subsubsection{Tensor de Polarização}

Os gráficos que surgem na funçăo de dois pontos para o campo de gauge estäo representados na figura (2.6). Observamos que para $\Pi_{\mathrm{C}}$ e $\Pi_{\mathrm{d}}$ só temos as componentes espaciais. Usando as regras de Feynman temos que[64]

$$
\prod_{\mathrm{C}}^{i j, a b}(q)+\prod_{\mathrm{d}}^{\mathrm{i} j, a b}(q)=\delta^{a b} C t e \int \frac{d^{2} \mathbf{k}}{(2 \pi)^{2}} \frac{k^{i}(k-q)^{j}-h^{j}(k-q)^{i}}{\mathbf{k}^{2}(\mathbf{k}-\mathbf{q})^{2}}=0
$$

Assim nós obtemos para ח ${ }^{\mu t}$

$$
\Pi^{\mu \nu, a b}(q)=\Pi_{\mathrm{a}}^{\mu \nu, a b}(q)+\Pi_{\mathrm{b}}^{\mu \nu, a b}(q)=\operatorname{tr}\left(\mathcal{T}^{a} \mathcal{T}^{b}\right) \Pi^{\mu \nu}(q),
$$

onde $\Pi^{\mu \prime}(q)$ é o mesmo obtido na teoria abeliana[29]

$$
\Pi^{\mu x}(q)=\frac{i g^{3}}{8 \pi} P^{j \mu^{\prime \prime}}(q) \Pi\left(q^{2}\right)
$$

com

$$
\begin{aligned}
& p^{\mu k}(q)=q^{2} g^{\beta *}-q^{a} q^{\mu} \\
& \Pi\left(q^{2}\right)=\int_{0}^{1} d x \frac{1-4 x+4 x^{2}}{\left[m^{2}-q^{2} x(1-x)\right]^{(1 / 2)}} \approx \frac{1}{3 m}\left[1+\frac{q^{2}}{20 m^{2}}\right] \text {. }
\end{aligned}
$$

Como no caso da teoria abeliana, a contribuição para o tensor de polarizaçăo vem somente da parte de altas energia. Este fato era esperado, pois esses grăficos năo existem na teoria näo relativistica. 

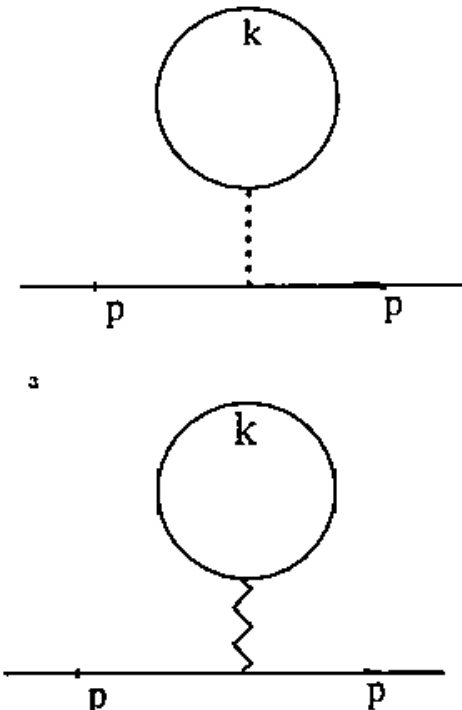

d

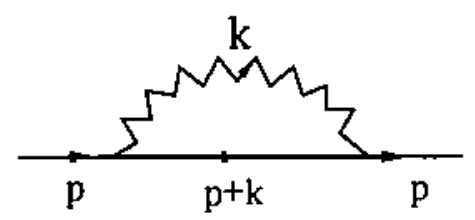

g

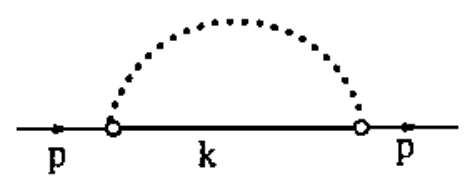

b

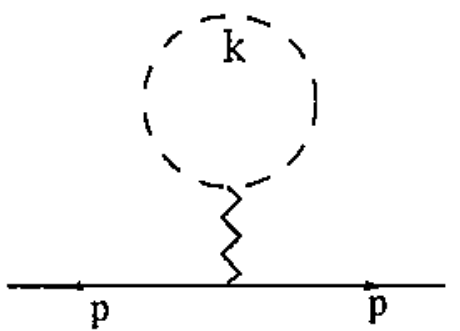

e

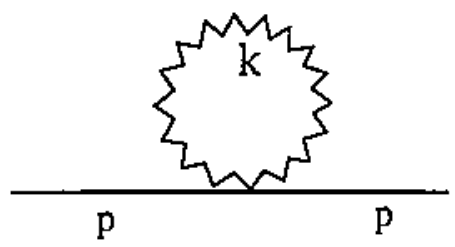

h

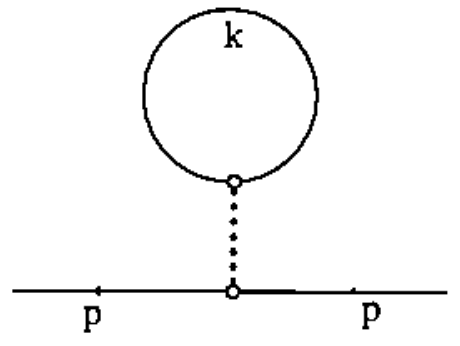

$c$

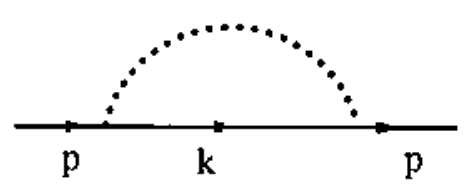

f

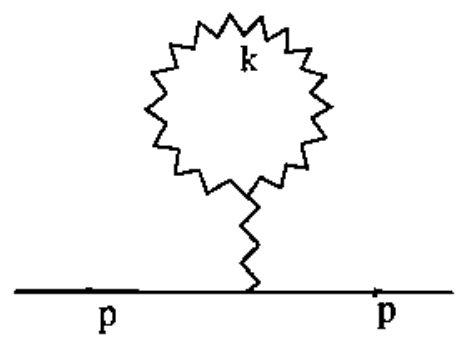

Figura 2.5: Auto-Energia - Teoria Relativística.
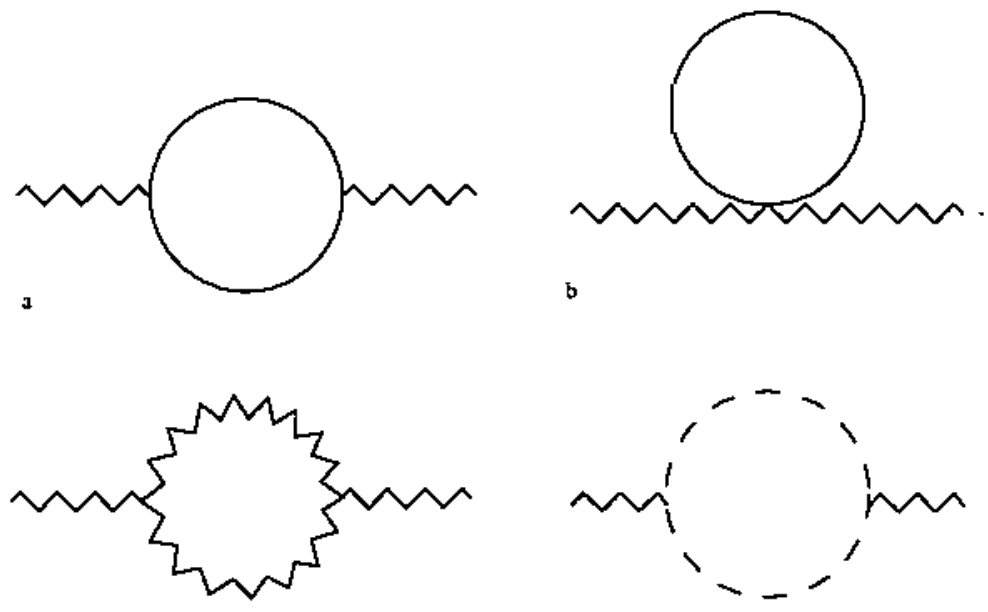

$c$

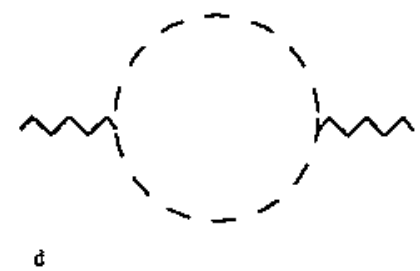

Figura 2.6: Tensor de Polarização - Teoria Relativística. 


\subsubsection{Correção de Vértice}

A correção de vértice, como a polarizaçäo do vácuo e auto-energia do campo de matéria

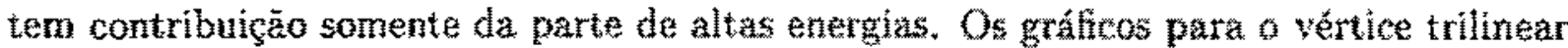

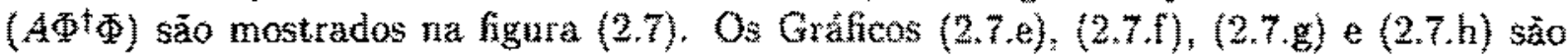
nulos devido a integração impar nos momentos ffazendo uma mudanga de variavel vemos

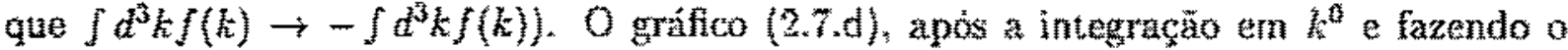
uso da parametrizaça de Feyrman, pode ser risto facilmente que tambem se anula, $\mathrm{O}$ gráfico (2.7.a) é dado analiticamente por

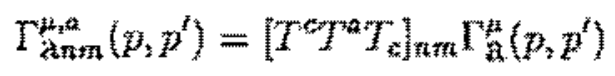

$\operatorname{com}$

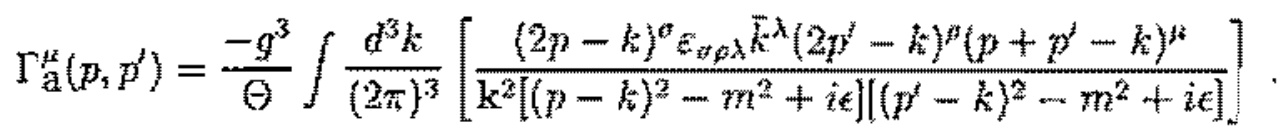

A equaçăo acima nos leva a (para $p<<m$ )

$$
\begin{aligned}
& \Gamma_{\mathrm{a}}^{0}\left(p, p^{t}\right)=\frac{i g^{3}}{4 \pi \theta m} \varepsilon_{i j} p^{i} q^{j} \\
& \Gamma_{\mathrm{a}}^{l}\left(p, p^{\prime}\right)=\frac{-i g^{3}}{8 \pi \Theta}\left[\varepsilon_{i j} \frac{p^{i} q^{j}}{m}\left(\frac{p^{i}+p^{t}}{2 m}\right)-2 z^{i l} q_{i}\left(1+\frac{p^{2}}{12 m^{2}}[2+\cos (\theta)]\right)\right]
\end{aligned}
$$

no qual $q^{i}=p^{i}-p^{i}$.

Para os gráficos $(2.7 . b)$ e $(2.7 . c)$ temos

$$
\begin{aligned}
& \Gamma_{\mathrm{bnm}}^{\mu, k}\left(p, p^{\prime}\right)=\left[\left(T^{\mathrm{a}} T^{c}+T^{c} T^{a}\right) T_{4}\right]_{n m} T_{b}^{u}\left(p, p^{\prime}\right)
\end{aligned}
$$

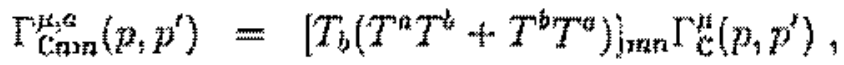

sendo

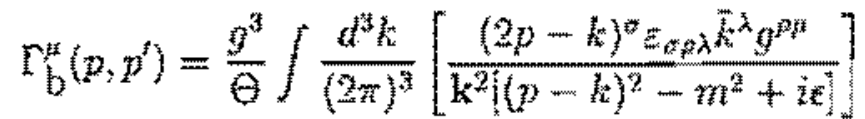

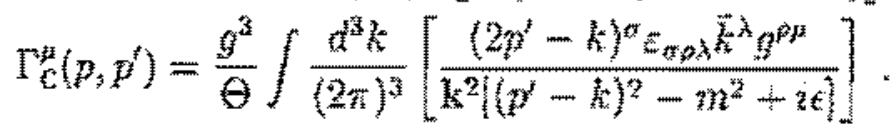

Após as integracoes obtemos até ordem $\eta^{2}$

$$
\Gamma_{\mathrm{b}}^{\mathrm{a}}\left(p, p^{\prime}\right)=\Gamma_{\mathrm{c}}^{\mathrm{p}}\left(p, p^{t}\right) \approx \mathrm{0}
$$




$$
\Gamma_{\mathbf{b}}^{l}\left(p, p^{\prime}\right)+\Gamma_{\mathrm{C}}^{l}\left(p, p^{\prime}\right)=\frac{i g^{3}}{4 \pi \Theta} \varepsilon_{i j} g^{j l} q^{i} f\left(\mathbf{p}^{2}, m^{2}\right)
$$

sendo $f\left(p^{2}, m^{2}\right)$ dado por

$$
f\left(\mathrm{p}^{2}, m^{2}\right)=\frac{\sqrt{m^{2}+\mathbf{p}^{2}}}{\sqrt{m^{2}}+\sqrt{m^{2}+\mathbf{p}^{2}}} \approx \frac{1}{2}+\frac{\mathrm{p}^{2}}{8 m^{2}} .
$$

O gráfico da figura (2.7.i) na forma analitíca é escrito como

$$
\begin{aligned}
\Gamma_{\mathrm{inm}}^{\mu, a} & =-2 i g^{3} \Theta\left[c_{f} \varepsilon^{a b c} T_{c} T_{b}\right]_{n m} \int \frac{d^{3} k}{(2 \pi)^{3}} \\
& \times\left[\Delta(k)(p+k)^{\sigma}\left(p^{\prime}+k\right)^{\rho} \varepsilon^{\mu \sigma^{\prime} \rho^{\prime}} D_{\sigma \sigma^{\prime}}(p-k) D_{\rho^{\prime} \rho}\left(p^{\prime}-k\right)\right],
\end{aligned}
$$

e será calculado explicitamente quando incorporado no gráfico de espalhamento.

As correçōes para os vértices trilineares $A A A$ e $A \eta^{*} \eta$ estāo mostradas graficamente na figura (2.8). O gráfico (2.8.d) é nulo devido a forma do propagador de gauge e o tensor $\varepsilon_{\mu \nu \alpha}$ que aparece no vértice $A A A$. O gráfico (2.8.e) é zero devido ao fato do vértice $A \eta^{*} \eta$ só conter a parte espacial do campo de gauge e que $D_{00}=D_{i j}=0$. Os gráficos (2.8.a) e (2.8.b) quando somados se cancelam[64], do mesmo modo que os tensores de polarizaçōes $\Pi_{\mathrm{C}}^{\mu \nu}$ e $\Pi_{\mathrm{d}}^{\mu \nu}$. O gráfico (2.8.c) tem divergência superficial logaritímica, no entanto, por simetria na integração ele torna-se finito. Este gráfico junto con o loop de férmions com quatro pernas externas ${ }^{6}$ de campo de gauge contribuem para completar o termo de Maxwell induzido

$$
\mathcal{L}_{\text {ind }}=\frac{1}{4}\left(\frac{g^{2}}{24 \pi m}\right) \operatorname{tr}\left(F^{\mu \nu} F_{\mu \nu}\right)
$$

\subsubsection{Espalhamento Partícula-Partícula}

Os gráficos que contribuen para a amplitude de espalhamento na ordem de árvore estão representados na figura (2.9). Usando a regras de Feynman, obtemos,

$$
\mathcal{M}_{O-\text { loop }}(\theta)=-\frac{\lambda_{\text {eff }}}{\underline{2}}-i(8 \pi) \Omega \dot{\nu}_{p} \cot (\theta / 2) \text {. }
$$

Aqui nós temos usado que

$$
\lambda_{e f f}=\lambda_{1}[I \otimes I]+\lambda_{2}\left[T^{n} \otimes T_{a}\right]
$$




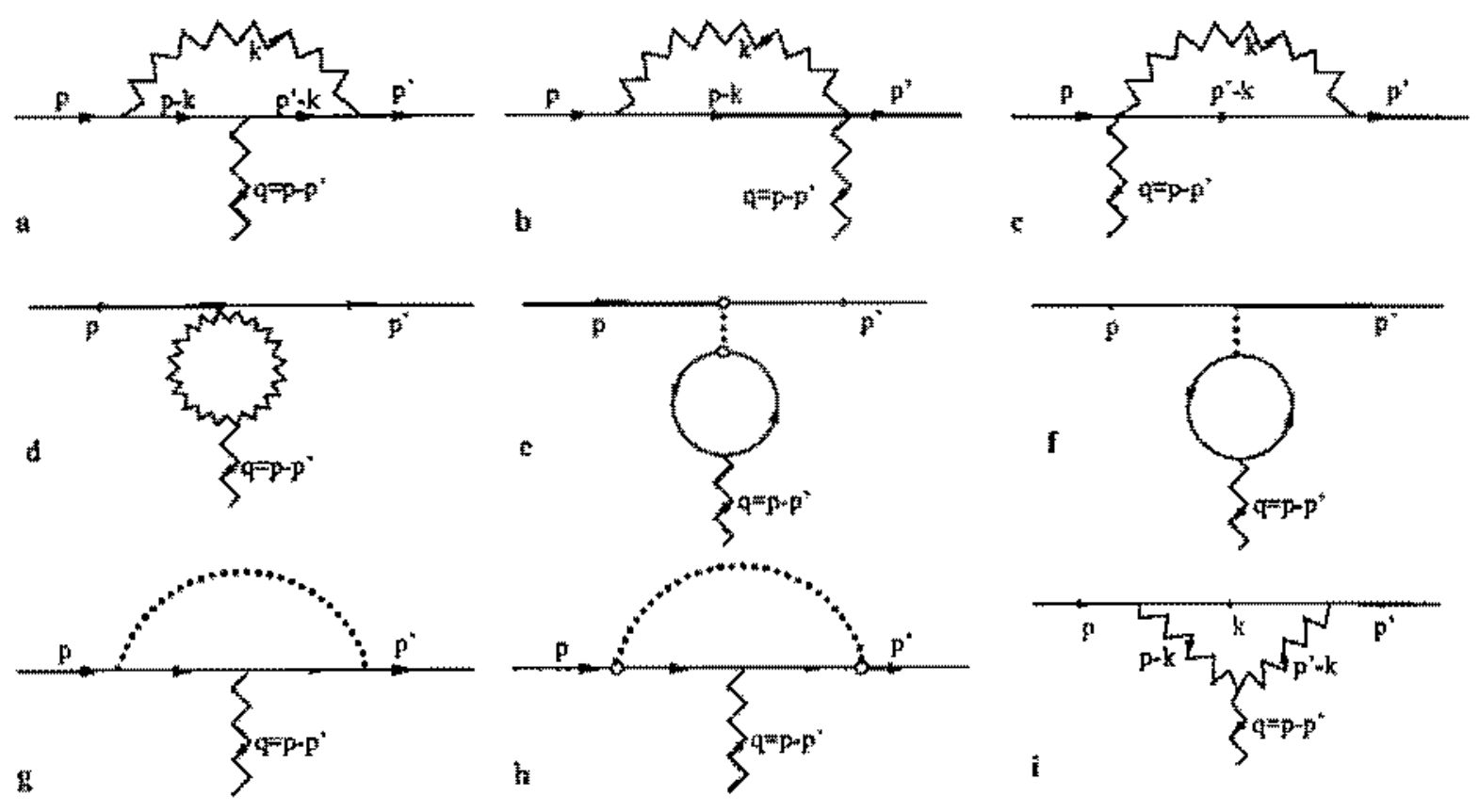

Figura 2.7: Correçào ao Vértice A Átí - Teoráa Relativística.

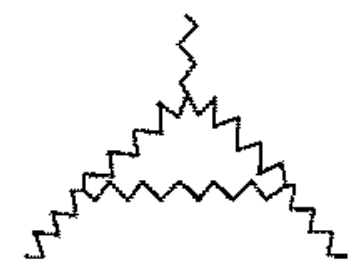

$a$

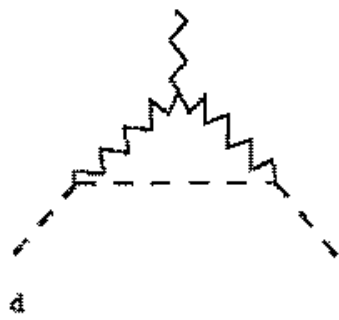

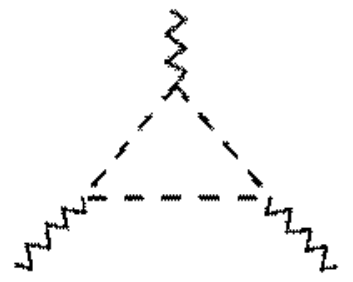

b

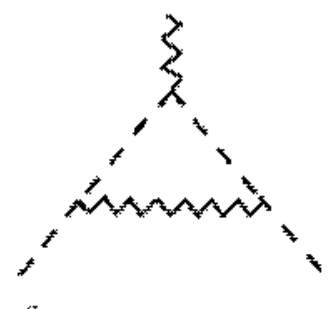

Figura 2.8: Correçào no Vertice AAA e.An* - Teoria Relativástact. 

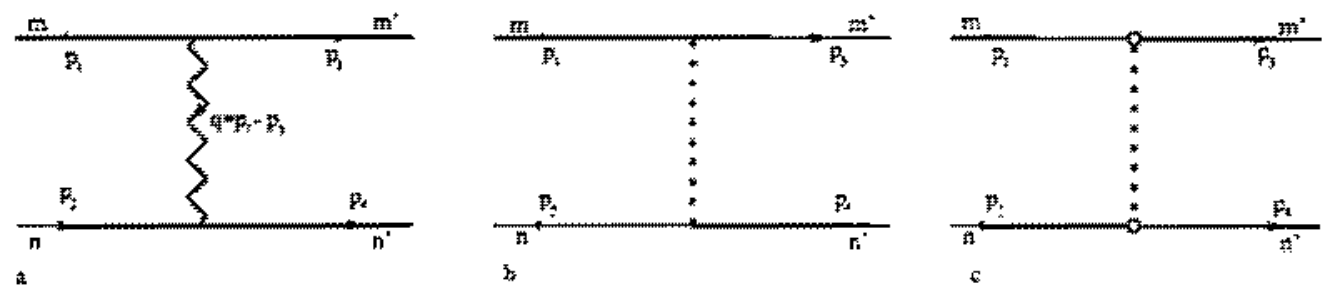

Figura 2.9: Espalhamento a Nivel de Arvore - Teoria Relativistica.

e $\Omega$ dado pela equaçăo $(2.18)$.

Vamos agora obter a contributçăo de l-loop para o espalhamento. Os gráficos que contribuirảo estão representados nas figuras (2.10),(2.11),(2.12) e (2.13). Vamos detalhar o cálculo do primeiro gräfico para deixar claro o procedimento que será usado em todos os gráficos. No apendice $\mathrm{C}$ vamos explicitar as contas do gráfico (2.11.ch) pois ele exige a introduçäo de um corte extra na variável de integração $k^{0}$. Exemplo do procedimento do corte intermediário aplicado para a teoria Abeliana pode ser visto na referência [28].

\section{Ordem $\lambda^{2}$}

Para calcular a contribuiçăo para a armplitude de espalhamento, vinda da ordem de 1-loop. vamos iniciar com contribuição de ordem $\lambda^{2}$. Os graficos desta ordem estäo mostrados na figura (2.10). Os gráficos (2.10.a1) a (2.10.a.4) dăo

$$
\mathcal{M}_{\mathrm{al}-4}(\theta)=\frac{1}{4}\left[-\lambda_{1}^{2} I \otimes I-2 \lambda_{1} \lambda_{2} T^{u} \otimes T_{a}-\lambda_{2}^{2} T^{a} T^{b} \otimes T_{b} T_{b}\right] I_{k}^{(1)}(\theta)
$$

com

$$
\begin{aligned}
f_{k}^{(1)} & =-i \int \frac{d^{k}}{(2 \pi)^{3}} \Delta(k) \Delta\left(p_{1}+p_{2}-k\right) \\
& =\frac{i}{4 \pi^{2}} \int d^{2} \mathbf{k} \int \frac{d k_{0}}{(2 \pi)} \frac{1}{\left[k_{0}^{2}-w_{k}+i \varepsilon\right]} \frac{1}{\left.\left(2 w_{p}-k_{0}\right)^{2}-w_{k}+i k\right]}=
\end{aligned}
$$

onde $w_{k}=\sqrt{\mathrm{k}^{2}+m}$. Integrando em $k_{0}$ usando o teorema dos residuos

$$
I_{k}^{(1)}=\frac{1}{16 \pi^{2}} \int_{0}^{\lambda_{0}} \frac{1}{2} d(\mathrm{k})^{2} \int_{0}^{3 \pi} d \alpha \frac{1}{u_{k}} \frac{1}{\mathrm{p}^{2}-\mathrm{k}^{2}+i \epsilon} .
$$

A integração angular é trivial, ou seja

$$
Y_{k}^{(1)}=\frac{1}{16 \pi} \int_{0}^{t_{a}} d(k)^{2} \frac{1}{w_{k} p^{3}-k^{2}+i \varepsilon} .
$$

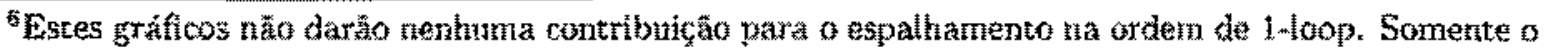
tenșor de polarizaçäo contribui nesta orien.
} 
Separando a integração restante na região de baixa e alta energia, temos

$$
\begin{aligned}
& I_{k}^{(1) L}=\frac{1}{16 \pi} \int_{0}^{\Lambda} d(\mathbf{k})^{2} \frac{1}{w_{k}} \frac{1}{\mathbf{p}^{2}-\mathbf{k}^{2}+i \epsilon} \\
& I_{k}^{(1) H}=\frac{1}{16 \pi} \int_{\Lambda}^{\Lambda_{0}} d(\mathbf{k})^{2} \frac{1}{w_{k}} \frac{1}{\mathbf{p}^{2}-\mathbf{k}^{2}+i \epsilon}
\end{aligned}
$$

$\mathrm{Na}$ parte de baixo, vamos fazer a expansão em $1 / m$, ou seja;

$$
\frac{1}{w_{k}}=\frac{1}{m}\left[1-\frac{\mathbf{k}^{2}}{2 m}+\ldots\right] \text {. }
$$

e na parte de alta energia, fazemos uma expansão em torno de $\mathrm{p}=0$, isto é

$$
\frac{1}{\mathbf{p}^{2}-\mathbf{k}^{2}}=-\frac{1}{\mathbf{k}^{2}}\left[1+\frac{\mathbf{p}^{2}}{\mathbf{k}^{2}}+\ldots\right] \text {. }
$$

Os tipos de integrais que surgem estāo mostradas no apêndice (B). Assim após as integraçōes restantes

$$
\begin{aligned}
& I_{k}^{(1) L}(\theta)=\frac{-1}{16 \pi m}\left\{\left(1-\frac{\mathrm{p}^{2}}{2 m^{2}}\right)\left[\log \left(\frac{\Lambda^{2}}{\mathrm{p}^{2}}\right)+i \pi\right]-\frac{\mathrm{p}^{2}}{\Lambda^{2}}-\frac{\mathrm{p}^{4}}{2 \Lambda^{4}}-\frac{\Lambda^{2}}{2 m^{2}}+\frac{3 \Lambda^{4}}{16 m^{4}}\right\}, \\
& I_{k}^{(1) H}(\theta)=\frac{1}{16 \pi m}\left\{\left(1-\frac{\mathrm{p}^{2}}{2 m^{2}}\right) \log \left(\frac{\Lambda^{2}}{4 m^{2}}\right)-\frac{\mathrm{p}^{2}}{2 m^{2}}-\frac{\mathrm{p}^{2}}{\Lambda^{2}}-\frac{\mathrm{p}^{4}}{2 \Lambda^{4}}-\frac{\Lambda^{2}}{2 m^{2}}+\frac{3 \Lambda^{4}}{16 m^{4}}\right\}
\end{aligned}
$$

ou seja

$$
I_{k}^{(1)}(\theta)=I_{k}^{(1) L}(\theta)+I_{k}^{(1) H}(\theta)=\frac{-1}{16 \pi m}\left\{\left(1-\frac{\mathbf{p}^{2}}{2 m^{2}}\right)\left[\log \left(\frac{4 m^{2}}{\mathbf{p}^{2}}\right)+i \pi\right]+\frac{\mathbf{p}^{2}}{2 m^{2}}\right\} .
$$

Neste ponto, podemos observar que a contribuição dominante, que vem da parte de baixa energia, equação (2.68), é a mesma que no cálculo não relativístico, equação (2.19), se o corte $A$ for identificado com $\Lambda_{N R}$. No entanto aqui, a parte de alta energia funciona como um corte natural, mantendo amplitude finita. O procedimento acima, isto é, integrando primeiro em $k^{0}$, depois na parte angular, e finalmente fazendo a separação na parte L e H, será aplicado no cálculo dos gráfico restantes. Para evitarmos a repetição nós detalharemos somente alguns pontos que achamos serem úteis no entendimento.

Os gráficos (2.10.a5) e (2.10.a6) são dados por

$$
\mathcal{M}_{\mathrm{a} 5-6}(\theta)=\frac{1}{4}\left[-n \lambda_{1}^{2} I \otimes I+\frac{\lambda_{2}^{2}}{2} T^{a} \otimes T^{\alpha}\right] I_{k}^{(2)}(\theta)
$$


onde

$$
f^{2}(0)=-i \int \frac{d^{3} k}{(2 \pi)^{3}} \Delta(k) \Delta(k-q)
$$

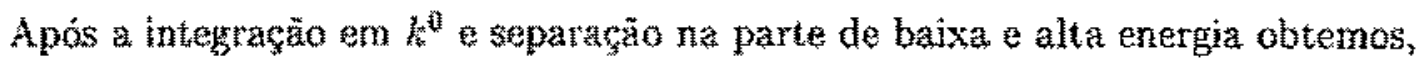

$$
\begin{gathered}
l_{k}^{(n) L_{2}}(\theta)=\frac{-1}{16 \pi m}\left[\frac{A^{2}}{m^{2}}-\frac{3 A^{4}}{4 m^{4}}\right] \\
I_{k}^{(2) H}(\theta)=\frac{-1}{16 m m}\left[2-\frac{\mathrm{p}^{2}}{3 m^{2}}(1-\cos \theta)-\frac{A^{2}}{m^{2}}+\frac{3 A^{4}}{4 m^{4}}\right] .
\end{gathered}
$$

ou seja

$$
I_{k}^{(2)}(\theta)=I_{k}^{(2) x_{h}}(\theta)+I_{k}^{(3) H}(\theta)=\frac{-1}{16 \pi m}\left[2-\frac{\mathrm{p}^{2}}{3 m^{2}}(1-\cos \theta)\right] .
$$

Os gráficos de $(2.10 . \mathrm{a})$ a $(2.10 . \mathrm{a} 10)$ levam a

$$
M_{\text {a }-\mathrm{m}}(\theta)=\frac{1}{4}\left[-\lambda_{1}^{2} I \otimes I-2 \lambda_{1} \lambda_{2} T^{a} \otimes T_{a}-\lambda_{2}^{2} T^{b} T^{a} \otimes T_{a} T_{b}\right] I_{k}^{(3)}(\theta)
$$

com

$$
f_{k}^{(3)}(\theta)=-i \int \frac{d^{3} k}{(2 \pi)^{3}} \Delta(h) \Delta\left(k+p_{2}-p_{3}\right)
$$

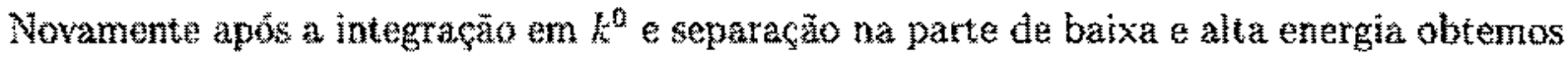

$$
\begin{aligned}
& f_{k}(3)(\theta)=\frac{-1}{16 \pi m}\left[\frac{A^{2}}{m^{2}}-\frac{3 A^{4}}{4 m^{4}}\right]
\end{aligned}
$$

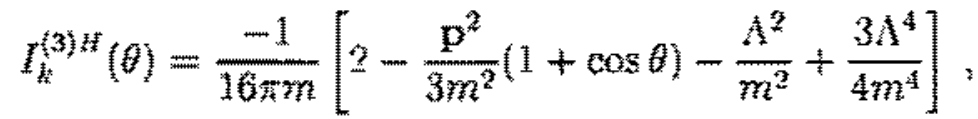

Gu seja

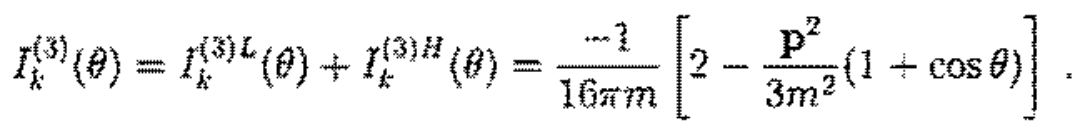

Os grafteos $(2.10 .211)$ a $(2.10 .214)$ dăo

$$
M_{a} L_{-14}(\theta)=\frac{1}{4}\left[-\lambda_{1}^{2} y \otimes I-\lambda_{1} \lambda_{2} T^{a} T_{a} \otimes I-\lambda_{1} \lambda_{2} T^{a} \otimes T_{a}-\lambda_{2}^{2} T^{a} T^{b} T_{a} \otimes T_{b}\right] I_{k}^{(2)}(\theta)
$$


Finalmente, os gráficos $(2.10 .215)$ a $(2.10 . a 18)$ fornecem $\left.M_{\mathrm{a} 15-18}(\theta)=\frac{1}{4}\left[-\lambda_{1}^{2} I \otimes I-\lambda_{1} \lambda_{2}\right] \otimes T^{a} T_{a}-\lambda_{1} \lambda_{2} T^{a} \otimes T_{6}-\lambda_{2}^{2} T_{a} \otimes T^{b} T^{a} T_{b}\right] I_{k}^{(2)}(\theta)$.

Assim temos que a soma de $(2.62),(2.71),(2.76),(2.81)$ e (2.82) nos dá

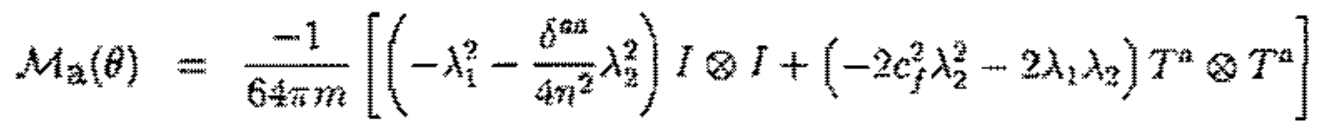

$$
\begin{aligned}
& \left\{\left(1-\frac{\mathrm{p}^{2}}{2 m^{2}}\right)\left[\log \left(\frac{4 m^{2}}{\mathrm{p}^{2}}\right)+i \pi\right]+\frac{\mathrm{p}^{2}}{2 m^{2}}\right\} \\
& -\frac{1}{64 \pi m}\left[\left(-[n+3] \lambda_{1}^{2}+\frac{\delta^{a t}}{n} \lambda_{1} \lambda_{2}-\frac{\delta^{a a}}{4 n^{2}} \lambda_{2}^{2}\right) I \otimes I\right. \\
& \left.+\left(-4 \lambda_{1} \lambda_{2}+\left[\frac{1}{2}+\frac{1}{n}-2 c_{f}^{2}\right] \lambda_{2}^{2}\right) T^{a} \otimes T^{a}\right]\left(2-\frac{p^{2}}{3 m^{2}}\right) \\
& -\frac{1}{64 \pi m}\left[\left(-[n+1] \lambda_{1}^{2}+\frac{f^{a t a}}{n} \lambda_{1} \lambda_{2}+\frac{f^{n}}{4 n^{2}} \lambda_{2}^{2}\right) 1 \otimes I\right. \\
& \left.+\left(\left[-6 c_{f}^{2}+\frac{1}{2}+\frac{1}{n}\right] \lambda_{2}^{2}\right) T^{s} \otimes T^{a}\right] \frac{\mathrm{p}^{2}}{3 m^{2}} \cos \theta \text {. }
\end{aligned}
$$

\section{Ordem $g^{4}$}

Os gráficos mais importantes com vértices de CS estảo representados na figura (2.11). Os gráficos (2.11.b1) e (2.11.b2) nos levan a

$$
\begin{aligned}
& M_{\mathrm{b} 1}(6)=-i(-g)^{\mathrm{a}}\left[T^{\mathrm{a}} T^{c} \otimes T_{2} T_{\mathrm{l}}\right] \int \frac{d^{3} k}{(2 \pi)^{3}}\left\{\Delta(k) \Delta\left(p_{1}+p_{2}-k_{4}\right)\right. \\
& {\left[\left(p_{1}+k\right)^{\mu} D_{\delta \mu}\left(k-p_{1}\right)\left(2 p_{2}+p_{1}-k\right)^{\sigma}\right]} \\
& \left.\left[\left(p_{3}+k\right)^{\mu} D_{\nu \rho}\left(k-p_{3}\right)\left(p_{2}+p_{1}+p_{4}-k\right)^{p}\right]\right\} \\
& M_{\mathrm{b} 2}(\theta)=-\left[( - g ) ^ { 4 } [ T ^ { 3 } T ^ { 2 } \otimes T _ { t } T _ { 1 } ] \int \frac { d ^ { 3 } h } { ( 2 \pi ) ^ { 3 } } \left\{\Delta\{k) \Delta\left(k+p_{2}-p_{3}\right\}\right.\right. \\
& {\left[\left(p_{1}+k\right)^{k} D_{15}\left(p_{1}-k\right)\left(k+p_{2}-p_{3}+p_{1}\right)^{d}\right]} \\
& \left.\left[\left(2 p_{2}-p_{3}+k\right)^{\nu} D_{\nu p}\left(p_{3}-k\right)\left(k+p_{3}\right)^{q}\right]\right\}
\end{aligned}
$$


Após a integração em $k^{0}$ e a separação na parte de baixa energia e alta energia, temos que

$$
\begin{aligned}
& \mathcal{M}_{\mathrm{b} 1}^{L}(\theta)=\frac{-g^{4} m}{\pi \Theta^{2}}\left[T^{c} T^{c} \otimes T_{\mathrm{a}} T_{\mathrm{c}}\right\}\left\{\left(1+\frac{\mathrm{p}^{2}}{2 m^{2}}\right)[\log [2(1-\cos \theta)]+i \pi]+2 \cos \theta \frac{\mathrm{p}^{2}}{\Lambda^{2}}\right. \\
& \left.-\cos \theta\left[\log [2(1-\cos \theta)]-\log \left(\frac{A^{2}}{\mathbf{p}^{2}}\right)\right] \frac{\mathrm{p}^{2}}{m^{2}}-\frac{\mathrm{p}^{2}}{m^{2}}-\left(1-2 \cos \theta-4 \cos ^{2} \theta\right) \frac{\mathrm{p}^{4}}{2 \mathrm{~A}^{4}}\right\} \\
& \mathcal{M}_{\mathrm{b} z}^{L}(\theta)=\frac{-g^{4} m}{4 \pi \theta^{2}}\left[T^{a} T^{c} \otimes T_{\mathrm{u}} T_{t}-2 c_{f} \varepsilon_{a b c} T^{a} T^{b} \otimes T^{c}\right]\left\{(1+\cos \theta) \frac{p^{2} \Lambda^{2}}{m^{4}}\right\} \\
& M_{b}^{\prime \prime}(\theta)=\frac{-\rho^{4} m}{\pi \Theta^{2}}\left[T^{a} T^{c} \otimes T_{a} T_{c}\right]\left\{-\cos \theta \frac{\mathrm{p}^{2}}{m^{2}} \log \left(\frac{A^{2}}{4 m^{2}}\right)-2 \cos \theta \frac{\mathrm{p}^{2}}{\Lambda^{2}}\right. \\
& \left.+\left(1-2 \cos \theta-4 \cos ^{2} \theta\right) \frac{\mathrm{p}^{4}}{2 \Lambda^{4}}-\cos \theta \frac{\mathrm{p}^{2}}{m^{2}}\right\} \\
& \mathcal{M}_{b 2}^{B}(\theta)=\frac{-g^{a} m}{4 \pi \theta^{2}}\left[T^{a} T^{c} \otimes T_{a} T_{c}-2 c y E_{a b c} T^{a} T^{b} \otimes T^{c}\right] \\
& \left\{-(1+\cos \theta) \frac{p^{2} \Lambda^{2}}{m^{4}}+2(1+\cos \theta) \frac{p^{2}}{m^{2}}\right\}
\end{aligned}
$$

usando que $\varepsilon_{\mathrm{a} b c} T^{a} T^{b} \otimes T^{*}=-2 c_{\mathrm{g}} T^{\mathrm{a}} \otimes T_{\mathrm{a}}$ temos que $M_{\mathrm{b}}=M_{\mathrm{b} 1}+M_{\mathrm{b} 2}$ fica

$$
\begin{aligned}
\mathcal{M}_{\mathrm{b}}(\theta) & =\frac{-g^{4} m}{2 \pi \Theta^{2}}\left[T ^ { \mathrm { a } } T ^ { \mathrm { c } } \otimes T _ { \mathrm { a } } T _ { \mathrm { b } } \left\{2\left(1+\frac{\mathrm{p}^{2}}{2 m^{2}}\right)[\log [2(1-\cos \theta)]+i \pi]+\right.\right. \\
& \left.-2 \cos \theta\left[\log [2(1-\cos \theta)]-\log \left(\frac{4 m^{2}}{\mathrm{p}^{2}}\right)\right] \frac{\mathrm{p}^{2}}{m^{2}}-\frac{\mathrm{p}^{2}}{m^{2}}(1+\cos \theta)\right\}+ \\
& +\frac{g^{4} m}{\pi \Theta^{2}}\left[c_{f}^{2} T^{a} \otimes T_{\mathrm{a}}\right]\left(2(1+\cos \theta) \frac{\mathrm{p}^{2}}{m^{2}}\right) .
\end{aligned}
$$

Vamos agora calcular as segunda familia de gráficos da figura (2.11). Os gráficos (2.11.c1) a (2.11.c3) individualmente apresentam divergência na integral em $k_{0}$, porém quando somados esta divergência se cancela. Analiticamente temos

$$
\begin{aligned}
\mathcal{M}_{\mathrm{c} 1}(\theta)= & \left.-g^{\mathrm{d}}\left(T^{a} T^{c}+T^{c} T^{a}\right) \otimes T_{c} T_{a}\right] \\
& \int \frac{d^{3} k}{(2 \pi)^{3}}\left\{\left[\left(p_{3}+k\right)^{\nu} \Delta(k)(p 1+k)^{k}\right] g^{\varphi \rho} D_{\alpha \mu}\left(k-p_{1}\right) D_{\nu \rho}\left(k-p_{\alpha}\right)\right\}
\end{aligned}
$$




$$
\begin{aligned}
& \operatorname{Mct}(\theta)=-g^{4}\left[T^{2} \otimes\left(T_{\mathrm{a}} T_{t}+T_{t} T_{t}\right)\right] \\
& \left.\int \frac{d^{3} k}{(2 \pi)^{3}}\left\{\left(p_{4}+k\right)^{3} \Delta(k)(p 2+k)^{3}\right] g^{* 2} D_{p k}\left(k-p_{2}\right) D_{v p}\left(k-p_{4}\right)\right\} \\
& M_{\mathrm{c} 3}(\theta)=\frac{g^{4}}{2}\left[\left(T^{\mathrm{a}} T^{\alpha}+T^{2} T^{2}\right)\left(T_{c} T_{c}+T_{k} T_{\alpha}\right)\right] \\
& \int \frac{d^{3} k}{(2 \pi)^{3}}\left\{g^{\mu \nu} g^{\sigma \rho} D_{\sigma \mu}\left(k-p_{1}\right) D_{\mu p}\left(k-p_{3}\right)\right\} \text {. }
\end{aligned}
$$

Somando estas três contribuiçōes, integrando em $k^{0}$ e na parte angular, e entāo fazendo a separação na parte de alta e baixa energia obtemos

$$
\begin{aligned}
& M_{c-m}^{2}(\theta)=\frac{-g^{4} m}{2 \pi \Theta^{2}}\left[T^{a} T^{\circ} \otimes T_{a} T_{t}\right]\left\{-2 \cos \theta \frac{\mathrm{p}^{2}}{A^{2}}+\frac{\Lambda^{4}}{8 m^{4}}+\left(1-2 \cos ^{2} \theta\right) \frac{\mathrm{p}^{4}}{\Lambda^{4}}\right. \\
& +\left(2+[1-2 \cos \theta] \frac{p^{2}}{m^{2}}\right)\left[\log \left(\frac{n^{2}}{p^{2}}\right)-[\log (2(1-\cos \theta)]]+\frac{2 p}{m^{2}}\right] \\
& +\frac{g^{4} m}{\pi \Theta^{2}}\left[c^{2} n^{n} \otimes T\right]\left\{-2 \cos \theta \frac{p^{2}}{A^{2}}+\frac{A^{4}}{8 m^{4}}+\left(1-2 \cos ^{2} \theta\right) \frac{p^{4}}{A^{4}}\right. \\
& +\left(2+[1-2 \cos \theta] \frac{\mathrm{p}^{2}}{m^{2}}\right)\left[\log \left(\frac{\mathrm{A}^{2}}{\mathrm{p}^{2}}\right)-[\log [2(1-\cos \theta)]]+\frac{2 \mathrm{p}}{m^{2}}\right\} \\
& \mathcal{M}_{\mathrm{Cl}+3}^{H}(\theta)=\frac{-g^{4} m}{2 \pi \Theta^{2}}\left[T^{\mathrm{a}} T^{c} \otimes T_{\mathrm{B}} T_{t}\right\}\left\{-2+2 \cos \theta \frac{\mathrm{p}^{2}}{\Lambda^{2}}+2 \frac{\Lambda_{0}}{m}-\frac{\Lambda^{4}}{8 m^{4}}\right. \\
& -\left(2+\left[1-2 \cos \theta \frac{\mathrm{p}^{2}}{m^{2}}\right) \log \left(\frac{\mathrm{k}^{2}}{4 m^{2}}\right)-\left(1-2 \cos ^{2} \theta\right) \frac{\mathrm{p}^{4}}{\mathrm{~A}^{4}}+\cos \theta \frac{\mathrm{p}^{2}}{m^{2}}\right) \\
& +\frac{\theta^{4} m}{m \Theta^{2}}\left[c_{j}^{2} T^{0} \otimes T_{\mathrm{w}}\right]\left\{-2+2 \cos \theta \frac{\mathrm{p}^{2}}{\Lambda^{2}}+2 \frac{\Lambda_{0}}{m}-\frac{\Lambda^{4}}{8 m^{4}}\right. \\
& -\left(2+\left[1-2 \cos \theta \frac{p^{2}}{m^{2}}\right) \log \left(\frac{A^{2}}{4 m^{2}}\right)-\left(1-2 \cos ^{2} \theta\right) \frac{p^{4}}{n^{4}}+\cos \theta \frac{p^{2}}{m^{2}}\right)
\end{aligned}
$$

isto a

$$
\begin{aligned}
M_{c 1-3}(\theta) & =\frac{-g^{4} m}{2 \pi \Theta^{2}}\left[T^{2} T^{2} \otimes T_{a} T_{t}\right]\left\{-2+(2+\cos \theta) \frac{p^{2}}{m^{2}}+2 \frac{\Lambda_{0}}{m}+\right. \\
& \left.+\left(2+[1-2 \cos \theta] \frac{p^{2}}{m^{2}}\right)\left[\log \left(\frac{4 m^{2}}{p^{2}}\right)-[\log [2(1-\cos \theta)]]\right]\right\}
\end{aligned}
$$




$$
\begin{aligned}
& +\frac{g^{4} m}{\pi \Theta^{2}}\left[c_{f}^{2} T^{2} \otimes T_{n}\right]\left\{-2+(2+\cos \theta) \frac{\mathrm{p}^{2}}{m^{2}}+2 \frac{\Lambda_{0}}{m}+\right. \\
& +\left(2+[1-2 \cos \theta] \frac{\mathrm{p}^{2}}{m^{2}}\right)\left[\log \left(\frac{4 m^{2}}{\mathrm{p}^{2}}\right)-[\log [2(1-\cos \theta)]]\right\} .
\end{aligned}
$$

Os dois últimos gráficos da figura (2.11) sāo mais problemáticos, pois estes gráficos apresentam divergência na integração em $k_{0}$ que não é cancelada pela contribuiçăo de outros gráficos, como aconteceu anteriormente. No entanto este problema pode ser contornado se introduzirmos algum tipo de regularização na integral em $h_{p}$. O resultado final, após a integraçäo na parte espacial, é finito, visto que a integral espacial restante, para o termo dependente do corte, é identicamente nula. Para deixar claro como isto acontece, nós explicitamos as contas do gráfico (2.11.c4) no apêndice C. Os gráficos $(2.11 .04)$ e (2.11.c5) säo dados por

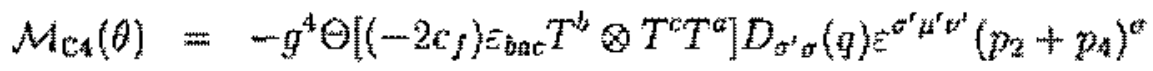

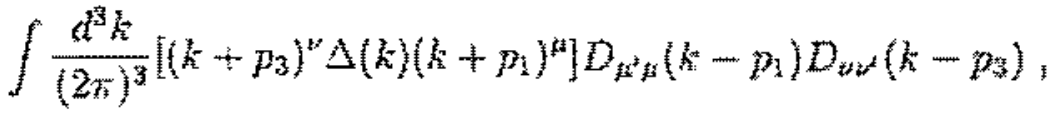

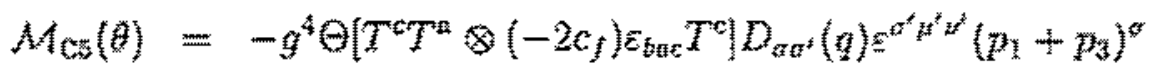

$$
\begin{aligned}
& \left.\int \frac{d^{3} k}{(2 \pi)^{3}}\left(k+p_{4}\right)^{3} \Delta(k)\left(k+p_{2}\right)^{n}\right] D_{k^{2} k}\left(k-p_{2}\right) D_{k y}\left(k-p_{4}\right) \text {. }
\end{aligned}
$$

Apớs a integração en $l^{0}$ e separação nas partes $l$ e $H$ obtemos

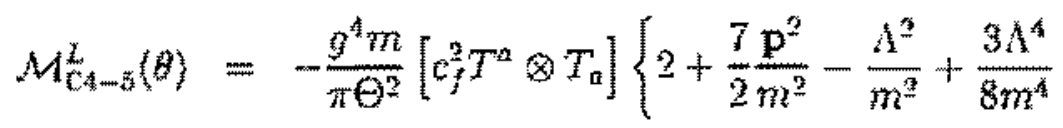

$$
\begin{aligned}
& -(4+6 \cos \theta) \frac{\mathrm{p}^{2}}{\lambda^{2}}+\left[1-4 \cos \theta-6 \cos ^{2} \theta\right] \frac{\mathrm{p}^{4}}{\AA^{4}} \\
& \left.+\left(2+[1-2 \cos \theta] \frac{\mathbf{p}^{2}}{m^{2}}\right)\left[\log \left(\frac{A^{2}}{\mathbf{p}^{2}}\right)-[\log [2(1-\cos \theta)]]\right]\right\} \text {. } \\
& \left.M_{\mathrm{C}_{4-5}}^{H}(\theta)\right)=-\frac{g^{4} m}{m \Theta^{2}}\left[\epsilon_{f}^{2} T^{2} \otimes T_{4}\right]\left\{\frac{A^{2}}{m^{2}}-\frac{3 A^{4}}{8 m^{4}}+(4+6 \cos \theta) \frac{\mathrm{p}^{2}}{A^{2}}+(2+3 \cos \theta) \frac{\mathrm{p}^{2}}{m^{2}}\right. \\
& \left.-\left[1-4 \cos \theta-6 \cos ^{2} \theta\right] \frac{\mathbf{p}^{4}}{\Lambda^{4}}-\left(2+[1-2 \cos \theta] \frac{p^{2}}{m^{2}}\right) \log \left(\frac{\Lambda^{2}}{4 m^{2}}\right)\right\}=
\end{aligned}
$$

isto $\mathrm{e}_{\mathrm{a}}$ 


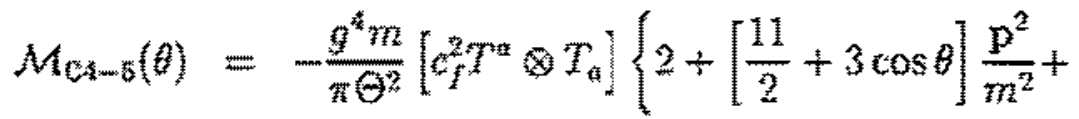

$$
\begin{aligned}
& +\left(2+[1-2 \cos \theta] \frac{p^{2}}{m^{2}}\right)\left[\log \left(\frac{4 m^{2}}{p^{2}}\right)-[\log [2(1-\cos \theta)]]\right] .
\end{aligned}
$$

Somando as contribuiçoes de (2.96) e (2.101) temos que $M_{\mathrm{c}}=\mathcal{M c l - 3}_{\mathrm{c}}+\mathcal{M}_{\mathrm{c}-\mathrm{s}} \mathrm{e}$

$$
\begin{aligned}
\mathcal{M}_{\mathrm{c}}(\theta) & =\frac{-g^{4} m}{2 m \theta^{2}}\left[T^{a} T^{c} \otimes T_{\mathrm{a}} T_{\mathrm{c}}\right]\left\{-2+(2+\cos \theta) \frac{\mathrm{p}^{2}}{m^{2}}+2 \frac{\Lambda_{0}}{m}+\right. \\
& +\left(2+[1-2 \cos \theta] \frac{\mathrm{p}^{2}}{m^{2}}\right)\left[\log \left(\frac{4 m^{2}}{\mathrm{p}^{2}}\right)-[\log [2(1-\cos \theta)]]\right\} \\
& -\frac{g^{4} m}{\pi \Theta^{2}}\left[c_{f}^{2} T^{a} \otimes T_{a}\right]\left\{4+\left[\frac{7}{2}+2 \cos \theta\right] \frac{\mathrm{p}^{2}}{m^{2}}-2 \frac{\Lambda_{0}}{m}\right\} .
\end{aligned}
$$

Podemos observar que o termo dependente de $\Lambda_{0}$ pode ser escrito como Constante[I$[\because] \Lambda_{0}$ ou seja, pode ser absorvido numa renormalização de $\lambda_{1}$.

E fácil de verifical que (a menos de constantes que poden ser absorvidas em $\lambda_{1}$ e $\lambda_{2}$ ) temos para $M_{c s}=M_{b}+M_{c}$

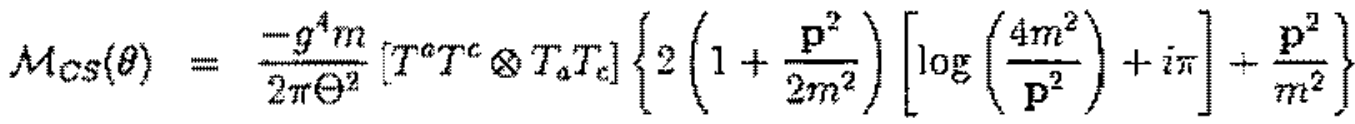

$$
\begin{aligned}
& -\frac{g^{4} m}{m \Theta^{2}}\left[e^{2} T^{n} \otimes T_{a}\right] \frac{3 \mathrm{p}^{2}}{2 m^{2}} \text {. }
\end{aligned}
$$

\section{Correçöes Radiativas}

Vamos considerar agorn amplitude que vem da insercano dos gráfos de polarizacăo do

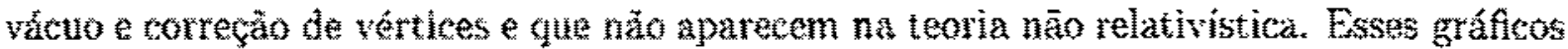
contubuiruto somente com arte de alta energia. A parte de baixa energia polinomial en $A$ e se carcela com a purte, que depende de $h$, que vem da contribuiçäo de alta

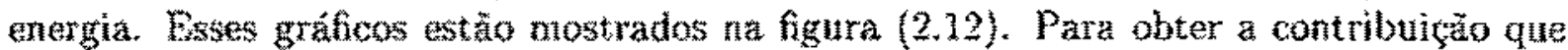
ven do tensor de polarizaçă vamos usat o resultado da equą̧ão (2.45). Assim o gráfico (2.12.P1-P2) pode ser escrito

$$
M_{p}(\theta)=\frac{g^{4} m}{4 \sigma^{2}} T^{* a} \otimes T_{n}\left[\frac{1}{3}+\frac{7}{15} \frac{\mathrm{p}^{2}}{m^{2}}+\frac{1}{5 m^{2}} \cos \theta\right\}
$$

Usando as equacoes $(2.50),(2.51), e(2.56)$ nós obtemos para os gráficos (2.12.V1) a $(2.12 .16)$ 


$$
\begin{aligned}
\mathcal{M}_{V^{\prime}}(\theta) & =-\frac{g^{4} m}{4 \pi \Theta^{2}}\left[T^{a} \otimes T_{a}\right]\left\{-4\left(\frac{1}{n}-2 c_{f}^{2}\right)+\left(\frac{22}{3} c_{f}^{2}-\frac{10}{3 n}\right) \frac{\mathbf{p}^{2}}{m^{2}}\right. \\
& \left.+\left(2 c_{f}^{2}-\frac{1}{2 n}\right) \frac{4}{3} \frac{\mathbf{p}^{2}}{m^{2}} \cos \theta\right\} .
\end{aligned}
$$

\section{Ordem $\lambda g^{2}$}

Por último, vamos calcular os gráficos da ordem $\lambda g^{2}$ mostrados na figura (2.13). Os gráficos (2.13.i) a (2.13.m) são zero devido a anti-simetria do propagador de gauge. Os gráficos (2.13.n) a (2.13.u) são zero por conjugação de carga. O cálculo dos gráficos (2.13.a) a (2.13.h), nos mostra que a parte de baixa energia é nula até ordem $\eta^{2}$. A parte de cima nos dá

$$
\mathcal{M}_{\lambda g^{2}}(\theta)=-\frac{i g^{2}}{8 \pi \Theta}\left[\frac{\delta^{a a}}{2 n}\left(\lambda_{1}+\frac{\lambda_{2}}{2 n}\right) I \otimes I+\left(\lambda_{1}+\frac{\lambda_{2}}{2 n}-4 c_{f}^{2} \lambda_{2}\right) T^{a} \otimes T_{a}\right] \frac{\mathbf{p}^{2}}{m^{2}} \sin \theta .
$$

Das equações (2.83), (2.103), (2.104), (2.105) e (2.106), temos (a menos de constantes que podem ser absorvidas numa redefinição de $\lambda_{1}$ e $\lambda_{2}$ )

$$
\begin{aligned}
\mathcal{M}_{1-\text { loop }}= & \frac{-1}{64 \pi m}\left[\left(-\lambda_{1}^{2}-\frac{\delta^{a a}}{4 n^{2}} \lambda_{2}^{2}\right) I \otimes I+\left(-2 c_{f}^{2} \lambda_{2}^{2}-2 \lambda_{1} \lambda_{2}\right) T^{a} \otimes T_{a}\right] \\
& \left\{\left(1-\frac{\mathbf{p}^{2}}{2 m^{2}}\right)\left[\log \left(\frac{4 m^{2}}{\mathbf{p}^{2}}\right)+i \pi\right]+\frac{\mathbf{p}^{2}}{2 m^{2}}\right\}+ \\
- & \frac{1}{64 \pi m}\left[\left(-[n+3] \lambda_{1}^{2}+\frac{\delta_{a}^{a}}{n} \lambda_{1} \lambda_{2}-\frac{\delta_{a}^{a}}{4 n^{2}} \lambda_{2}^{2}\right) I \otimes I+\right. \\
+ & \left.\left(-4 \lambda_{1} \lambda_{2}+\left[\frac{1}{2}+\frac{1}{n}-2 c_{f}^{2}\right] \lambda_{2}^{2}\right) T^{a} \otimes T_{a}\right]\left(-\frac{\mathbf{p}^{2}}{3 m^{2}}\right) \\
- & \frac{1}{64 \pi m}\left[\left(-[n+1] \lambda_{1}^{2}+\frac{\delta_{a}^{a}}{n} \lambda_{1} \lambda_{2}+\frac{\delta_{a}^{a}}{4 n^{2}} \lambda_{2}^{2}\right) I \otimes I+\right. \\
+ & \left.\left(\left[-6 c_{f}^{2}+\frac{1}{2}+\frac{1}{n}\right] \lambda_{2}^{2}\right) T^{a} \otimes T_{a}\right] \frac{\mathbf{p}^{2}}{3 m^{2}} \cos \theta \\
- & \frac{g^{4} m}{2 \pi \Theta^{2}}\left[T^{a} T^{c} \otimes T_{a} T_{c}\right]\left\{2\left(1+\frac{\mathbf{p}^{2}}{2 m^{2}}\right)\left[\log \left(\frac{4 m^{2}}{\mathbf{p}^{2}}\right)+i \pi\right]+\frac{\mathbf{p}^{2}}{m^{2}}\right\} \\
- & \frac{g^{4} m}{4 \pi \Theta^{2}}\left[T^{a} \otimes T_{a}\right]\left\{\left(\frac{40}{3} c_{f}^{2}-\frac{7}{15}-\frac{10}{3 n}\right) \frac{\mathbf{p}^{2}}{m^{2}}+\left(\frac{8}{3} c_{f}^{2}-\frac{1}{5}-\frac{2}{3 n}\right) \frac{\mathbf{p}^{2}}{m^{2}} \cos \theta\right\}
\end{aligned}
$$




$$
-\frac{i g^{2}}{8 \pi \theta}\left[\frac{\delta_{a}^{a}}{2 n}\left(\lambda_{1}+\frac{\lambda_{2}}{2 n}\right) I \otimes I+\left(\lambda_{1}+\frac{\lambda_{2}}{2 n}-4 c_{j}^{2} \lambda_{2}\right) T^{n} \otimes T_{a}\right] \frac{\mathbf{p}^{2}}{m^{2}} \sin \theta
$$

A seguir vamos tazer uma análise do nosso resultado, e a comparaçäo com o caso não relativistico e com o caso Abeliano.

\subsection{Análise}

Para podermos comparar com os resultados não relativísticos precisamos ajustar a normalizaçăo dos estados. No caso relativístico temos que $\left\langle\mathrm{p}^{\prime} \mid \mathrm{p}\right\rangle=2 \omega_{\mathrm{p}} \delta\left(\mathrm{p}^{\prime}-\mathrm{p}\right)$, enquanto que na normalizaçăo näo relativística a teoria näo tem o fator $2 w_{p}$. Além disso, temos um fator $f=\sqrt{\omega_{p} / m}$, onde $f^{2}$ a razäo da velocidades relativistica e não relativistica, oriundo da divisäo pelos fluxos incidertes nos cáloulos da seçāo de choque. Assim rultiplicando a amplitude (2.60) e (2.107) por

$$
f\left(\frac{1}{\sqrt{2 \omega_{p}}}\right)^{4}=\frac{1}{4 m^{2}}\left[1-\frac{3 p^{2}}{4 m^{2}}+\ldots\right]
$$

obtemos para o termo dominante em $\mathrm{p} / m$

$$
\begin{aligned}
\mathcal{M}^{D o m}(\theta) & =-\frac{1}{2}\left(\frac{\lambda_{\mathrm{e} f f}}{4 m^{2}}\right)-i \frac{2 \pi}{m} \Omega \cot (\theta / 2) \\
& +\frac{m}{16 \pi}\left[\left(\frac{\lambda_{e f f}}{4 m^{2}}\right)^{2}-\frac{16 \pi^{2}}{m^{2}} \Omega^{2}\right]\left\{\log \left[\frac{4 m^{2}}{\mathrm{p}^{2}}\right]+i \pi\right\}
\end{aligned}
$$

onde $\lambda_{e f /}$ e $\Omega$ são dados pelas equaçoes $(2.61)$ e $(2.18)$. Temos que no termo $\log \left(4 m^{2} / \mathrm{p}^{2}\right)$, o fator $4 m^{2}$ vem da parte de alta energia, e funciona como um contra-termo para a parte de baixa energía. Isto é, se considerarmos apenas a parte năo polínonial en $A$ que vem do setor de baxa energia terikmos como resultado patn o termo dominante

$$
\begin{aligned}
M^{D a t}(\theta)= & -\frac{1}{2}\left(\frac{\lambda_{t f}}{4 m^{2}}\right)-i \frac{2 \pi}{m} \Omega \cot (\theta / 2) \\
& +\frac{m}{16 \pi}\left[\left(\frac{\lambda_{\text {eff }}}{4 m^{2}}\right)^{2}-\frac{16 \pi^{2}}{m^{2}} \Omega^{2}\right]\left\{\log \left[\frac{\Lambda^{2}}{\mathrm{p}^{2}}\right]+i \pi\right\}
\end{aligned}
$$


O resultado da equaçăo acima é o mesmo obtido pela teoria não relativistica, equaçoes (2.17) e (2.23), se identificarmos $\lambda_{e f f} / \mathrm{s}^{2} \mathrm{~m}^{2}=C$ e $\Lambda \rightarrow \Lambda_{N R}$. Igualmente ao caso não relativístico, até ordem $\eta$. não teremos contribuição de 1-loop para o espalhamento $\mathrm{AB}$ se $\lambda_{e f f}^{2}=(16 \pi m)^{2} \Omega^{2}$. Lisando a forma explicita de $\lambda_{e f f}^{2}$ e $\Omega^{2}$, temos

$$
\lambda_{\text {eff }}^{2}=\left(\lambda_{1}^{2}+\frac{z_{a}^{a}}{4 n^{2}} \lambda_{2}^{2}\right)[I \otimes I]+\left(2 c_{y}^{2} \lambda_{2}^{2}+2 \lambda_{1} \lambda_{2}\right]\left[T^{a} \otimes T_{\mathrm{n}}\right]
$$

e

$$
\Omega^{2}=\frac{g^{4}}{4 \pi^{2} \Theta^{2}}\left[\frac{\delta_{a}^{s}}{4 n^{2}}[1 \otimes I]+2 c_{f}^{2}\left[T^{a} \otimes T_{\mathrm{a}}\right]\right]
$$

$O$ ponto crítico $\lambda_{e f f}^{2}=(16 \pi m)^{2} \Omega^{2}$ nos leva ao conjunto de equações

$$
\begin{aligned}
& \left(\lambda_{1}^{2}+\frac{\delta_{a}^{a}}{4 n^{2}} \lambda_{2}^{2}\right)=\frac{\delta_{a}^{a} 16 m^{2} g^{4}}{n^{2} \theta^{2}} \\
& \left(c_{i}^{3} \lambda_{2}^{2}+\lambda_{2} \lambda_{2}\right)=\frac{64 c_{f}^{2} m^{2} g^{4}}{\Theta^{2}} .
\end{aligned}
$$

As equaçòes acima apresentam quatro soluçŏes,

$$
\lambda_{1}=0 \quad \lambda_{2}= \pm \frac{8 m g^{2}}{|\theta|}
$$

ou

$$
\lambda_{1}=\frac{\delta_{a}^{a} \lambda_{2}}{4 n^{2} c_{g}^{2}} \quad \lambda_{2}= \pm \sqrt{\frac{4 n^{2} c_{f}^{4}}{4 n^{2} c^{4}+\delta_{a}^{a}}}\left(\frac{8 m g^{2}}{|\Theta|}\right)
$$

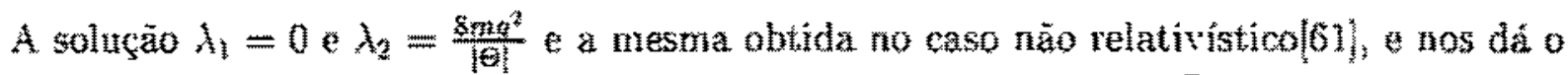
mesmo resultato da expansã̃o da solução exata até segunda ordem ${ }^{\bar{T}}$. Neste caso o termo sub-dominante (substituindo $c_{f}=-1 / 2, n=2$ e o fator de normalizaça â)

$$
\begin{aligned}
\mathcal{M}^{S u b} & =\frac{i \pi}{2 m}\left[\Omega \cot (\theta / 2)-i \frac{3}{4}|\Omega|\right] \frac{\mathrm{p}^{2}}{m^{2}} \\
& -\frac{g^{4}}{4 \pi m \Theta^{2}}\left[\frac{3}{16} I \otimes I-\frac{1}{2} T^{a} \otimes T_{a}\right] \frac{\mathrm{p}^{2}}{m^{2}}\left(\log \left[\frac{4 m^{2}}{\mathrm{p}^{2}}\right]+i \pi\right) \\
& -\frac{g^{4}}{4 \pi m \Theta^{2}}\left\{\left[\frac{1}{16} I \otimes I+\frac{2}{15} T^{n} \otimes T_{a}\right] \frac{\mathrm{p}^{2}}{m^{2}}+\left[\frac{1}{16} I \otimes I-\frac{2}{15} T^{a} \otimes T_{n}\right] \frac{\mathrm{p}^{2}}{m^{2}} \cos \theta\right\} \\
& +\frac{i g^{4}}{4 \pi m \Theta|\Theta|}\left[\frac{3}{16} I \otimes I-\frac{3}{4} T^{a} \otimes T_{a}\right] \frac{\mathrm{p}^{z}}{m^{2}} \sin \theta .
\end{aligned}
$$

\footnotetext{
${ }^{3}$ A solução com $\lambda$ : tiferente de zero nāo lesa zo resultakio da expansăo exata para o termo dominante. Na soluçāo com $\lambda_{1}=0$ e $\lambda_{a}$ negativo levaria a um Harultoniano näo limitado inferiorntente.
} 
Da equaçăo (2.107) também podemos obter facilmente o limite para a teoria Abeliana $\left(c_{f}=0, n=1, \lambda_{2}=0, \lambda_{1}=\lambda_{A}, g=\epsilon \sqrt{2}, T=i / \sqrt{2}\right)$,

$$
\begin{aligned}
\mathcal{M}_{A}(\theta)+\mathcal{M}_{A}(\theta+\pi)= & -\lambda_{A}-i \frac{8 m e^{2}}{\Theta}\left(1+\frac{\mathrm{p}^{2}}{2 m^{2}}\right) \cot \theta-\left(\frac{5 \lambda_{A}^{2}}{192 \pi m}+\frac{24 e^{2} m}{5 \pi \Theta^{2}}\right) \frac{\mathrm{p}^{2}}{m^{2}} \\
& +\left(\frac{\lambda_{A}^{2}}{32 \pi m}-\frac{2 e^{2} m}{\pi \Theta^{2}}\right)\left[\log \left(\frac{4 m^{2}}{\mathbf{p}^{2}}\right)+i \pi\right] \\
& -\frac{\mathrm{p}^{2}}{m^{2}}\left(\frac{\lambda_{A}^{2}}{64 \pi m}+\frac{e^{2} m}{\pi \Theta^{2}}\right)\left[\log \left(\frac{4 m^{2}}{\mathrm{p}^{2}}\right)+i \pi\right] .
\end{aligned}
$$

O resultado acima, multiplicado pelo fator (2.108), é essencialmente o resultado obtido na referêncial2g] para teoria abeliana.

$O$ efeito $\mathrm{AB}$ näo-Abeliano estudado neste capitulo, nos leva a uma estrutura muito mais complexa do que no caso Abeliano. Neste caso temos uma série de novos tipos de gráficos de Feynman. Estes gráficos surgem devido a presença do vértice trilinear no campo de gauge e do vertice contendo campo fantasma. As amplitudes de Feynman contendo os vértices trilineares săo importantes para, em parte cancelarem a contribuiçoes vinda dos grăficos contendo campos fantasma, e também se somarem as contribuiçôes que surgem devido a estrutura de grupo nos gráficos que igualmente estẫo presentes na teoria Abeliana.

Outra diferenga fundamental na teoria nào-Abeliana é que devido ao índice de simetria interna, o termo de troca na amplitude de spalhamento não se reduz a fazermos $\theta \rightarrow \theta+\pi$, mas é necessário também a troea nos indices do campo de matéria. Isto näo leva a um cancelarnento imediato dos termos contendo a parte angular na contribuiçäo subdominante, dada na equaçăo (2.117).

Também, no gauge de Coulomb, pelo fato do propagador do campo de gauge só depender da componente espacial, temos a possibilidade de divergências espưrias nas integraçōes em $k_{i v}$. Para o caso Abeliano essas divergencias acabam se cancelando na sorta das contribuiçöes dos grâficos que apresentam divergêncita. Xo caso não-Abeliano, além disto, é necessário introduzirmos algum tipo de regulador, para a integraçăo temporal, na amplitude que contém o vértice com três gluons. No entanto, após a integraçăo espacial as contribuiçöes dependentes do regulador se anulam, eliminando-se assim este problema da divergência $\mathrm{em} h_{0}$.

Do resultado final, obtemos que a interação quártica é fundamental para que a contribuiçào dominante reproduza o resultado da expansão da expressão exata. Os termos sub-dominantes, equaçăo (2.117), em parte sầ correçôes relativisticas ao espalhamento $\mathrm{AB}$ que nằ surgem na teoria näo relativística. Além disso, temos que a introduçăo das correçoes relativisticas induzem um fuxo magnético nào nulo fora da origem, ou sejas quando levamos en conta os efeitos relativisticos não temos mais um espalhamento $\mathrm{AB}$ puro, pois temos que levar em consideração o efeito do campo gerados pelas correçoes radiativas. Estas contribuiçoes extras correspondem, em uma Lagrangeana efetiva para 
baixos momentos, a auto-interaçōes com derivadas do campo de matéria. 

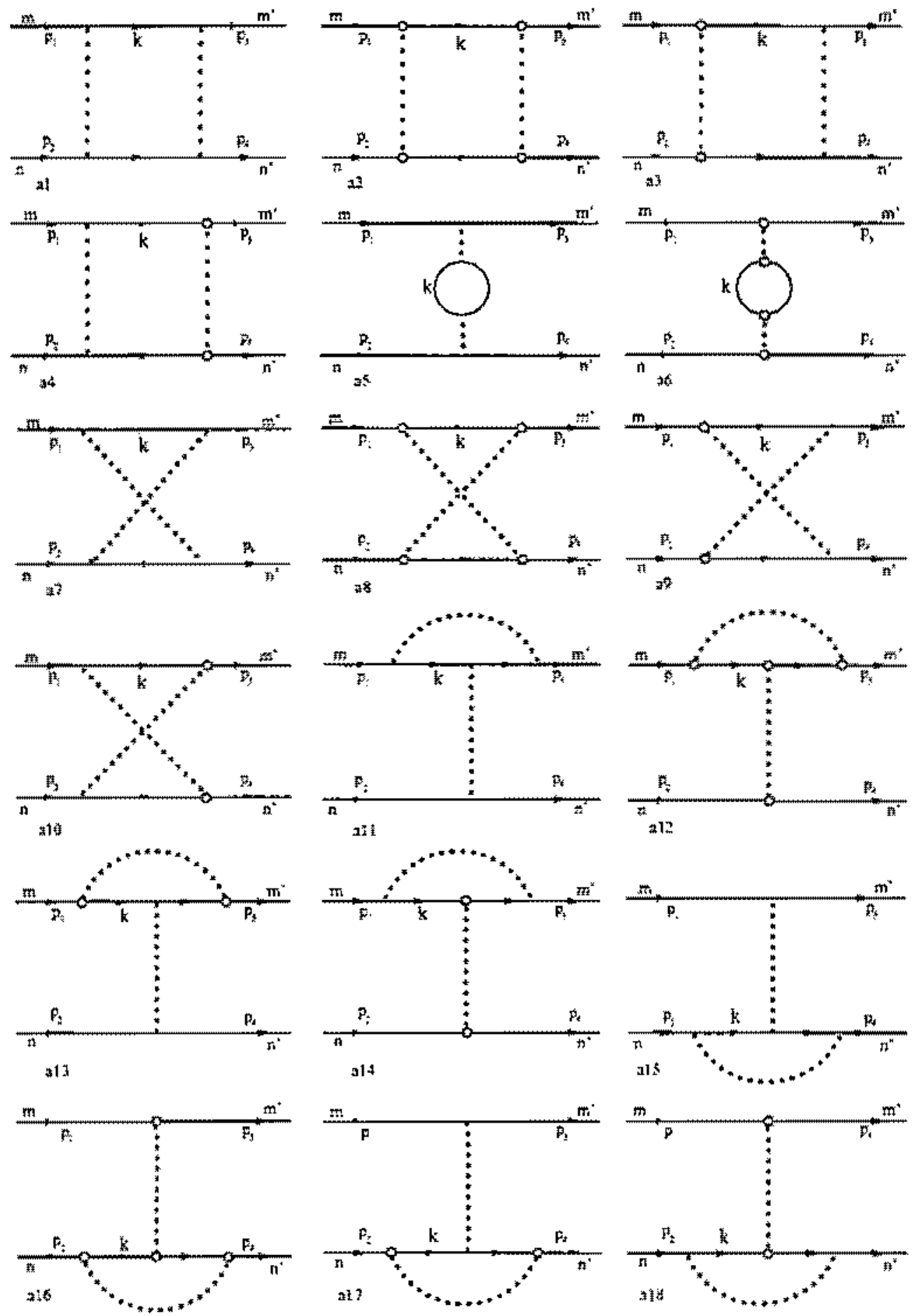

Figura 2.10; Espalhamento 1-loop - Teara Relativistica-Ordem $\lambda^{2}$. 


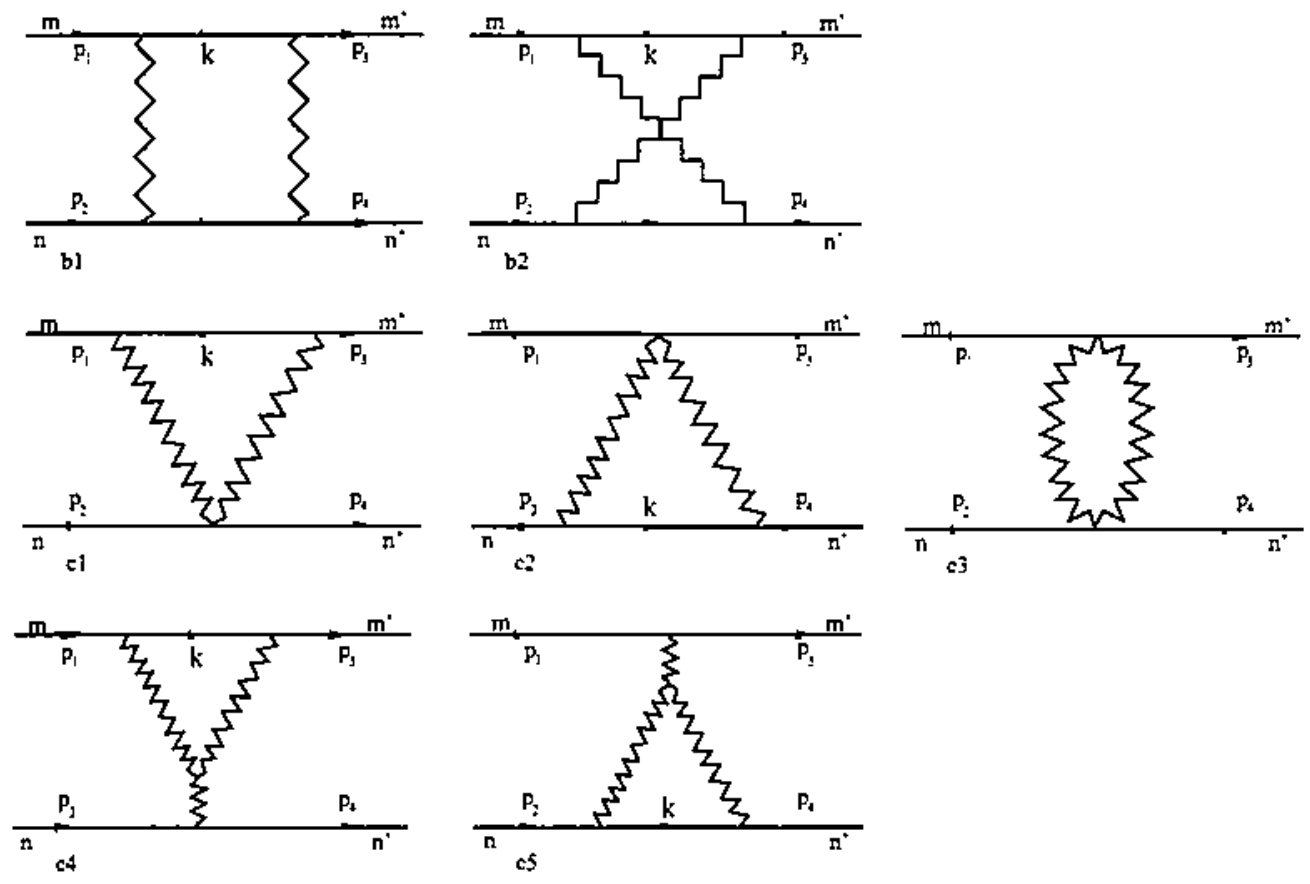

Figura 2.11: Espalhamento 1-loop-Teoria Relativistica - Ordem $g^{4}$. 

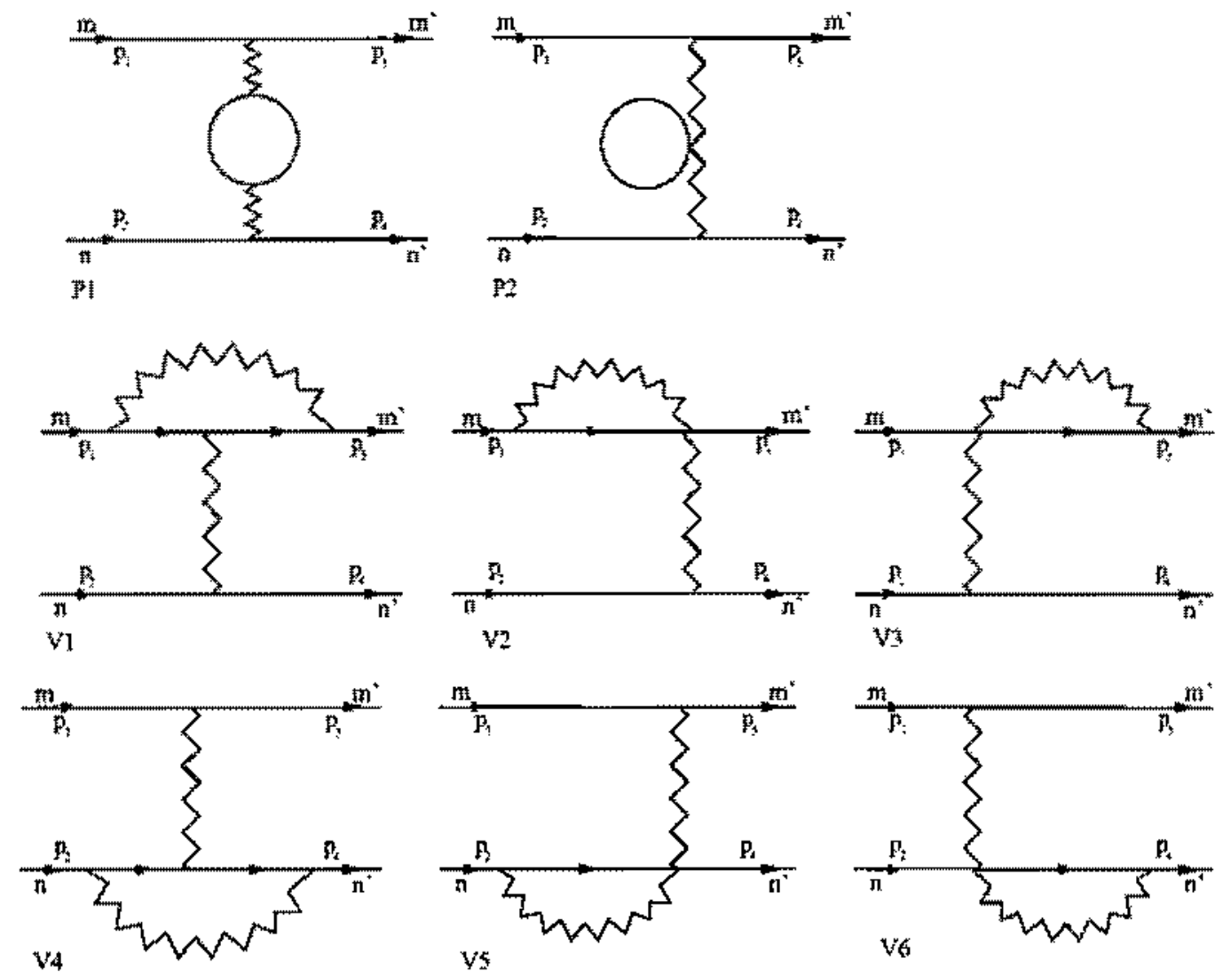

Figura 2.12: Espalhamento 1-loop - Teoria Relativistica - Polarizaçâo e Correçöes Vértice. 

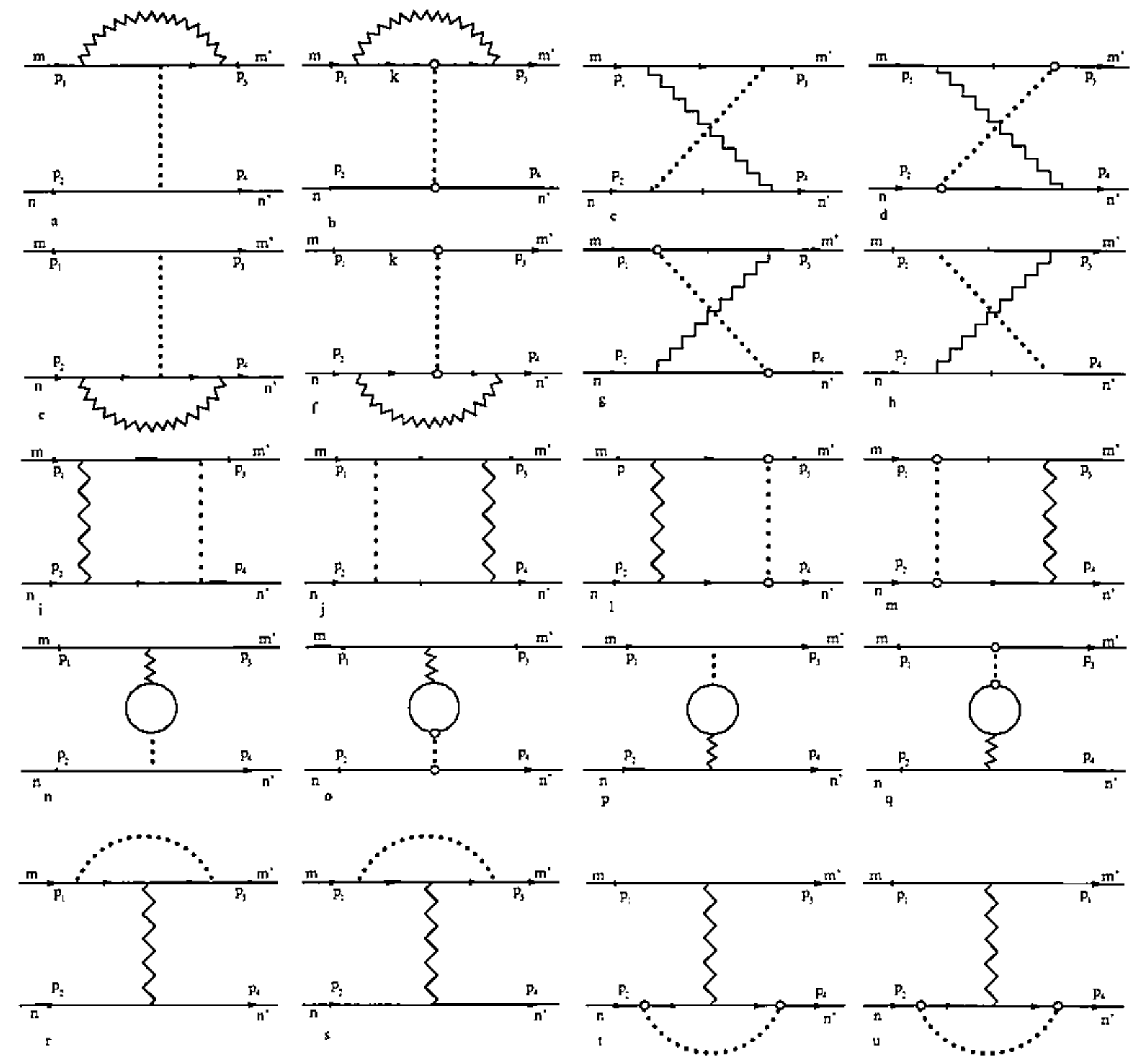

Figura 2.13: Espalhamento 1-loop - Teoria Relativistica - Ordem $\lambda g^{2}$. 


\section{Capítulo 3}

\section{Espalhamento Aharonov-Bohm Não-Abeliano para Partícula de Spin $1 / 2$}

\subsection{Introdução}

Foi mostrado no caso do efeito $A B$ Abeliano, que existem algumas características especificas devido a presença do spin $[19,23,30]$. Por exemplo, näo há necessidade de introduzirmos tum termo de auto-interação, como acontecia no caso escalar, pois o termo de Pauli faz o papel do termo de contato.

Neste capítulo vamos estender este estudo para o caso do efeito Aharonor-Bohm (AB) näo-Abeliano para a partícula de spin $1 / 2$, e ver se para este caso temos algumas características extras. Inicialmente vamos analisar o caso da teoria näo telativistica e posteriormente vamos estudar o limite para pequenos momentos na teoria relativistica. 0 proce. dimento para os cálculos é semelhante ao do capítulo anterior. Em pontos que acharmos útil nós explicitarenos alguns detalhes da conta. Yo apendice $D$ faremos explicitamente o cálculo de um dos gráficos.

\subsection{O Modelo Não Relativístico}

O sisterna de fórmions năo relativistico en $(2+1)$ dimensöes, acoplado minimamente com o campo de Chern-Simons não-tbeliano, é dado pela densidade de Lagrangeana

$$
\mathcal{L}=-\theta e^{\mathrm{a} i \lambda_{\lambda}} \operatorname{tr}\left(A_{0} \partial_{g} A_{\lambda}+\frac{2 g}{3} \cdot A_{\alpha} A_{B} A_{\lambda}\right)+\psi^{\dagger}\left[i \partial_{t}+\frac{(\vec{\nabla}-g \vec{A})^{2}}{2 m}+i g A_{0}-i \frac{g}{2 m} B\right] \psi
$$




$$
-\frac{1}{\xi} \operatorname{tr}(\vec{\nabla} \cdot \vec{A})^{2}-\eta^{* a}\left(\nabla^{2}+g f_{a b c} \mathbf{A}^{c} \cdot \nabla\right) \eta^{b},
$$

onde $\psi$ é um campo de uma componente de Lorentz que anti-comuta, transformando-se com a representaçāo fundamental do grupo de gauge $\mathrm{SU}(2)$, gerado pelas matrizes $T^{a}$, com $a=1,2,3$, e $A_{\mu}=A_{\mu}^{a} T^{a}$. Sendo $T^{a}$ matrizes anti-hermitianas $2 \times 2$, satisfazendo a álgebra de Lie

$$
\left[T_{a}, T_{b}\right]=-2 c_{f} \varepsilon_{a b c} T^{c},
$$

normalizadas por

$$
\operatorname{tr}\left(T_{a} T_{b}\right)=-\frac{1}{2} \delta_{a b} .
$$

O termo que contém a interaçāo do campo magnético $B$ é conhecido como termo de Pauli. O campo $\eta^{a}$ é o campo fantasma.

As regras de Feynman para o modelo descrito pela densidade de Lagrangeana (3.1), representadas graficamente na figura (3.1), são dadas no espaço dos momentos por

Propagador do campo de matéria

$$
S(p)_{n m}=S(p) \delta_{n m}=\frac{i}{p_{0}-\frac{\mathbf{p}^{2}}{2 m}+i \epsilon} \delta_{n m}
$$

Propagador auxiliar $\Delta_{B}^{b a}=<T B(x) A_{0}(0)>=-\frac{i}{\Theta} \delta(x) \delta^{b a}$

$\Delta_{B}^{b a}(p)=-\frac{i}{\Theta} \delta^{b a}$

Propagador para o campo fantasma

$$
G(p)_{b a}=G(p) \hat{\delta}_{b a}=\frac{-i}{\mathrm{p}^{2}} \delta_{b a}
$$

Propagador para o campo de gauge

$$
D^{\mu \nu}(k)_{b a}=D^{\mu \nu}(k) \delta_{b a}=\frac{1}{\Theta} \varepsilon^{\mu \nu \lambda} \frac{\bar{k}_{\lambda}}{\mathbf{k}^{2}} \delta_{b a}
$$

onde $\vec{k}=\left(0, k^{1}, k^{2}\right)$

\section{Vértices de Interaçāo}

$$
\begin{aligned}
& \Gamma_{n m}^{a, 0}\left(p, p^{\prime}\right)=-g T^{a} \\
& \Gamma_{n m}^{a . i}\left(p, p^{\prime}\right)=-\frac{g}{2 m}\left(T^{a}\right)_{n m}\left(p^{i}+p^{i}\right)
\end{aligned}
$$



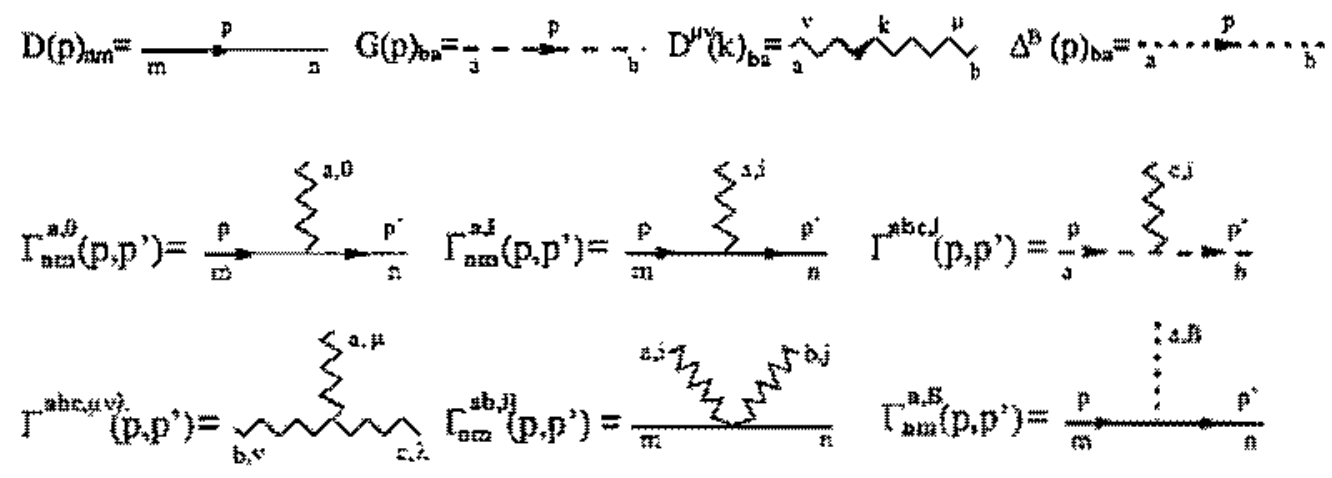

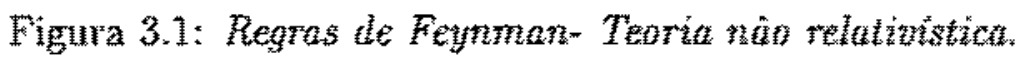

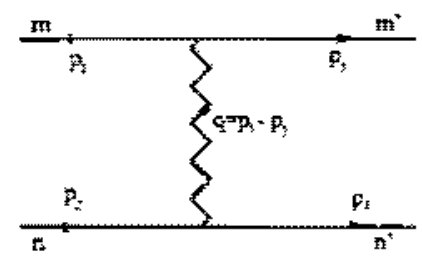

a

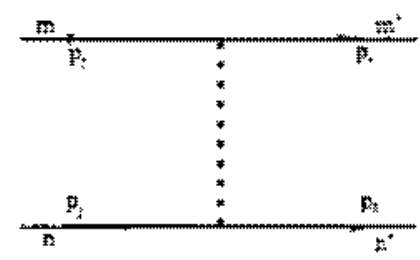

b.

Figura 3.2: Espalhamento a nível de ârvore - Teoria não relatizrstica.

$$
\begin{aligned}
& \Gamma_{n m}^{a b, i j}\left(p, p^{\prime}\right)=-i \frac{q^{2}}{2 m}\left(T^{a} T^{b}+T^{b} T^{a j}\right) g^{i j} \\
& \Gamma_{n m}^{a . B}\left(p, p^{\prime}\right)=\frac{g}{2 m}\left(T^{a}\right)_{n m}
\end{aligned}
$$

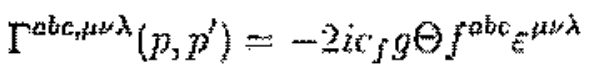

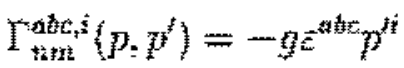

No calculo do espalhamento, vamos abstrair os indices exiemos de matéria como feito no taso escalar. isto é

$$
\left.A=n^{3} m^{7}|M(0)| m, m\right)-\left(m^{2}, n^{*}|M(\theta+m)| m, m\right)
$$

onde é o ângulo de espalhamento ${ }^{2}$. Os gráficos que contribuem para o etpalhamento a nivel de arvore, representados na figura (3.2), nos levam a

$$
M(\theta)=\frac{i g^{2}}{m \Theta}\left[T^{n} \otimes T_{n}\right]\left[1+i \frac{\sin \theta}{(1-\cos \theta)}\right]
$$

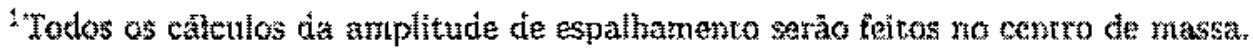


Os gráficos que contribuem (näo nulos) para o espalhamento em 1-loop estäo representados na figura (3.3). O primeiro gráfico, (3.3a), é dado por

$$
\begin{aligned}
& \mathcal{M}_{\mathrm{a}}(\theta)=\int \frac{d^{3} k}{(2 \pi)^{3}} \quad\left[\Gamma^{d, a}\left(p_{1}+p_{2}-k_{9} p_{4}\right) S\left(p_{1}+p_{2}-k\right) \Gamma^{c_{1} \nu}\left(p_{2_{1}} p_{1}+p_{2}-k\right)\right.
\end{aligned}
$$

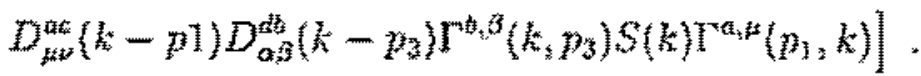

Usando as regras de Feynman a integrando em $k_{0}$ obtemos

$$
M_{\mathrm{a}}(\theta)=-\frac{4 i g^{4}}{m \Theta^{2}}\left[T^{2} T^{a} \otimes T_{\mathrm{b}} T_{a}\right] \int \frac{d^{2} \mathrm{k}}{(2 \pi)^{2}} \frac{1}{\mathrm{p}^{2}-\mathrm{k}^{2}+i \epsilon}\left[\frac{\left(\mathrm{p}_{1} \wedge \mathrm{k}\right)\left(\mathrm{p}_{3} \wedge \mathrm{k}\right)}{\left(\mathrm{k}-\mathrm{p}_{1}\right)^{2}\left(\mathrm{k}-\mathrm{p}_{3}\right)^{2}}\right]
$$

Efetuando primeiro a integração angular e depois a integraçāo no módulo de $k$, na qual introduzimos um corte ("cutoff") ultravioleta $A_{N R}$, obtemos

$$
M_{a}(\theta)=-\frac{y^{4}}{4 \pi m \Theta^{2}}\left[T^{b} T^{a} \otimes T_{b} T_{a}\right]\left\{\log \left[\frac{q^{2}}{p^{2}}\right]+i \pi\right\} .
$$

O gráfico (3.3b) analiticamente ế dado por

$$
\mathcal{M}_{\mathrm{b}}(\theta)=-\frac{g^{4}}{m^{2} \Theta^{2}}\left[T^{b} T^{2} \otimes T_{b} T_{a}\right] \int \frac{d^{3} k}{(2 \pi)^{3}} S\left(p_{1}+p_{2}-k\right) S(k) .
$$

Efetuando as integraçöes do mesmo modo que no gráfico $\mathcal{M}_{n}$ temos

$$
M_{\mathrm{b}}(\theta)=\frac{i g^{4}}{4 \pi m \theta^{2}}\left[T^{b} T^{a} \otimes T_{b} T_{a}\right]\left\{\log \left[\frac{\Lambda_{M a t}^{2}}{\mathbf{p}^{2}}\right]+i \pi\right\} .
$$

Oráfico $(3.3 \mathrm{e})$ é dado por

$$
M_{c}(\theta)=2 \int \frac{d^{3} k}{(2 \pi)^{3}}\left[\Gamma^{c d, i j} D_{0 i}^{e c}(k) D_{j 0}^{d \xi}(k+q) \Gamma^{2,0} \Gamma^{0,0}\right],
$$

no quăl o fator 2 é devido ao gráfico invertido, obtido pela troca da linha fermóonica inferior com a superior. Após a integração em $k_{0}$ temos

$$
\mathcal{M}_{c}(\theta)=\frac{i g^{4}}{2 m \Theta^{2}}\left[\left(T^{a} T^{b}+T^{b} T^{a}\right) \otimes T_{b} T_{a}\right] \int \frac{d^{2} \mathbf{k}}{(2 \pi)^{2}} \frac{\mathbf{k}(\mathbf{k}+\mathrm{q})}{\mathrm{k}^{2}(\mathrm{k}+\mathrm{q})^{2}} .
$$

Efetuando as integracoes espaciais e usando as relagöos entre as matrizes $T$,

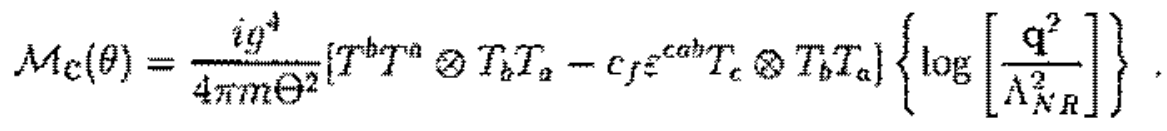


Finalmente o gráfico $(3,3 d)$, que contểm o vêttice com trểs campos de gauge, corresponde a

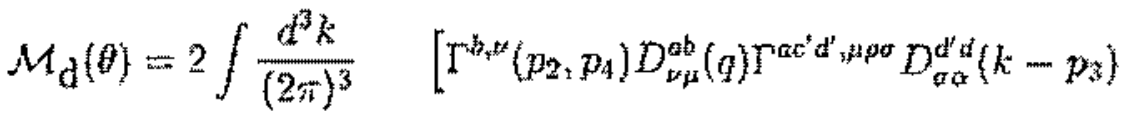

$$
\begin{aligned}
& \left.\Gamma^{d, a}\left(k, p_{3}\right) S(k) \Gamma^{c, 3}\left(p_{1}, k\right) D_{B \rho}^{c c^{\prime}}\left(k-p_{1}\right)\right] \text {. }
\end{aligned}
$$

Integrando em $h_{0}$ temos

$$
M_{\mathrm{d}}(\theta)=-\frac{2 i g^{4}}{m \Theta^{2}}\left[c_{j} e^{\mathrm{cot} t} T_{\mathrm{c}} \otimes T_{b} T_{\mathrm{a}}\right] \int \frac{d^{2} \mathrm{k}}{(2 \pi)^{2}} \frac{\left[\mathrm{q} \wedge \mathrm{k}-\mathrm{p}_{1} \wedge \mathrm{p}_{3}\right](\mathrm{q} \wedge \mathrm{k}\}}{\mathrm{q}^{2}\left(\mathrm{k}-\mathrm{p}_{1}\right)^{2}\left(\mathrm{k}-\mathrm{p}_{3}\right)^{2}}
$$

que nos leva a

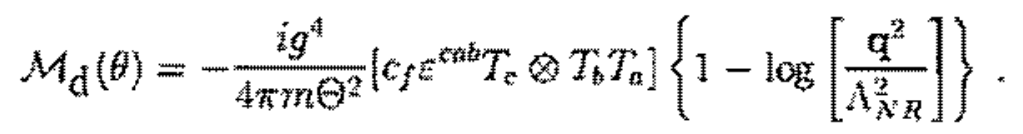

Assim, obtemos das equaçöes (3.19), (3.21), (3.24) e (3.27)

$$
M_{1-l o o p}(\theta)=-\frac{i g^{4}}{4 \pi m \Theta^{2}}\left[c_{f} c^{c a b} T_{r} \otimes T_{i} T_{b}\right]=-\frac{i c_{f}^{2} g^{4}}{2 \pi m \Theta^{2}}\left[T^{n} \otimes T_{n}\right] .
$$

O termo dado na equaçäo acima é devido a regularização empregada. Realmente, lembrando que a divergência dos gráficos é logaritimica, então nós podemos ter uma certa ambiguidade na constante finita. Por outro lado, se tivessemos usado a regularizaçăo dimensional como feito na referência [22] para o caso escalar, não teriamos o termo (3.28). Este fator constante pode ser eliminado nor uma redefiniçăo do torte $A_{A} R$ na equaçăo (3.27) ou pela adiça de urn contra- termo da forma $L_{C T}=c_{1} B \psi^{\dagger} b+c_{2}\left(\psi^{4} w^{\dagger}\right)^{2}$ na densidade de Lagrangeana inicial com $c_{1}$ e $c_{2}$ ajustados de modo que fator do monento magnético seja $\mathrm{g} / 2 \mathrm{~m}$ e o resultado final do espalhamento continue finito até 1-loop. Fste problema aparece no caso da teoria năo relativistica porque os gráficos sào dependentes do corte introduzido. No caso da teoria relativistica, a menos da função de dois pontos, os gráficos individualmente são finitos e independentes do corte introduzido para regularizar a teoria, assim nāo teremos a ambiguidade no termo finito. Podemos tambén observar no cálculo de $M_{\mathrm{b}}$ : equação (3.21) e na amplitude (2.19), que a interação $\hat{\psi}^{\dagger} B \psi \mathrm{faz}$ o mesmo papel da interaçào $\left(\phi^{\dagger} \phi\right)^{2}$ no caso estalar. 

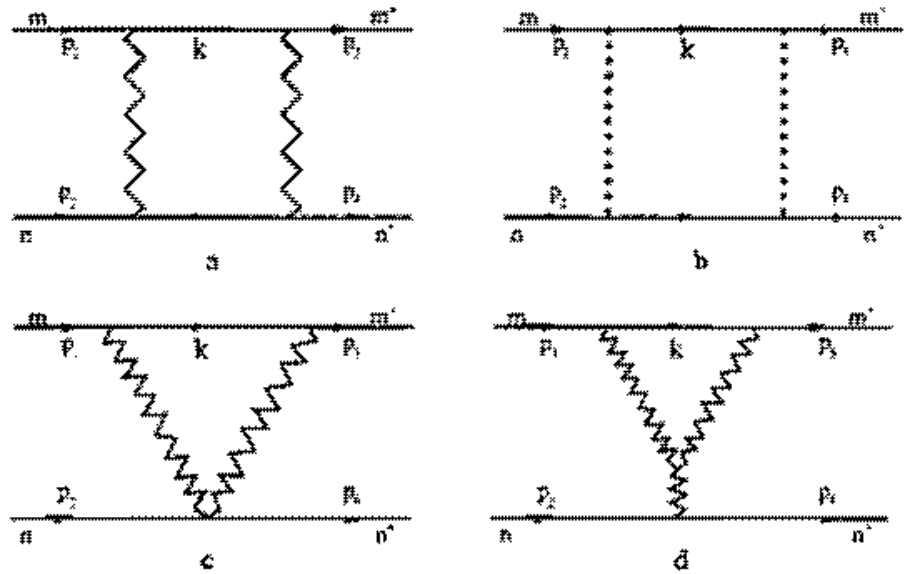

Figura 3.3: Espalhamento 1-loop - Teoría näa relafizunstica.

\section{$3.3 \quad \mathrm{O}$ Modelo Relativístico}

Como no caso escalar vamos tratar o problema através do cálculo relativistico e fazendo a separaçăo na regiôes de alta e baixa energia. A Lagrangeana do modelo è dada por

$$
\begin{aligned}
& \mathcal{L}=-\Theta \sigma^{\alpha 3 \lambda_{t r}}\left(A_{\alpha} \partial_{\beta} \cdot A_{\lambda}+\frac{2 g}{3} A_{\alpha} \cdot A_{B}, A_{\lambda}\right)+i \Psi(D D-m) \Psi \\
& -\frac{1}{\xi} \operatorname{tr}(\ddot{\nabla} \cdot \vec{A})^{2}-\eta^{\circ}\left(\nabla^{2}+g \int_{a b c} A^{c} \cdot \nabla\right) \eta^{b} \text {. }
\end{aligned}
$$

onde $\Psi$ é um campo de Dirac transformando-se com a representaçăo fundamental do grupo de gauge $S U(2)$, gerado pelas matrizes $T^{a}$, com $a=1,2,3, A_{\mu}=A_{\mu}^{a} T^{a}$ \& $D_{p}=\partial_{k j}+g A_{\mu}$. As matrizes de Dirac săo dadas por

$$
r^{0}=\sigma^{3} \quad \gamma^{i}=i \sigma^{2} \quad \gamma^{2}=i \sigma^{2}
$$

sendo $\sigma$ as matrizes de Pauli

$$
\sigma^{1}=\left(\begin{array}{ll}
0 & 1 \\
1 & 0
\end{array}\right) \quad \sigma^{2}=\left(\begin{array}{cc}
0 & -i \\
i & 0
\end{array}\right) \quad \sigma^{3}=\left(\begin{array}{cc}
1 & 0 \\
0 & -1
\end{array}\right) .
$$

O que nos leva as relaçoes $\operatorname{tr}\left(\gamma^{n}\right)=0$ e $\gamma^{\alpha} \gamma^{\beta}=g^{\alpha \hat{s}}-i g^{\alpha \beta^{\alpha}} \gamma_{p}$.

As soluções de onda plana para equaçăo de Dirac live

$$
\begin{array}{r}
\Psi^{t}(x)=u(p) e^{-i p x} \\
\Psi(x)=v(p) e^{i p x}
\end{array}
$$


nos dão[67] para a solução de energia positiva e negativa

$$
\begin{aligned}
& u(\mathbf{p})=\left(\frac{w_{p}+m}{2 w_{p}}\right)^{1 / 2}\left(\begin{array}{c}
1 \\
\frac{-i p_{+}}{w_{p}+m}
\end{array}\right), \\
& v(\mathbf{p})=\left(\frac{w_{p}+m}{2 w_{p}}\right)^{1 / 2}\left(\begin{array}{c}
\frac{i p_{-}}{w_{p}+m} \\
1
\end{array}\right),
\end{aligned}
$$

$\operatorname{com} w_{p}=\sqrt{\mathbf{p}^{2}+m}, p_{ \pm}=p^{(1)} \pm i p^{(2)}$, normalizadas por

$$
\begin{aligned}
u^{\dagger}(\mathbf{p}) u(\mathbf{p}) & =v^{\dagger}(\mathbf{p}) v(\mathbf{p})=1 \\
\bar{u}(\mathbf{p}) u(\mathbf{p}) & =\bar{v}(\mathbf{p}) v(\mathbf{p})=\frac{m}{u_{p}}
\end{aligned}
$$

Para pequenos momentos $(\mathrm{p}<<m)$ temos que

$$
\begin{aligned}
& \bar{u}\left(\mathbf{p}_{\mathbf{3}}\right) \gamma^{0} u\left(\mathbf{p}_{\mathbf{1}}\right)=1+\mathcal{O}\left(\frac{\mathbf{p}^{2}}{m^{2}}\right) \\
& \bar{u}\left(\mathbf{p}_{\mathbf{3}}\right) \gamma^{i} u\left(\mathbf{p}_{\mathbf{1}}\right)=\frac{1}{2 m}\left[P^{i}+i \bar{\varepsilon}^{i j} q^{j}\right]+\mathcal{O}\left(\frac{\mathbf{p}^{2}}{m^{2}}\right),
\end{aligned}
$$

no qual $P^{i}=p_{1}^{i}+p_{3}^{i}, q^{i}=p_{1}^{i}-p_{3}^{i}$ e $\left|p_{1}\right|=\left|p_{3}\right|=|\mathbf{P}|$.

As regras de Feynman, representadas na figura (3.4), sāo dadas por

Propagador do campo de matéria

$S(p)_{n n}=S(p) \delta_{n m}=\frac{i(\not p+m)}{p^{2}-m^{2}+i \epsilon} \delta_{n m}$

Propagador para o campo fantasma

$$
G(p)_{b a}=G(p) \delta_{b a}=\frac{-i}{\mathrm{p}^{2}} \delta_{b a}
$$

Propagador para o campo de gauge

$$
D^{\mu \nu}(k)_{b a}=D^{\mu \nu}(k) \delta_{b a}=\frac{1}{\Theta} \varepsilon^{\mu \nu \lambda} \frac{\bar{k}_{\lambda}}{\mathbf{k}^{2}} \delta_{b a}
$$

onde $\bar{k}=\left(0, k^{1}, k^{2}\right)$ 


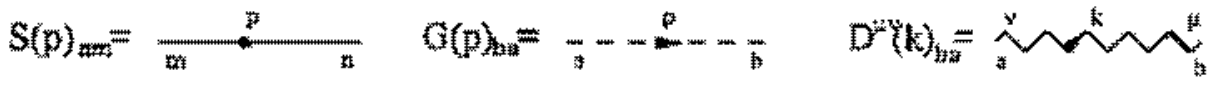

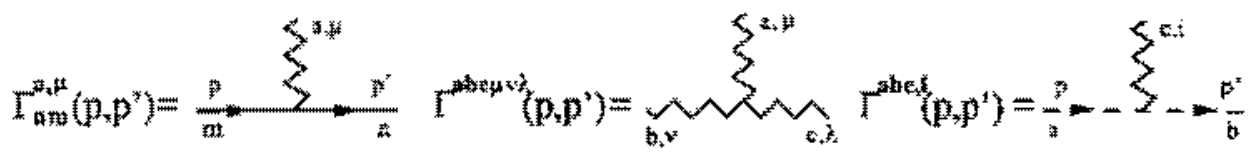

Figura 3.4: Regras de Feynmon- Teoria Relatainstita.

\section{Vértices de Interaçäo}

$$
\begin{aligned}
& \Gamma_{m y a}^{a_{z} k}\left(p, p^{k}\right)=-g\left(T^{a}\right)_{n m i}\left(\gamma^{j}\right)
\end{aligned}
$$

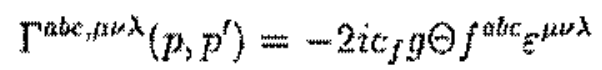

$$
\begin{aligned}
& \Gamma_{n z h t}^{a b c, i}\left(p, p^{\prime}\right)=-g e^{a b c} p^{i}
\end{aligned}
$$

Nas próximas seções iremos calcular a correçăo de 1-loop e analisar a estrutura ultravioleta da teoria. Os gráficos que näo contén o campo de matéria não dão nenhuma contribuiçäo[64], como no caso escálar. Assinu nos restringirenos a análise dos gráficos restantes.

\subsubsection{Correção para a Auto-Energia}

A correçäo de 1-loop para a funçäo de dois pontos do campo de matéria, representada na figura (3.5), edada por

$$
\begin{aligned}
& \Sigma(p)=-\int \frac{t^{3} k}{(2 \pi)^{3}}\left[\Gamma^{a, k}(p+k, p) S(p+k) \Gamma^{b, t}(p, p+k) D_{v_{j}}^{t b}(k)\right] \\
& =\frac{i g^{2}}{\theta}\left[T^{n} T_{t}\right] \int \frac{d^{3} k}{(2 \pi)^{3}} \frac{\left[m^{3}(h+k+m)\right.}{\left[(y+k)^{2}-m^{2}+i \hbar \bar{k}^{2}\right.}
\end{aligned}
$$

no qual o sinal - introduzido de modo que $\mathcal{S}^{-1}(p)=p-m \div+1 \mathrm{~s}$. Usando as propriedades da matrizes $\gamma$ podenos escrever o numerador do integrando como

$$
N=-2(\mathrm{p}+\mathrm{k}) \mathrm{Ak}+\mathrm{m} m \mathrm{k} \text {. }
$$




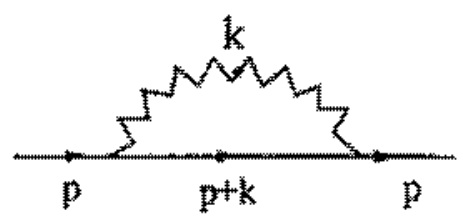

Figura 3.5: Abto-Energía do Férmion.

Integrando en $k_{0}$ e na parte angular obtemos

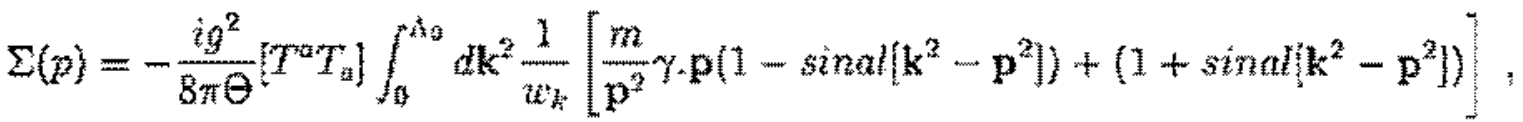

com $w_{k}=\sqrt{k^{3}+m}$. Separando nu parta de baixa e alta energia

$$
\begin{aligned}
& \Sigma^{L}(p)=-\frac{i g^{2}}{2 \pi \Theta}\left[T^{n} T_{n}\right]\left\{\gamma \mathrm{p} \frac{m}{\mathrm{p}^{2}}\left(u_{p}-|m|\right)+\sqrt{\Lambda^{2}+\mathrm{p}^{2}}-w_{p} \mid\right\} \\
& \Sigma^{H}\langle p\rangle=-\frac{i g^{2}}{2 \pi \Theta^{2}}\left[T^{a} T_{a}\right]\left\{\sqrt{\Lambda_{0}^{2}+m^{2}}-\sqrt{\Lambda^{2}+m^{2}}\right\}
\end{aligned}
$$

ou seja, para $\Lambda_{0} \rightarrow \infty$ e con $m$ positivo,

$$
\Sigma(p)=\Sigma^{L}(p)+\Sigma^{n}(p)=-\frac{i g^{2}}{2 \pi \Theta}\left[T^{\alpha} T_{a}\right\}\left\{\frac{m \gamma \cdot \mathrm{p}-\mathrm{p}^{2}}{w_{p}+m}-m+\Lambda_{0}\right\} .
$$

O propagador completo é dado por

$$
S(p)=\frac{i}{\not p-m+i \Sigma_{R}}
$$

com $\Sigma_{R}=\Sigma+i \delta m-i \delta Z \not h$, sendo $\delta m$ e $\delta Z$ contra-termos de massa e funçâo de onda. Como a forma de $\Sigma$ não é covariante de Lorentz, entäo, para determinarmos o propagador: vamos, do mesmo modo aplicado para n eletrodinämica em $(3+1) \mathrm{D}$ no gauge de Conlomb[68], assumir que o seu inverso tenha a forma

$$
S^{(-1)}(p)=-m+i \Sigma_{R}=a m+b \gamma p+c \xi \gamma m
$$

com $n=(1, \overrightarrow{0}), \xi=p n=w_{p}$, e $a, b$ e $c$ funçoes de $p^{3}$ que são calculadas ordem a ordem pelos gráficos de Feynman. Usando que $S^{-1} S=i$ podemos inverter (3.54)

$$
S(p)=i \frac{a m-b(p)-c \xi n}{m^{2} \Delta\left(p^{2} \xi \xi\right)}
$$

com

$$
\Delta\left(p^{2}, \xi\right)=a^{2}\left(p^{2}, \xi\right)-b^{2}\left(p^{2}, \xi\right)\left[\frac{p^{2}}{m^{2}}\right]-c^{2}\left(p^{2}, \xi\right)\left[\frac{\xi^{2}}{m^{2}}\right] \ldots 2 b\left(p^{2}, \xi\right) c\left(p^{2}, \xi\right)\left[\frac{\xi^{2}}{m^{2}}\right]
$$


e a condição que $\Delta\left(p^{2}=m^{2}, \xi\right)=0$, ou seja $m$ é a massa física.

Usando a forma explícita de $\Sigma(p)$ nós obtemos que

$$
\begin{aligned}
& a\left(p^{2}, \xi\right)=\left[-1-\frac{\delta m}{m}+\frac{\alpha}{m}\left(\xi-\Lambda_{0}\right)\right] \\
& b\left(p^{2}, \xi\right)=\left[1-\frac{\alpha}{m}(m-\xi)+\delta Z\right] \\
& c\left(p^{2} \xi\right)=\left[\frac{\alpha}{m} \frac{m^{2}}{p^{2}}(m-\xi)\right]
\end{aligned}
$$

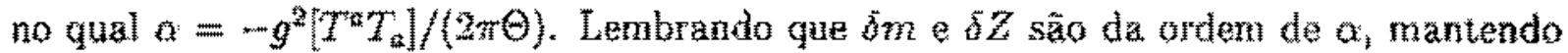
termos ate primeira ordem e usando a condiça $\Delta\left(\eta^{2}=m^{2}, \xi\right)=0$, obtanos que $\delta m / m=$ $\alpha\left(1-h_{0} / m\right)+2 Z$. Assim temos que

$$
\Delta\left(p^{2} \xi \xi\right)=\frac{p^{2}-m^{2}}{m^{2}}(-1-2 \delta Z)
$$

Logo o propagador completo

$$
\begin{aligned}
S(p) & =\frac{-a m+b \gamma \cdot p+c \xi \gamma \cdot n}{\left[p^{2}-m^{2}\right](1+2 \delta Z)} \\
& =i \frac{(p+m)+\alpha\left(m-w_{p}\right)\left[1+\frac{m}{p^{2}} \gamma \cdot p\right]}{\left(p^{2}-m^{2}\right)(1+2 \delta Z)}
\end{aligned}
$$

Temos que o propagador renormalizado é definido $S(p)=Z_{2} S_{R}(p)$. Como podemos ver na equaçäo (3.61), o segundo terno do propagador $S(p)$ tem uma dependéncia cornplicada

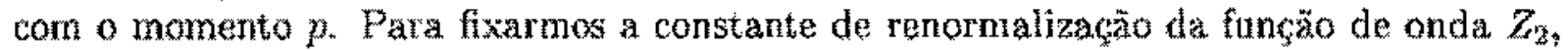
vamos usar mesma condiçüo sugerida em [68], ou seja que o propagador renomalizado no referencial de repouso seja igual ao propagador livre, isto $S_{R}\left(p_{0}, \mathrm{p}=0\right)=S\left(p_{0}, \mathrm{p}=0\right)$. Assim obtemos $Z z=1$ ou $\delta Z=0$, entäo podemos escrever

$$
S_{n}(p)=i \frac{(f+m)+\alpha\left(n-u_{p}\right)\left[p+\frac{m^{2}}{p^{2}} \gamma p\right)}{\left(p^{2}-m^{2}\right)}
$$

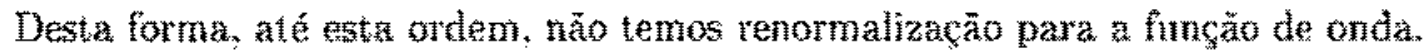

\subsubsection{Tensor de Polarizaçāo}

O único grafico que contribui para o tensor de polarizacão esta rapresentado da figura (3.6). Observemos que este graffeo é independente de gatuge[1] a apresenta divergêneia 


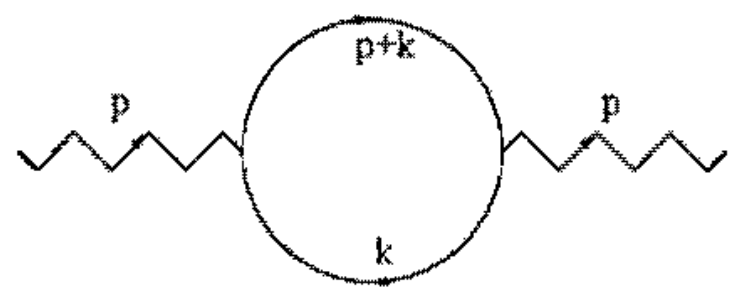

Figura 3.6: Tensor de Polarizaçäo

linear no ultravioleta. No entanto, esta divergencia pode ser eliminada se usarmos alguma regularização invariante de gauge. Analiticamente o gráfico é dado por

$$
\begin{aligned}
\Pi^{\mu k x} & =(-1) \int \frac{d^{3} k}{(2 \pi)^{3}} \operatorname{tr}\left[\Gamma^{a, 3}(k, k+q) S(k+q) \Gamma^{b, v}(k+a, k) S(k)\right] \\
& =g^{2} \operatorname{tr}\left[T^{a} T^{b}\right] \int \frac{d^{3} k}{(2 \pi)^{3}} \frac{\operatorname{tr}\left[\gamma^{\mu}(k+d+m) \gamma^{4}(k+m)\right]}{\left.\left[(k+q)^{2}-m^{2}\right] \mid k^{2}-m^{2}\right]} .
\end{aligned}
$$

0 integrando e o mesmo do taso abeliano[23, 67], e nos leva a

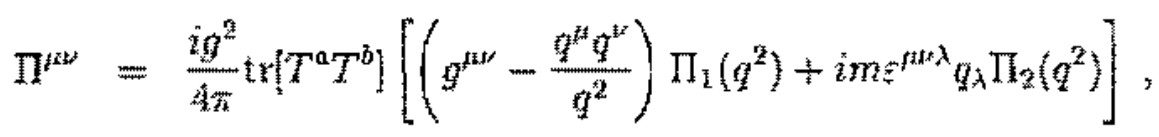

sendo

$$
\begin{aligned}
& \Pi_{1}\left(q^{2}\right)=\int_{0}^{1} d x \frac{2 q^{2} x(1-x)}{\left[m^{2}-q^{2} x(1-x)\right]^{1 / 2}} \approx \frac{q^{2}}{3|m|} \\
& \Pi_{2}\left(q^{2}\right)=\int_{0}^{1} d x \frac{1}{\left[m^{2}-q^{2} x(1-x)\right]^{1 / 2}} \approx \frac{1}{|m|}
\end{aligned}
$$

Escrevendo o tensor de polarizaçăo renormalizado $\Pi_{A}^{\mu p}=\Pi^{\mu \prime \prime}-2 \hat{b} \theta z^{k \nu \lambda} q_{\lambda}$, e escolhendo $\delta \Theta$ de modo a assegurar que nào teremos induģăo do termo de Chern-Simons, isto é. para năo precisarmos redefinir o paramêtro de Chern-Simons, obtemos para pequenos momentos,

$$
\Pi_{R}^{\beta B s}=\frac{i g^{2}}{12 \pi|m|} \operatorname{tr}\left[T^{a} T^{b} \mid\left(q^{2} g^{i \mu \prime}-q^{i t} q^{z}\right)\right.
$$

Os gráficos com duas linhas extemas do canpo de gauge. mas sem campos de matéria, como no caso escalar, nầo däo nenhuma contribuiça para o tensor de polarizaçăo. 


\subsubsection{Correção de Vértice}

Os gráficos que darão contribuição para a corrẹăo de vértice săo mostrados na figura (3.7a-c). Os gráficos (3.7 d-e) são necessários para o cálculo do momento magnético e também contribuirão no espalhamento. O gráfico (3.7a) nos leva a

$$
\Gamma_{a}^{a, g}=\frac{g^{3}}{\Theta}\left[\gamma^{b} T^{a} T_{b}\right] / \frac{d^{3} k}{(2 m)^{3}} \frac{N^{i k}}{\left[(p-k)^{2}-m^{2}+i e\left[\left(p^{f}-k\right)^{2}-m^{2}+i e\left[-k^{2}\right]\right.\right.}
$$

sendo

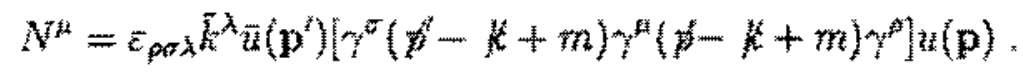

Usando as propriedades das matrizes de Dirac obtemos para a componente 0

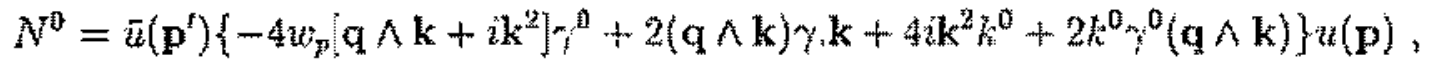

e para a componente $i$

$$
\begin{aligned}
& N^{i}=\bar{u}\left(\mathbf{p}^{i}\right)\left\{2(\mathrm{q} \wedge \mathrm{k})\left(k^{0}-2 \mathrm{p}^{0}\right) \eta^{i}-2 i \mathrm{k}^{2} p^{i}\right. \\
& \left.+\left[2(\mathrm{q} \wedge \mathrm{k}){ }^{0}+2 \mathrm{P} \cdot \mathrm{k}+2 i \mathrm{k}^{2}+2 i k_{\hat{g}}^{2}\right] k^{i}\right\} u(\mathrm{p})
\end{aligned}
$$

Fazendo a expansão para pequenos momentos, usando (3.39), e mantendo termos até ordem $\mathrm{p} / m$. obtemos, como no caso abeliano[67], para a componente 0 de $(3.68)$

$$
\Gamma_{a}^{a .0}=O\left(\eta^{2}\right)
$$

Para a componente $i$ de (3.68), após a integraçào em $k^{0}$,

$$
\Gamma_{a}^{a, i}=-\frac{i g^{3}}{8 \pi^{2} \Theta}\left[T^{b} T^{s} T_{b}\right] \int d^{2} \mathrm{k} \frac{1}{\mathrm{k}^{2}} \frac{w_{p-k}-u_{p^{z}-k}}{2 \mathrm{q} \cdot \mathrm{k}}\left[B^{i}-\frac{A^{i}}{w_{p-k}^{2} w_{p^{j}-k}}\right],
$$

sendo

$$
\begin{aligned}
& A^{i}=-2 w_{p}(\mathrm{q} A \mathrm{k}) \eta^{i}+\left[2(\mathrm{q} A \mathrm{k}) \eta^{\mathrm{n}}+2 i u^{2}+2 \mathrm{P} \cdot \mathbf{K}+2 i \mathbf{k}^{2}\right] k^{i}-2 i \mathbf{k}^{2} P^{i} \\
& B^{i}=2 i k^{i}
\end{aligned}
$$

Fazendo a separaça nos setores $L$ e $H$. obtemos para o setor de baixa energia

$$
\Gamma_{a}^{a, 3}=O\left(\eta^{2}\right)
$$

e para a parte de alta energia

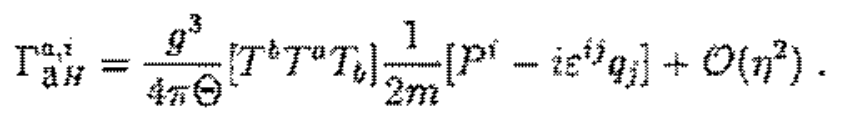




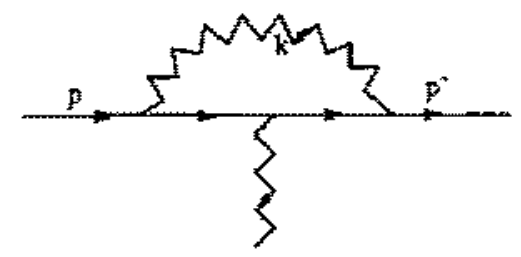

$\mathbf{z}$

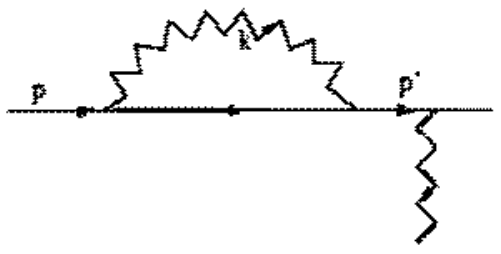

d
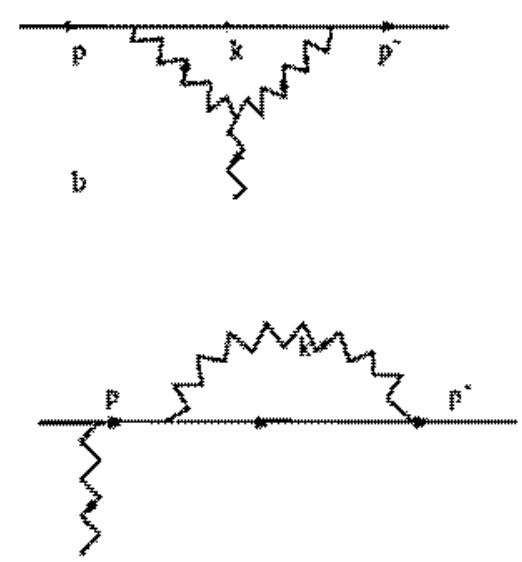

(

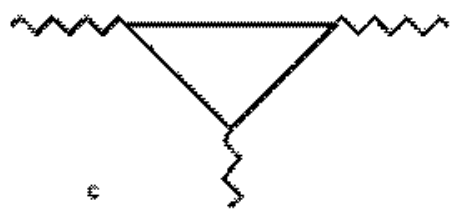

Figura 3.7: Correçino de Vêrotuce.

Como era de se esperar: a contribuf̧̧a vem somente do setor du alta enegia.

O gráfico (3.7c), cuja divergência logaritimica cancelada pela sinetria na integraçă juntamente com o gráfico con quatro linhas externas do campo de gauge e o tensor de polarizaçäo induzem um termo de Maxwell para a Lagrangeana original. Porén eles não darão nenhuma contribuiçäo para o espalhamento até 1-loop. O Gráfico (3.7̄b) ¿ dado por

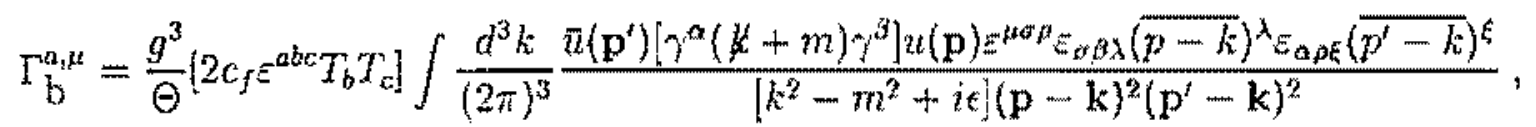

e nos leva a

$$
T_{b}^{a \cdot a}=O\left(\eta^{2}\right)
$$

e para a componente :

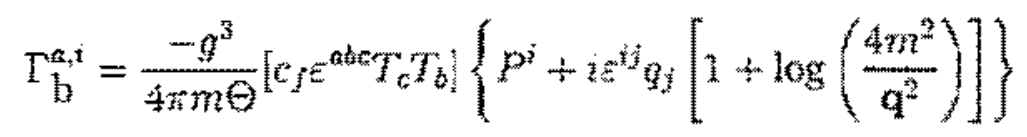

Os gráficos $(3.7 \mathrm{~d}-\mathrm{e})$ são dados por

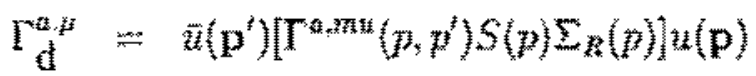

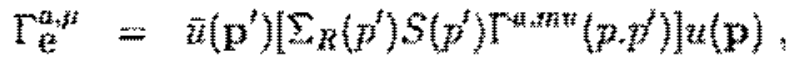


Da equaçăo $(3.52)$ e da relaçäo $\Sigma_{R}=\Sigma+1$ b̆m, temos

$$
\begin{aligned}
& \Gamma_{\mathrm{d}}^{a, 0}=\Gamma_{\mathrm{e}}^{\mathrm{a}, \mathrm{b}}=\mathcal{O}\left(\eta^{2}\right) \\
& \Gamma_{\mathrm{de}}^{a_{\mathrm{e}} i}=\Gamma_{\mathrm{d}}^{a_{i}}+\Gamma_{\mathrm{e}}^{a_{i} i}=-\frac{g^{3}}{4 \pi \Theta}\left[\left(T^{\dot{\phi}} T_{b}\right) T^{a}\right]\left\{\frac{1}{2 m}\left[P^{i}+i \epsilon^{i j} q_{j}\right]\right\} \text {. }
\end{aligned}
$$

Dos grăficos acima, podemos verificar qual a correção para o momento magnético. Ternos que a contribuiçăa da parte local proporcional a $\varepsilon_{i j} q^{j}$ que vem da equaçäo (3.80) cancela exatamente a contribuição extra que vem de (3.77) devido a estrutura do grupo. On seja, usando (3.2) temos que $T^{b} T^{a} T_{b}=T^{a}\left(T^{b} T_{b}\right)-2 c_{f} \varepsilon^{a b c} T_{c} T_{b}$. Assim em (3.77), a contribuição proporcional a $\varepsilon^{\text {abect }} T_{c} T_{b}$ cancela a contribuição que vem de (3.78), enquanto a parte proporcional a $T^{a}\left(T^{\mathrm{b}} T_{b}\right)$ e a parcela de (3.83) nos dào a contribuição para a correçào do momento magnético. Assim o termo que contribui para o monrento magnético fica

$$
\Gamma_{m a g}^{i, a}=\frac{i g^{3}}{4 m \pi \Theta}\left[\left(T^{b} T_{b}\right) T^{a}\right] \varepsilon^{i j} q^{j}
$$

levando a um monento magnético

$$
\mu_{1-i \text { tcop }}^{a}=\frac{i g^{3}}{4 \pi m \Theta}\left[T^{a}\left(T^{b} T_{b}\right)\right],
$$

Podemos ver que a equaçăo acima difere do caso Abelino devido ao fator de grupo, porén no limite Abeliano $(g=e \sqrt{2}$ e $T=i / \sqrt{2})$ recuperamos o resultado da referẽncia [23].

\subsubsection{Espalhamento em 1-Loop}

Nesta seçäo vamos calcular o espalhamento férmion-férmion, até 1-loop, tomando o limite para monentos externos pequenos. Para os momento internos faremos a separaçăo de baixa e alla energia introduzindo 0 corte intermediário $A$, como feito no cáso escalar. No apêndice $\mathrm{D}$ explicitaremos a contas param gráfico. Na figura $(3.8)$ temos os gráficos que contribuem para o espalhamento até 1-loop. A nivel de árvore temos o gráfico $(3.8 a)$,

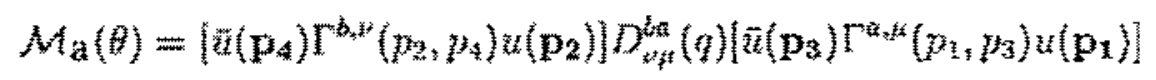

que con o uso da regras de Feymnan e das relaçồ (3.39) a equação atima nos leva, em orden dominante, a

$$
M_{\mathrm{a}}(\theta)=\frac{i g^{2}}{m \theta}\left[T^{2} \otimes T_{\mathrm{a}}\left\{1+i \frac{\sin \theta}{(1-\cos \theta)}\right\} .\right.
$$

O resultado acima é exatamente o resultado obtido na teoria não relativística a nivel de árvore. 

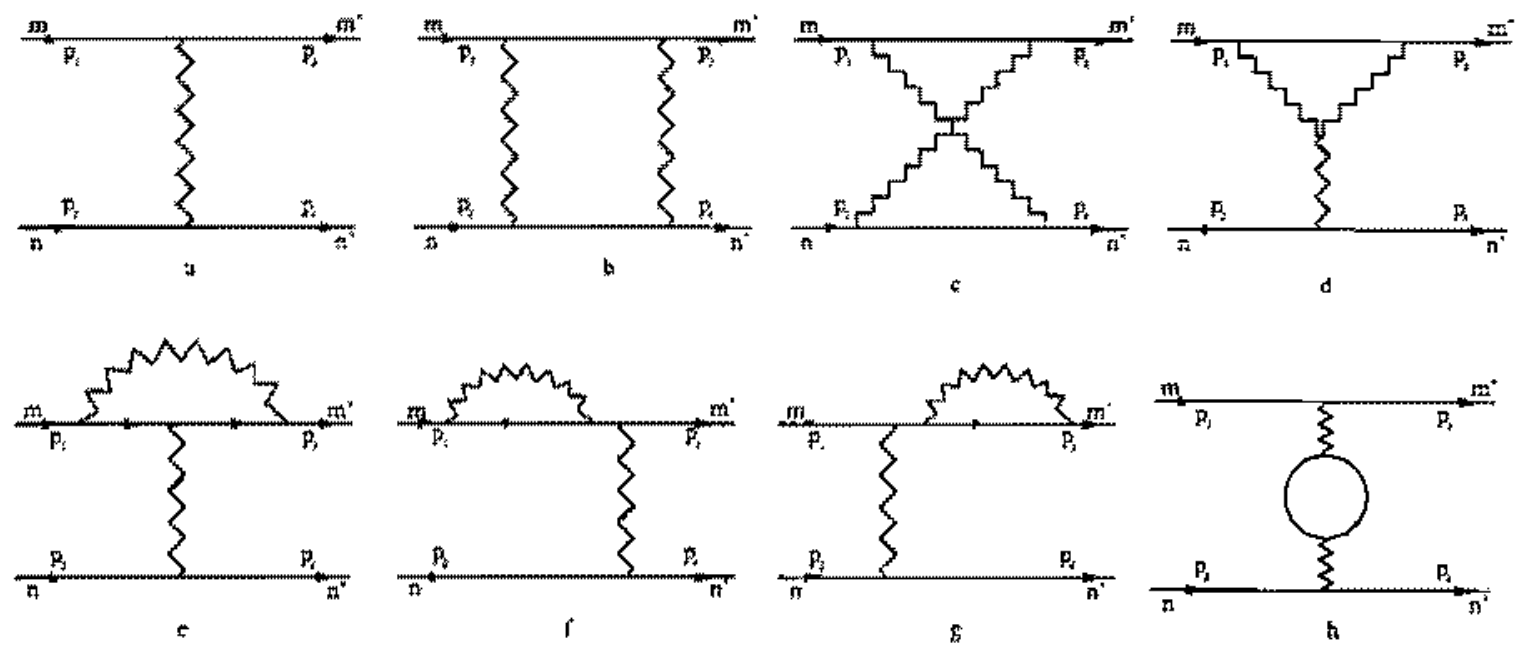

Figura 3.8: Espalhamento Férmian-Fërmion - Teorá Relativistica.

A seguir vamos calcular as correföes de l-loop para o espalhamento. Os gráficos que irăo contribuir nesta orden săo dados na figura $(3.8 \mathrm{~b}-\mathbf{h}) .0$ gráfico (3.8d) não existem na teoria Abeliana, enquanto que us outros aparecen na teoria Abeliana, porém com of fator multiplicativo extra que vern da grupo de simetria interna.

\section{Gráfico Direto}

A amplitude de espalhamento do diagrana (3.8b) é

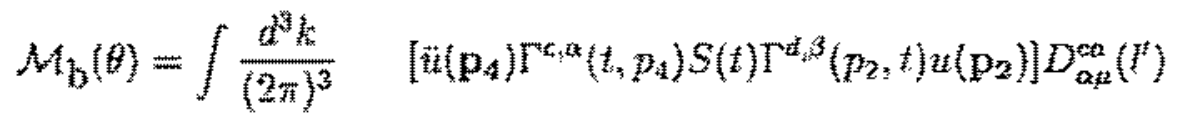

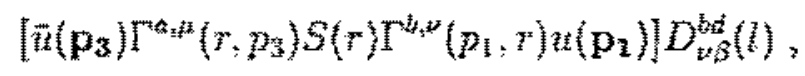

onde $l=\left(k^{0}, \mathrm{k}-\mathrm{p}_{1}\right)_{1}{ }^{\prime}=\left(k^{0}, \mathrm{k}-\mathrm{p}_{3}\right), r=\left(w_{k}+k^{0}, \mathrm{k}\right), e l=\left(w_{p}+k^{0} ;-\mathrm{k}\right)$. Usando o fato que o propagador do campo de matéria pode ser escrito como[n]

$$
S(p)=i\left[\frac{u(\mathrm{p}) \tilde{u}(\mathrm{p})}{z^{0}-w_{p}+i t}+\frac{v(-\mathrm{p}) \underline{u}(-\mathrm{p})}{v^{0}+w_{\mathrm{p}}-i t}\right]
$$

obtemos, após a integraçäo em $k^{0}$ :

$$
\mathcal{M}_{b}(\theta)=\frac{i g^{4}}{2}\left[T^{a} T^{b} \otimes T_{a} T_{b}\right] \int \frac{d^{2} \mathrm{k}}{(2 \pi)^{2}}\left[\frac{T\left(k, p_{1}\right) T^{*}\left(k, p_{3}\right)}{u_{k}-w_{p}}+\frac{H\left(p_{3}, k\right) H^{*}(p, k)}{w_{k}+w_{p}}\right]
$$


sendo

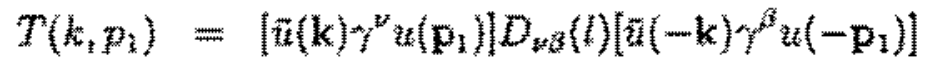

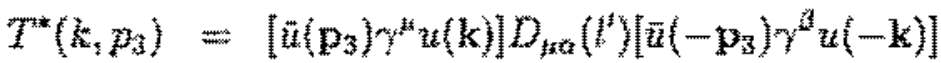

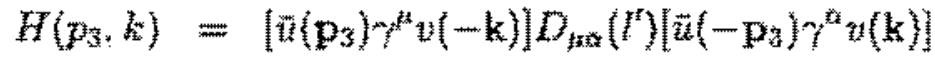

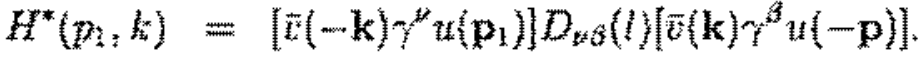

Utilizando as equatocoss $(3.39)$ verifteamos que

$$
\begin{aligned}
& T\left(k_{1}\right)=\frac{2 z}{\ominus(\mathrm{k}-\mathrm{p})^{2}}\left\{\left(\frac{w_{k}+m}{2 w_{k}}\right)\left(\frac{w_{p}+m}{2 w_{p}}\right)\left[1+\frac{k_{-} p_{+}}{\left(w_{k}+m\right)\left(w_{p}+m\right)}\right]\right. \\
& \left.\times\left(\frac{l-p_{+}}{w_{p}+m}-\frac{1+h}{u_{w}+m}\right)\right\} \\
& H\left(p^{\prime}, k\right)=\frac{2 i}{\Theta\left(l^{\prime}\right)^{2}}\left\{\left(\frac{w_{p^{\prime}}+m}{2 u_{p^{\prime}}}\right)\left(\frac{w_{k}+m}{2 w_{k}}\right)\left[\frac{p^{\prime}}{w_{p^{\prime}}+m}-\frac{k_{-}}{w_{k}+m}\right]\right. \\
& \left.\times\left(l_{-m}^{\prime}+y_{+}^{\prime} \frac{p^{\prime} k}{\left(z_{k}+m\right)\left(w_{p^{\prime}}+m\right)}\right)\right\} \text {. }
\end{aligned}
$$

Dividindo a amplitude (3.90) en duas partes, $\mathcal{M}_{b}^{e t}(\theta)$ e $\mathcal{M}_{b}^{p p}(\theta)$, onde

$$
\mathcal{M}_{\mathrm{b}}^{\mathrm{e}}(\theta)=\frac{i g^{4}}{2}\left[T^{a} T^{b} \& T_{n} T_{b}\right] \int \frac{d^{2} \mathrm{k}}{(2 \pi)^{2}}\left[\frac{T\left(k_{1} p_{1}\right) T^{n}\left(k, p_{3}\right)}{w_{k}-w_{p}}\right]
$$

e

$$
\mathcal{M}_{\mathrm{b}}(\theta)=\frac{i g^{4}}{2}\left[T^{a} T^{b} \otimes T_{\mathrm{a}} T_{b}\right] \int \frac{d^{2} \mathrm{k}}{(2 \pi)^{2}}\left[\frac{H\left(p_{3}, k\right) H^{*}(p, k)}{w_{k}+w_{p}}\right]
$$

e fazendo a separapăo no setor de baixa e alta energia, obtenos

$$
\begin{aligned}
& M_{\mathrm{b} L}^{*}(\theta)=\frac{i \eta^{4}}{4 \pi m \Theta^{2}}\left[T^{a} T^{b} \otimes T_{a} T_{b}\right]\left\{\log _{g}\left(\frac{\Lambda^{2}}{\mathrm{q}^{2}}\right)+\mathcal{O}(\eta)\right\} \\
& M_{\mathrm{b} H}(9)=\frac{\eta^{4}}{4 \pi m \Theta^{2}} T^{2} T^{3} \otimes T_{5}\left(\log \left(\frac{2 m^{2}}{n^{2}}\right)+O(\eta)\right\}
\end{aligned}
$$

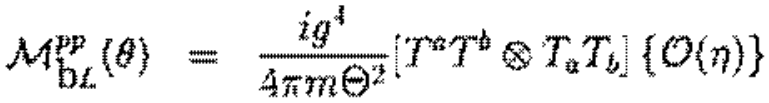

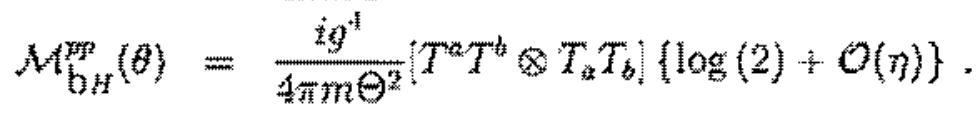

Ou seja, em orden dominarte tamos

$$
\left.M_{\mathrm{b}}(\theta)=\frac{i g^{4}}{4 \pi \mathrm{C}^{2}}\left[T^{2} T^{2} \otimes T_{a} T_{1}\right] \log \left(\frac{m^{2}}{\mathrm{q}^{2}}\right)\right\}
$$




\section{Grafico Cruzado}

Agora varnos calcular o gráfico $(3.8 \mathrm{c})$, cuja amplitude de espalhamento correspondente 6

$$
\begin{aligned}
M_{c}(\theta) & =-\frac{g^{4}}{\Theta^{2}}\left[T^{b} T^{a} \otimes T_{a} T_{b}\right] \int \frac{d^{3} k}{(2 \pi)^{3}} \frac{1}{\left(\mathbf{p}_{3}-\mathbf{k}\right)^{2}\left(\mathbf{p}_{1}-\mathbf{k}\right)^{2}} \\
& \times\left[\frac{N_{c}}{\left[k^{2}-m^{2}+i \epsilon\right]\left[\left(k-p_{2}-p_{1}\right)^{2}-m^{2}+i \epsilon\right]}\right]
\end{aligned}
$$

com

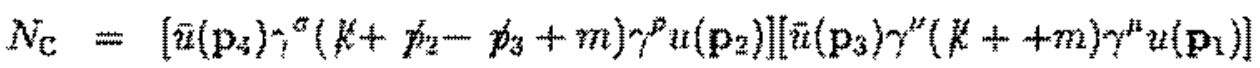

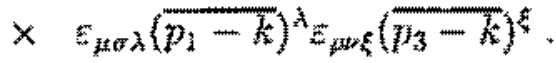

Usando as propriedades da matrizes de Dirac, as relaçöes (3.39), integrando em to a repetindo o mesmo procedinento do corte intemediário obtemos

$$
\begin{aligned}
& \mathcal{M}_{\mathrm{CL}}(\theta)=-\frac{i g^{4}}{\pi m \Theta^{2}}\left[T^{b} T^{a} \otimes T_{a} T_{b}\right]\left\{\frac{1}{2} \log \left(\frac{A^{2}}{\mathrm{q}^{2}}\right)+\mathcal{O}(\eta)\right\} \\
& \mathcal{M}_{\mathrm{CH}}(\theta)=\frac{i g^{4}}{2 \pi m \Theta^{2}}\left[T^{b} T^{a} \otimes T_{a} T_{b}\right\}\left\{1+\frac{1}{2} \log \left(\frac{A^{2}}{4 m^{2}}\right)+\mathcal{O}(\eta)\right\} .
\end{aligned}
$$

Isto é

$$
\mathcal{M c}_{\mathrm{c}}(\theta)=\frac{i g^{4}}{2 \pi m \Theta^{2}}\left[T^{\mathrm{h}} T^{2} \otimes T_{a} T_{t}\right]\left\{1+\frac{1}{2} \log \left(\frac{\mathrm{q}^{2}}{4 m^{2}}\right)+\mathcal{O}(\eta)\right\} .
$$

Observemos que o fator de grupo $\left[T^{b} T^{a} \otimes T_{a} T_{b}\right]=\left[T^{b} T^{a} \otimes T_{b} T_{a}\right]-2 c_{f} \tilde{c}^{a c d} T_{a} T_{a} \otimes T_{d}$

\section{Gráfico com Três Gluons}

O gráfico que contếm o vértice com trếa gluons, $(3,8 d)$ é dado analiticamente por ${ }^{2}$

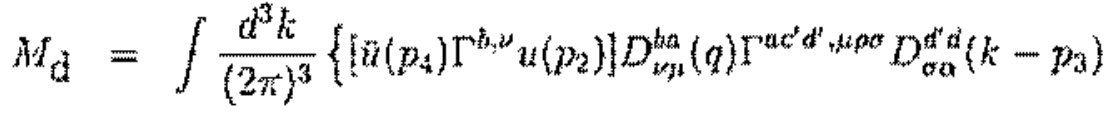

$$
\begin{aligned}
& \left.\times D_{\xi \beta}^{c}\left(k-p_{1}\right)\left[u\left(p_{3}\right) \Gamma^{d, a} S(h) \Gamma^{c, a} u\left(p_{1}\right)\right]\right\} \\
& =\frac{i(-g)^{3}}{(\Theta)^{3}}\left[-2 \operatorname{lig} g \Theta z^{n c t} T_{a} \otimes T_{d} T_{a}\right] \int \frac{d^{3} k}{(2 \pi)^{3}\left[k^{2}-m^{2}+i \varepsilon\right]\left(\mathrm{k}-\mathrm{p}_{1}\right)^{2}\left(\mathrm{k}-\mathrm{p}_{3}\right)^{2} \mathrm{q}^{2}}
\end{aligned}
$$

\footnotetext{
"vẹja apênüice D
} 
com

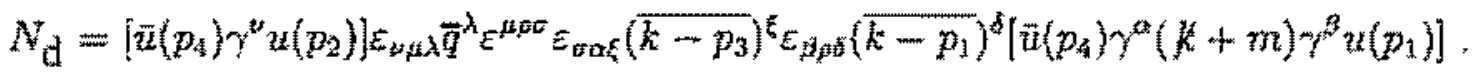

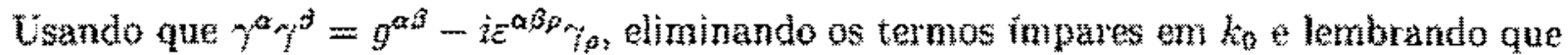
$z_{0 ;} a^{2} b^{\prime}=\mathbf{a} A b_{3}$ podemos escrever até orden $\eta$

$$
N_{d}=\left[q \wedge k-p_{1} \wedge p_{3}\right]\left[22\left(k^{2}+p^{2}\right]-2[q \wedge k+p \cdot k]\right.
$$

Substituindo na equaça $(3.108)$ e integrando em to na parta angular obtemos

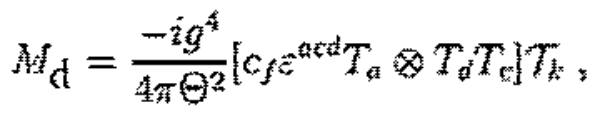

$\operatorname{com}$

$$
\begin{aligned}
T_{k} & =\int_{0}^{A_{\theta}^{2}} \frac{d \mathbf{k}^{2}}{\mathrm{q}^{2} w_{k}}\left\{(1-\cos \theta)+i \sin \theta\left[1-S g\left(\mathrm{k}^{2}-\mathrm{p}^{2}\right)\right]\right. \\
& \left.+S_{g}\left(\mathrm{k}^{2}-\mathrm{p}^{2}\right)\left[2 \mathrm{p}^{2}(1-\cos \theta) \frac{\mathrm{k}^{2}+\mathrm{p}^{2}}{R}\right]\right\}
\end{aligned}
$$

onde $S g\left(k^{2}-p^{2}\right)$ é sinal de $\left(k^{2}-p^{2}\right)$ e $R=\left(k^{4}+p^{4}-2 p^{2} k^{2} \cos \theta\right)$.

Vamos separar a integral em $\mathrm{k}^{2}$ na parte de baixa energia (L) e alta energia (H). Na parte de baixa energia fazemos uma expansăo em $1 /$ th e ma parte de alta fazemos uma expansäo em torno de $\mathrm{p} \approx 0$. Assim para o setor de baixa energia

$$
\begin{aligned}
N_{\mathrm{d}}^{t} & =\frac{-i g^{4}}{4 \pi m \Theta^{2}}\left[c _ { f } \varepsilon ^ { a c d } T _ { \mathrm { a } } \otimes T _ { d } T _ { m } \left\{\left(1+i \frac{\sin \theta}{1-\cos \theta}\right.\right.\right. \\
& \left.+\left[\log \left(\frac{A^{2}}{\mathrm{q}^{2}}\right)-\frac{A^{2}}{2 m^{2}}-(1+2 \cos \theta) \frac{\mathrm{p}^{2}}{A^{2}}\right]\right\}
\end{aligned}
$$

e para o setor de alta energia

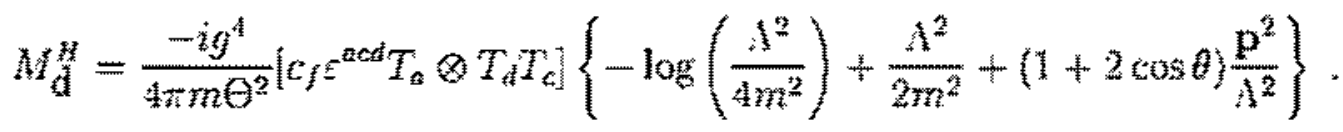

Somando a contribuigão das equaf̧os $(3.113)$ e $(3.114)$ obtemos

$$
M_{\mathrm{d}}=\frac{-i y^{4}}{4 \pi m \theta^{2}}\left[c_{f} \varepsilon^{a c h} T_{\mathfrak{a}} \otimes T_{d} T_{c}\right]\left\{1+i \frac{\sin \theta}{1-\cos \theta}+\log \left(\frac{4 m^{2}}{a^{2}}\right)\right\}
$$




\section{Correções Radiativas}

Consideremos agora as correföes radiativas para os propagadores e vértice (3.7a). As contribuiç̧ôes que vem da inclusầ da correçăo de vértice e auto-energia do férmion são representadas nas figuras (3.8e-g). Utilizando as equaçöes $(3.77)$ e $(3.83)$ obtemos

$$
\begin{aligned}
\mathcal{M}_{\mathrm{e}-\mathrm{g}}(\theta)= & -\frac{i g^{4}}{4 \pi m \Theta^{2}}\left[T^{a} \otimes\left(T^{b} T_{b}\right) T_{a}\right]\{1\} \\
& +\frac{i g^{4}}{4 \pi m \theta^{2}}\left[c_{f} z^{\mathrm{abc}} T_{0} \otimes T_{c} T_{b}\right]\left\{1+i \frac{\sin \theta}{(1-\cos \theta)}\right\} .
\end{aligned}
$$

Lembrando que para os gráficos (3.8d-g) temos um fator 2 devido ao gráfico invertido.

Por último temos a incorporaça do tensor de polarizaçäo, representado na figura $(3.8 \mathrm{~h})$,

$$
\mathcal{M}_{\mathrm{h}}(\theta)=\frac{i g^{4}}{24 \pi m \Theta^{2}}\left[T^{n} \otimes T_{a}\right] .
$$

A soma das contribuiçöes, equaçöes $(3.102),(3.107),(3.115),(3.116)$ e $(3.117)$ leva a

$$
\begin{aligned}
\mathcal{M}_{1-\text { - oop }}(\theta) & =-\frac{i g^{d}}{4 \pi m \Theta^{2}}\left\{\left[T^{\alpha} \otimes\left(T^{b} T_{b}\right) T_{b}\right](2)-\left[T^{b} T^{a} \otimes T_{b} T_{a}\right](2)\right. \\
& \left.+\left[c_{l} \varepsilon^{a c d} T_{a} \otimes T_{d} T_{d}\right](4)-\left[T^{a} \otimes T_{a}\right](1 / 6)\right\} .
\end{aligned}
$$

que com o uso das propriedades das matrizes $T$ pode ser escrito na forma

$$
M_{1-i \text { oop }}(\theta)=\frac{i g^{4}}{4 \pi m \theta^{2}}\left\{\frac{3}{8}[J \otimes I]+\frac{2}{3}\left[T^{a} \otimes T_{n}\right]\right\} .
$$

\subsection{Análise}

Observernos inicialmente que os dois primeinos termos, indusive ofator dependente do ângulo de espalhamento, da equaçăo (3.115), säo cancelados pela segunda parte de (3.116). No entanto, no primeiro caso esta contribuiça ven do setor de baixa energia $(L)$. enquanto no segundo caso vem do setor de alta energia $(H)$. A segunda parcela em (3.115) vern exclusivamente do gráfico (3.8e). Isto tambẻm ncontece no cálculo das correçös radiativas da inserçăo de vértice e auto-energia, primeira parcela da equaçăo (3.116). Esta primeira parcela tem contribuigho dos grâficos $(3.8 \mathrm{e}-\mathrm{g})$. Tambén verificamos que os termos que dependem de $\log \left(A^{2}\right)$, no setor $L$, säo exatamente cancelados pela contribuiçăo do setor $H$. Isto $\hat{e}$, a parte de alta energia fornece um contra-temo natural como acontece 
no caso escalar. Porém, diferentemente do caso escalar, os gráficos do espalhamento nāo relativístico são finitos e invariantes de escala. No caso fermiônico o termo de interação de Pauli faz o papel do termo de contato que aparecia no caso de spin 0 .

A contribuição para o espalhamento dada pela equação (3.119), são correçōes ao espalhamento e não aparecem na teoria não relativistica. Esta contribuição, como vemos, aparece já em ordem dominante. Um extensão possível ao espalhamento $A B$ nāo-Abeliano de spin $1 / 2$ seria extendermos os cálculos, como foi feito no caso escalar, para obtermos as correçôes sub-dominantes. No entanto isto implica num trabalho mais extenso do que no caso escalar, e fica cono uma possibilidade futura. 


\section{Capítulo 4}

\section{Partícula Massiva de Spin 1 Interagindo com Chern-Simons}

\subsection{Introdução}

Como vimos nos capitulos anteriores, o espalhamento de uma particula carregada por um campo de CS tem se mostrado un problema interessante. Neste capitulo gostarianos de estender a discusäo para o caso de spin 1. Lma análise usando a mecanica quâtica tem sido feito nas referencias [20] e [21]. No primeiro cxso os autores trabalharam com o modelo de Proca carregado ençuanto no segundo caso fó empregado modelo de YangMills linearizado. No caso de um estudo perturbativo atraves da teoria de campos, nesmo para o caso Abeliano, o problema apresenta dificuldades adicionais devido a certas caracteristiscas que surgen na fomulaçâ de um modelo descrevendo um méson vetorial massivo.

O problema inicial reside no fato que temos mais do que uma formulação para tratarmos uma partícula carregada de spin 1, como jú ressaltado no capitulo 1. Como foi visto, para descrevernos um campo vetorial massivo liyta temos formulaçoes equivalentss, isto é a modelo AD equivalente ao modelo do MCSLOI. Por ontro lado o modelo AD[9] é uma topecte de "ratz quadrada" do modelo de Procal65]. Ko entanto, quando estes modelos esta acoplados a outro campo dinamico, tomo por exemplo o campo de Dirac, temos que ter acoplamentos diferentes para que a identificaça seja possivel.

Neste capitulo ramos andisar especificamente os modelos de MCS e Proca carrega dos, quando acoplados con um tampo de CS. Veremos, que devido a forma dos vertices e propagadores que aparecem en ambos os modelos, uma análise completa até 1-loop se torna inviatval. Isto e devido ao numero de termos e parâmetros de Feyman que anarecen no cálculo das integrais. Temos tambén que no caso do modelo de Proca, a forma do propagador do campo de gauge carregado, pode levar a um comportamento rum no ultravioleta. Isto näo acontece no caso do modelo de MCS: mas neste caso a dificuldade 
reside no numero de parämetros de Feynman nas integrais de 1-loop. Entretanto, mesmo nầo tendo sido finalizado os calculos ate 1-loop, nós obtemos alguns resultados interessantes ja a nítul de arvore. Alếm disto, faremos uma análise das correços radiativas para o modelo de Proca minimamente acopiado com $\mathrm{CS}$.

O modelo de Proca carregado com massa $M$, destrito pela densidade de Lagrangeana

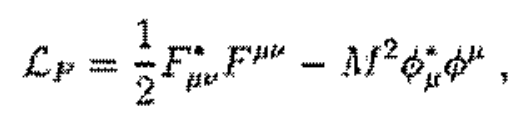

onde $F^{* t}=\partial_{\mu} \psi_{w}-\partial_{w} \phi_{p}$. O nodelo Maxwell-Chern-Simons (MCS) carregado é dado por

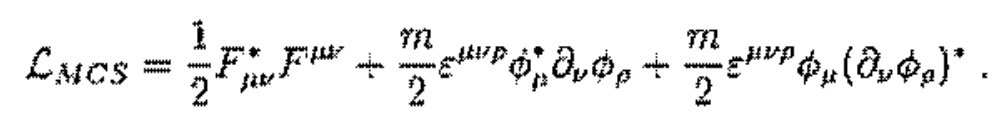

Como veremos, o primeiro descreve dois modos massivos de massa $M$ e spin \pm 1 , enquanto o que o segundo apenas um modo massivo de massa $m$ e spin $\frac{m}{|m|}$.

A seguin vamos introduzir o modelo de Proca, determinar os vetores de polarização, as regras de Fesnman e a amplitude de espathamento a nivel de arvore. Posteriomente repetiremos para o caso de MCS. Na seçäo 2.3 vamos implementar a correçäo de 1-loop para o modelo de Proca.

\subsection{Vetores de Polarização e Regras de Feynman}

\subsubsection{Modelo de Proca}

Vamos primeiramente tomar a Lagrangeana de Proca dada por (4.1). Para uso futuro vamos estudar alguns aspectos do modelo livre. Para isto, vamos expandir $\phi^{\mu}$ em ondas planas

$$
\phi^{* x}=\frac{1}{(2 \pi)} \int \frac{d^{2} p}{2 w_{p}} \sum_{\lambda} f_{\lambda}(p)\left[a_{\lambda}(p) e^{-i p x}+b_{\lambda}^{\dagger}(p) e^{i p x t}\right]
$$

onde $a, b, a^{*}$ e $b^{\dagger}$ sä̊ os operadores de criaçäo e destruif̧ăo de particulas e antiparticulas, e,

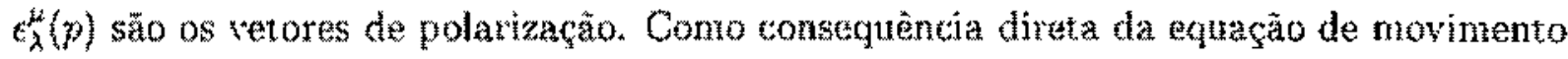
obtenos que of é transversal. Isso reduz a número de componentes de três para dois.

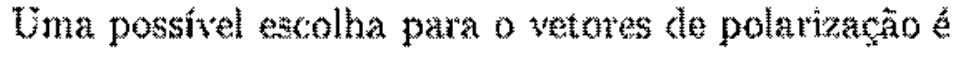

\footnotetext{
${ }^{1}$ Poderiamns ter dois karnpos de NCS com massas topológicas de sinais diferentes para descrever os dois modos de spiz: 1 .
} 


$$
\epsilon_{1}^{\mu}(p)=\left\langle 0, \varepsilon^{i j} \hat{p}_{j}\right) \quad \epsilon_{2}^{\mu}(p)=\left(\frac{|\mathrm{p}|}{M}, \frac{w_{p}}{M} \hat{p}^{i}\right),
$$

$\operatorname{com} w_{p}=\sqrt{M^{2}+|\mathbf{p}|^{2}}$ e $\hat{p}=\frac{\mathbf{p}}{|\mathbf{p}|}$.

Por outro lado, se considerarmos o operador de momento angular

$$
J=\int d^{2} x \varepsilon_{i j} x^{i}: T^{0 j}:,
$$

onde $T^{\mu \nu}$ é o tensor de energia momento simétrico

$$
T^{\mu \nu}=F^{\dagger \mu \rho} F_{\rho}{ }^{\nu}+F^{\mu \rho} F_{\rho}^{\dagger}{ }^{\nu}-g^{\mu \nu} \mathcal{L} .
$$

obteremos

$$
\begin{aligned}
J & =\int d^{2} x\left\{\varepsilon_{i j} x^{i}:\left[\left(\partial_{\mu} \partial^{\mu} \phi^{\dagger 0}\right) \phi^{j}+\left\langle\partial_{\mu} \partial^{\mu} \phi^{0}\right) \phi^{\dagger j}\right]:\right. \\
& +\varepsilon_{i j} x^{i}:\left[\phi^{\dagger 0} \partial^{j} \partial^{0} \phi+\phi^{0} \partial^{j} \partial^{0} \phi^{\dagger}-\partial^{0} \phi^{\dagger k} \partial^{j} \phi_{k}-\partial^{0} \phi^{k} \partial^{j} \phi_{k}^{\dagger}\right]: \\
& \left.+\varepsilon_{i j}:\left[-\partial^{0} \phi^{\dagger i} \phi^{j}-\partial^{0} \phi^{i} \phi^{\dagger j}\right]:\right\} .
\end{aligned}
$$

Usando $\phi^{\mu}(x)$ dado na equaçào (4.3) podemos separar $J$ nas partes $J^{++}, J^{+-}, J^{-+}$e $J^{--}$; onde $+\mathrm{e}-$ referem-se as partes de criação e destruiçāo de cada fator. Por exemplo o termo $J^{+-} \dot{e}$

$$
\begin{aligned}
J^{+-} & =\int d^{2} x\left\{\varepsilon_{i j} x^{i}:\left[\left(\partial_{\mu} \partial^{\mu} \phi_{(+)}^{\dagger 0}\right) \phi_{(-)}^{j}+a . h .\right]:-\varepsilon_{i j}:\left[\partial^{0} \phi_{(+)}^{\dagger i} \phi_{(-)}^{j}+a . h .\right]:\right. \\
& \left.+\varepsilon_{i j} x^{i}:\left[\phi_{(+)}^{\dagger 0} \partial^{j} \partial^{0} \phi_{(-)}-\partial^{0} \phi_{(+)}^{\dagger k} \partial^{j} \phi_{k(-)}+a . h .\right]:\right\},
\end{aligned}
$$

onde a.h. designa o adjunto hermitiano. Escrevendo explicitamente em termos dos operadores $a, a^{\dagger}, b$ e $b^{\dagger}$, e integrando em $x$ podemos decompor $J^{+-}$em três partes

$$
J^{+-}=J_{L}^{+-}+J_{S}^{+-}+J_{p_{u} p^{\prime \prime}}^{+-}
$$

sendo

$$
\begin{array}{r}
J_{L}^{+-}=\quad i \int \frac{d^{2} p}{4 w_{p}} \sum_{\lambda, \lambda^{\prime}=1}^{2} \varepsilon_{i j} p^{j}\left[\epsilon^{\mu}\left(p, \lambda^{\prime}\right) a_{\lambda^{\prime}}^{\dagger}(p) \frac{\partial}{\partial p^{i}}\left(\epsilon_{\mu}(p, \lambda) a_{\lambda}(p)\right)\right. \\
\left.+\epsilon^{\mu}\left(p, \lambda^{\prime}\right) b_{\lambda^{\prime}}^{\dagger}(p) \frac{\partial}{\partial p^{i}}\left(\epsilon_{\mu}(p, \lambda) b_{\lambda}(p)\right)\right] \\
J_{S}^{+--}=-i \int \frac{d^{2} p}{4 w_{p}} \sum_{\lambda, \lambda^{\prime}=1}^{2} \hat{\epsilon}_{i j} \epsilon^{i}\left(p, \lambda^{\prime}\right) \epsilon^{j}(p, \lambda)\left[a_{\lambda^{\prime}}^{\dagger}(p) a_{\lambda}(p)+b_{\lambda^{\prime}}^{\dagger}(p) b_{\lambda}(p)\right]
\end{array}
$$




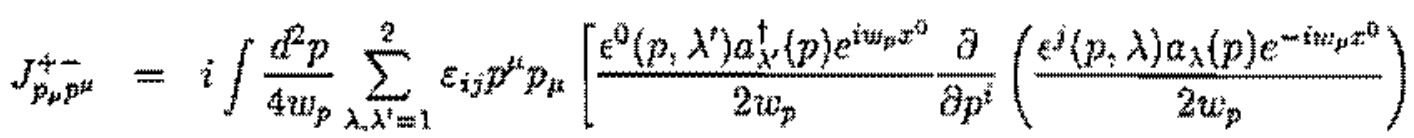

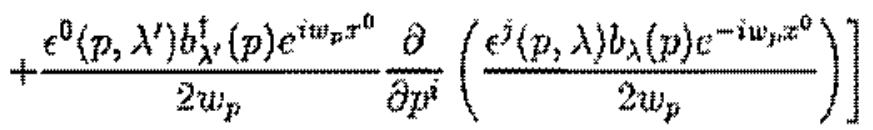

Podemos fazer o mesno para os outros termos. Vemos que $J^{++}$e $J^{-m}$ săo nulos. Para obtermos a contribulçäo do spin, vamos calcular o operador de momento angular no estado de repouso, $(J \mid \mathrm{p}=0 . s>)$. Neste caso o termo $J_{L}$ é nulo e o termo $J_{p_{k}}^{+\cdots}$ se cancela com $J_{p_{p} p^{\mu}}^{++}$. Assim a parte que contribui para o spin é dada por

$$
J_{S}=J_{S}^{+-}+J_{S}^{++}=-i \int \frac{d^{2} p}{2 u_{p}^{\prime}} \sum_{\lambda \lambda^{\prime}=1}^{2} \epsilon_{\lambda^{\prime}}(p) \wedge \epsilon_{\lambda}(p)\left[a_{\lambda^{\prime}}^{\dagger}(p) a_{\lambda}(p)+b_{\lambda^{\prime}}^{\dagger}(p) b_{\lambda}(p)\right] .
$$

Usando os vetores (4.4), podemos escrever, para o setor de particulas,

$$
J_{s}=i \int \frac{d^{2} p}{2 w_{p}}\left[a_{2}^{\dagger}(p) a_{1}(p)-a_{1}^{\dagger}(p) a_{2}(p)\right]
$$

ou seja, os estados

$$
\left|p=0, \lambda=1>=a_{1}^{\dagger}(0)\right| 0>\quad \theta \quad\left|p=0, \lambda=2>=a_{2}^{\dagger}(0)\right| 0>
$$

não săo automestados do operador $J$. Os antomestados do operador $I$ com automalores \pm 1 são dados por

$$
\left.\left|p=0, s= \pm 1>=\frac{a_{1}(0) \pm a_{2}^{\dagger}(0)}{\sqrt{2}}\right| 0\right\rangle
$$

Isto $\dot{e}_{\mathrm{q}}$

$$
J \mid p=0, s= \pm 1> \pm= \pm=0, s= \pm 1>
$$

Assim, para construir un watad de spin \pm 1 , temos que tomar una combinaçä́ dos estados $(4.10)$.

Vamos agora fazer o acoplamento minimal do motelo de Proca com o campo de CS. Deste modo (4.1) fica sendo

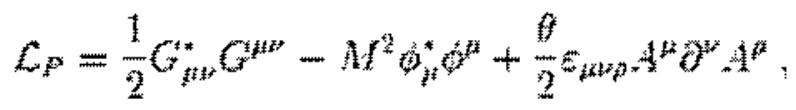

com

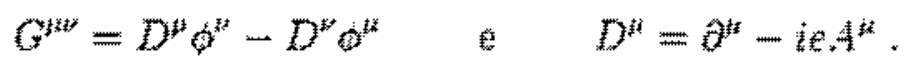




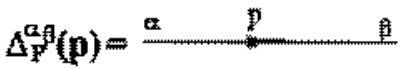

FIg. 1.a

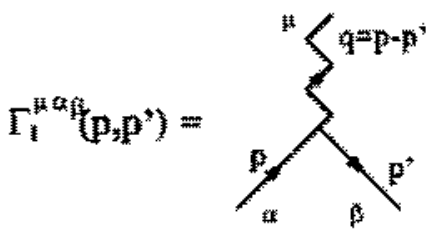

FIg. 1 .

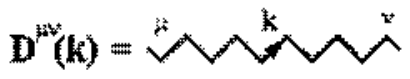

Fig. 1.b

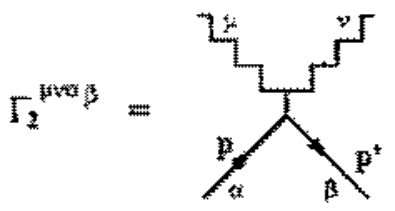

W.

Figura 4.1: Regras de Feynman.

As regras de Feynman no Calibre de Landau para a Lagrangeana (4.18), representadas gráficamente na figura (4.1) são:

Propagador para o campo de matéria

$$
\Delta_{p}^{\mathrm{a}, a}=\frac{-i}{p^{2}-M^{2}}\left[g^{\mathrm{a} 3}-\frac{p^{\mathrm{a}} p^{3}}{M^{2}}\right]
$$

Propagador para o campo de Chern-Simons

$$
D_{k \mu \alpha}(k)=-\frac{1}{\theta} \varepsilon_{\mu \nu \rho} \frac{k^{\rho}}{k^{2}}
$$

Vértices de Interaçāo

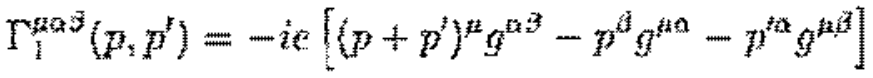

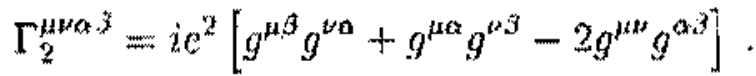

A partir de (4.22) é fácil verificar que

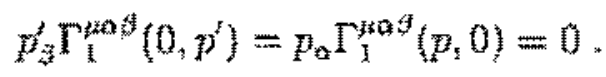

Esta propriedade será útil para a discusăo do comportamento ultravioleta das funçôes de Green.

Neste ponto já podemos obter a amplitude de espalhamento a nivel de árvore. $O$ gráfico que contribui para o amplitude de espalhamento de duas particulas entrando com 


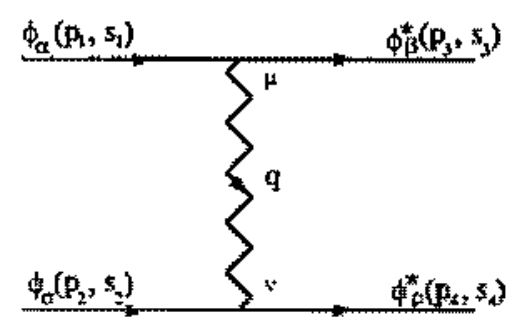

Fig. 2

Figura 4.2: Espahamento 0-Loop.

momentos $p_{1}, p_{2}$ e spins $s_{1}, s_{2}$ e saindo com momentos $p_{3}, p_{4}$ e spins $s_{3}, s_{4}$, é representado na figura (4.2). Analiticamente temos

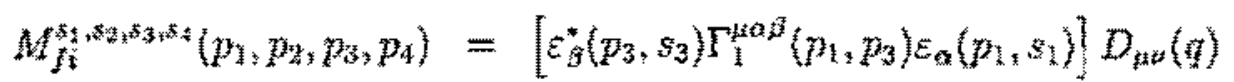

$$
\begin{aligned}
& {\left[\varepsilon_{p}^{*}\left(p_{4}, s_{4}\right) \Gamma_{1}^{\mu \alpha \rho}\left(p_{2}, p_{4}\right) \varepsilon_{2}\left(p_{2}, s_{2}\right)\right]+\left(p_{3} \leftrightarrow p_{4}, s_{3} \leftrightarrow s_{4}\right) \text {; }}
\end{aligned}
$$

onde

$$
\varepsilon_{\alpha}(p, s)=\frac{\epsilon_{1}^{\alpha}(p)+i s \epsilon_{2}^{\alpha}(p)}{\sqrt{2}}
$$

com $\epsilon_{1}^{\alpha}(p)$ e $e_{2}^{\alpha}(p)$ sendo as polarizacoòs dadas por $(4.4)$ e $q=p_{1}-p_{3}=p_{4}-p_{2}$ o momento transferido.

No centro de massa as partículas entrando e saindo tem momentos $p_{1}=\left(w_{p}, \mathbf{p}\right)$, $p_{2}=\left(w_{p},-\mathbf{p}\right), p_{3}=\left(w_{p}, \mathbf{p}^{\prime}\right)$ e $p_{4}=\left(w_{p},-\mathbf{p}^{\prime}\right)$, onde $|\mathbf{p}|=\left|\mathbf{p}^{\prime}\right|$ e $w_{p}=\sqrt{M^{2}+\mathbf{p}^{2}}$. Assim, usando $(4.4),(4.26)$ e as regras de Feynman (4.21) e (4.22), obtemos com a ajuda das relaçöes ${ }^{2}$

$$
\begin{aligned}
& \varepsilon_{n}(p, s) *^{* \mu}\left(p^{\prime}, s^{\prime}\right)=\frac{1}{2}\left[-\left(1+s s^{\prime}\right) \cos \alpha+i \frac{w_{p}}{M}\left(s+s^{t}\right) \sin \alpha+\frac{\mathrm{p}^{2}}{M^{2}}\right], \\
& p_{p} E^{* H}\left(p^{\prime}, s^{t}\right)=\frac{|p|}{\sqrt{2}}\left[-i s^{i} \frac{w_{p}}{M f}(1-\cos \alpha)+\sin \alpha\right]= \\
& \mu_{B} \varepsilon^{*}(p, s)=\frac{|\mathrm{p}|}{\sqrt{2}}\left[s \frac{w_{p}}{M}(1-\cos \alpha)-\sin \alpha\right] \text {, }
\end{aligned}
$$

que, em ordem dominante em $\mathrm{p} / M$, a amplitude de espalhamento fica

$$
\begin{aligned}
M_{P}^{s_{1}=1, s_{2} z_{1}, s_{3}, s_{4}}\left(p_{1}, p_{3}, p_{3}, p_{4}\right)= & \frac{i e^{2} M}{\theta}(\cos \alpha-i \sin \alpha) \\
& \left\{\left(1+s_{3}\right)\left(1+s_{1}\right)\left[1+\frac{i \sin \alpha}{1-\cos \alpha}\right]\right\} .
\end{aligned}
$$

\footnotetext{
${ }^{2}$ Onde temos que $\hat{\mathrm{p}} \cdot \hat{\mathrm{p}}^{\prime}=\cos \alpha \hat{\mathrm{p}} \wedge \hat{\mathrm{p}}^{\prime}=\sin \alpha$. De $(4,4)$ obremos tambern que $\varepsilon_{1}(p)+\hat{\epsilon}_{1}\left(p^{\prime}\right)=$ $E_{i j} \hat{p}_{j} \epsilon_{1}^{i}\left(p^{t}\right)=\varepsilon_{i j} \hat{p}_{j}^{t} \epsilon_{1}^{i}(p)=\cos a$ e $\hat{\mathrm{p}} \cdot \epsilon_{1}\left(p^{t}\right)=-\hat{\mathrm{p}}^{t} \cdot \epsilon_{1}(p)=-\sin \epsilon$
} 


$$
\begin{aligned}
M_{P}^{s_{1}=-1, s_{2}=-1, s_{3}, s_{4}}\left(p_{1}, p_{2}, p_{3}, p_{4}\right)= & \frac{i e^{2} M}{\theta}(\cos \alpha+i \sin \alpha) \\
& \left\{\left(1-s_{3}\right)\left(1-s_{4}\right)\left[-1+\frac{i \sin \alpha}{1-\cos \alpha}\right]\right\}, \\
M_{P}^{s_{1}=1, s_{2}=-1, s_{3}, s_{4}}\left(p_{1}, p_{2}, p_{3}, p_{4}\right)= & \frac{i e^{2} M}{\theta}\left\{\left(1+s_{3}\right)\left(1-s_{4}\right)\left[\frac{i \sin \alpha}{1-\cos \alpha}\right]\right\}, \\
M_{P}^{s_{1}=-1, s_{2}=1, s_{3}, s_{4}}\left(p_{1}, p_{2}, p_{3}, p_{4}\right)= & \frac{i e^{2} M}{\theta}\left\{\left(1-s_{3}\right)\left(1+s_{4}\right)\left[\frac{i \sin \alpha}{1-\cos \alpha}\right]\right\} .
\end{aligned}
$$

As contribuições não nulas em (4.28)-(4.31) são aquelas onde há conservação de spin em cada vértice. Adicionando o gráfico de troca podemos escrever os resultados acima compactamente como

$$
M_{P}(s, \alpha)=\frac{4 i e^{2} M}{\theta} e^{\frac{-i \text { so }}{2}}[s+2 i \operatorname{cotg}(\alpha)]
$$

sendo $s=s_{1}+s_{2}$ a soma dos spins das partículas entrando.

Para $s=0$, ou seja, quando o spin das partículas entrando forem anti-paralelos, o resultado é aquele obtido para a particula escalar sem o termo de contato extra[29]. Igualmente, (4.32) é equivalente, a menos do fator constante fora do parêntese, ao caso espinorial descrito na referência [30].

\subsubsection{Modelo de Maxwell-Chern-Simons}

Vamos agora considerar o caso do méson vetorial topologicamente massivo, substituindo o termo de massa em (4.18) pela massa topológica,

$$
M^{2} \dot{\phi}_{\mu}^{*} \phi^{\mu} \rightarrow \frac{m}{2} z^{\mu \nu \rho} \phi_{\mu}^{*} \partial_{\nu} \phi_{\rho}+\frac{m}{2} z^{\mu \nu \rho} \phi_{\mu}\left(\partial_{\nu} \phi_{\rho}\right)^{*}
$$

Observemos que o termo de massa topológica nāo está contribuindo no termo de interação. i.e. o acoplamento não é minimal. Nós veremos explicitamente mais adiante qual a implicaçăo de termos o acoplamento minimal no caso do espalhamento. Deste modo o propagador de CS e os vértices de interaşāo permanecem os mesmos do modelo de Proca. Para determinarmos o propagador do campo de matéria, precisamos introduzir um termo de fixação de gauge (por conveniência usaremos o gauge de Landau). Assim obtemos que o propagador do campo o $o^{\prime \prime}$ é dado por

$$
\Delta_{M C S}^{\alpha 3}=\frac{-i}{p^{2}-m^{2}}\left[g^{\alpha 3}-\frac{p^{\alpha} p^{3}}{p^{2}}+i m \varepsilon^{\alpha 3 \rho} \frac{p_{\rho}}{p^{2}}\right] .
$$


Para vermos que agora temos apenas um modo se propagando, vamos, do maneira semelhante como foi feito na referencia [0̈4], obter os vetores de polarização. Para isso vamos usar a equaçăo de movinento

$$
\left[\cdots \partial^{2} g_{B_{H}}-m \varepsilon_{\beta^{3} \alpha} \partial^{\alpha}\right] \phi^{\mu}(x)=0 .
$$

Escrevendo $\phi^{\mu}(x)=e^{k}(k) \exp (i k x) \operatorname{com} k_{\mu} \varepsilon^{\mu}(k)=0$, temos

$$
\Sigma_{3 k^{4}} k^{4}(k)=0
$$

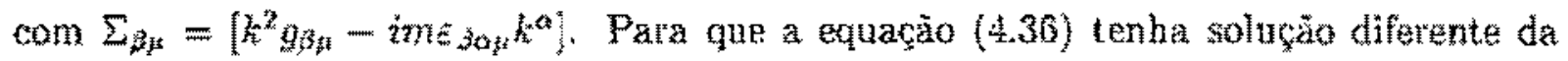
trivial temos que ter

$$
\operatorname{det} \Sigma=\left(k^{2}\right)^{2}\left[k^{2}-m^{2}\right]=0 \text {. }
$$

Para soluça $c$ com $k^{2}=0$. temos de $(4.36)$ que $z^{k}(k)=k^{4 t} \eta(k)$, onde $\eta(k)$ ê uma funçăo arbitrária de $k$. Assim a excitaça sem massa $\dot{e}$ um puro artefato de gauge, visto que numa transformafăo de gauge, $\partial^{k} \rightarrow \phi^{k}+\partial^{k} \eta$.

A outra soluçăo, $k^{2}=m^{2}$, quando substituida em (4.36) para o sistema em tepouso, leva a

$$
\varepsilon^{i t}(0)=\frac{1}{\sqrt{2}}\left(0,1, i \frac{m}{|m|}\right)
$$

Fazendo uma transformaçäo de Lorentz $\epsilon_{\mu}(k)=L_{\mu}{ }^{\prime} \epsilon_{\nu}(0)$ : com

$$
L_{i,}=\left(\begin{array}{c|c}
u_{k} /|m| & k^{(j)} /|m| \\
\hline k^{(i)} /|m|\left|\delta_{i}^{j}-k_{(i)} k^{(j)} /\right| m \mid\left(w_{k}+|m|\right)
\end{array}\right)
$$

nós obtemos

$$
\epsilon_{\mathrm{a}}(h)=\left(\epsilon_{0}(k), \epsilon_{i}(h)\right)
$$

onde

$$
\epsilon_{i}(k)=\frac{\mathrm{k} \cdot(t)}{|m|} \quad \epsilon_{i}(k)=\epsilon_{i}(0)+\frac{\mathrm{k} \cdot \varepsilon(0)}{m\left(w_{p}+m\right)}
$$

Os vetores de polarizaço acinta descrevem um estado com spin $s=\frac{m}{m !}[04]$.

Para compararmos con o restltado (4.32), vamos usar esses vetores de polarização e as regras de Feynman para obtermos amplitude de espalhamento particula-partícula. No centro de massa e até ordem $\mathrm{p} / m$, teremos 


$$
M_{M c s}(s, \alpha)=\frac{4 i e^{2} m}{\theta}[s+2 i \operatorname{cotg}(\alpha)]
$$

onde novamente $s=s_{1}+s_{2}$ é a soma dos spins das partículas entrando e $\alpha$ é o ângulo de espalhamento.

De (4.32) e (4.41) observamos que os resultados diferem por uma fase. No entanto as seções de choque são exatamente as mesmas. Esta fase aparece devido a forma dos vetores de polarizaçāo. No caso de Proca temos

$$
\varepsilon_{\mu}^{*}\left(p^{t}, \lambda\right) \varepsilon^{\mu x}(p, \lambda)=-e^{i k x}+\mathcal{O}\left(\mathrm{p}^{2} / \mu^{2}\right)
$$

enquanto que para MCS temos

$$
\epsilon_{\sharp 1}^{*}\left(p^{\prime}\right) \epsilon^{\mu}(p)=-1+\mathcal{O}\left(\mathrm{p}^{2} / m^{2}\right)
$$

Outro detalhe ta que se tivessemos feito um acoplamento mínimo do campo de $A B$ com o méson topologicantente massivo, isto $e_{2}$ o termo de Chern-Simons do campo de matéria induzindo um termo extra na Lagrangeana de interaçăo, teriamos um fator constante $1 / 4$ multiplieando (4.41).

A parcela constante en $(4.32)$ e $(4.41)$ pode ser interpretada como detorrente de uma interação de contato tipo Pauli entre o momento magnético do méson vetorial e o campo de $A B$. No caso de spins anti-paralelos há um cancelamento deste termo.

\subsection{Correção de 1-Loop}

Se a and̆lise for extendida até 1-loop, esperamos encontrar algumas diferenças entre as duas formulagoes. Isto pode ser visto diretamente do conportamento ultravioleta para o campo de matéria nos dois casos, equaçöes (4.20) e (4.34). No caso do modelo de Proca o termo $p^{k} p^{\nu} / M^{2}$ induz um comportamento ruim no ultravioleta. Ko entanto, quando o momento da linha interna do propagador de matéxia é contraído eom o vértice, a ordem da divergêneia ${ }^{2}$ abaixa, levando a um conportamento equivalente para os dois termos do propagador de matéria. Esta caracteristica será explicitadà mais adiante no cálculo da auto-energia para o campo de matérid. Com isso o grau de divergência superficial efetivo pode ser escrito como

$$
d(\eta)=3-N_{A}-\frac{1}{2} N_{\phi}
$$

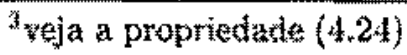




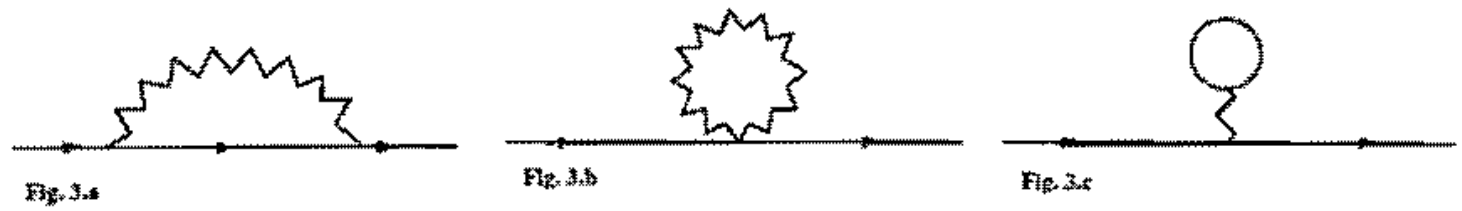

Figura 4.3: Auto-Energia do Méssn Vetorial.

onde $N_{A}$ e $N_{\phi}$ sào os números de linhas externas do campo de Chern-Simons e do campo de matéria. Temos assim que a teoria é renormalizável. Por outro lado se incorporarmos à Lagrangeana inicial os contra-termos induzidos pelas correçōes radiativas a relação (4.44) näo será mais valida. Isto se deve ao fato que as correchess para as funçoes de quatro e seis pontos do campo $\phi$ induzirão vértices não renormalizáveis, e o termo $p^{\mu} p^{\nu} / M^{z z}$ produzirá divergências nos gráficos contento estes novos tipos de vértíces. Se usarmos a regularização dimensional, todos os contra-termos serăo finitos ${ }^{4}$, e podemos tratar como uma teoria efetiva, pois os termos dos vértices induzidos säo de ordern sub dominantes na constante de acoplamento $(e)$. Esse problema năo aparece na formulağăo de massa topológica, descrita pela Lagrangeana (4.2). No entanto, devido a forma do propagador de matéria, os cálculos em 1-loop se tornam mais complicados do que no caso do nodelo de Proca. Assim nas seçöes seguintes vamos nos restringir na anâlise do modelo de Proca minimamente acoplado com o campo de CS.

Da equaçäo (4.44) vemos que os gráficos com $N_{A}=0$ e $N_{\phi}=6$ e com $N_{A}=2$ e $N_{\phi}=2$ apresentam divergencias logaritimicas, mas tomo o espaço de fase é impar, o terno divergente é cancelado por simetria na integraçato. En seguida analisaremos as correçōes radiativas vindas dos outros gráficos que apresentam divergências. Nestes gráficos usaremos a regularização dimensional para efetuarmos a integraçằ nos momentos internos.

\subsubsection{Auto-Energia}

A auto-energia para o campo de matéria é dada en 1-loop pelos gráficos da figura (4.3). Os gráficos (4.3b) e (4.3c) são nulos. O gráfico (4.3a) é

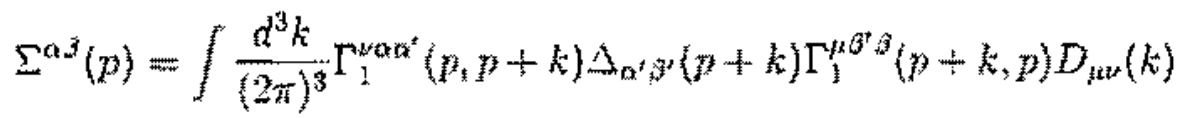

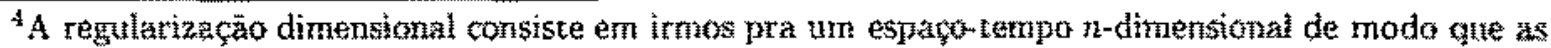
integraç̋es nos "loops" fiquern fnitas. As inregrais de Feynman săo defindas como funçoes analiticas neste espaço e os infinitos aparecem na forma $1 / \epsilon$. onde $\varepsilon=D-\pi$ : guando $n \rightarrow D$. No casso te $D=3$ existe uma pecullaridade devido â fato dos pólos gerados seren na forma de hançôes gamas, $\Gamma\left(-\frac{1}{2}-n / 2\right)$,

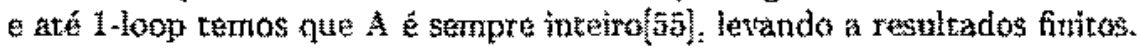


Aqui podemos ver explicitamente como o grau de divergência diminui. Realmente, no produto

$$
\begin{aligned}
& \Gamma_{1}^{\nu \alpha \alpha^{\prime}}(p, p+k) \Delta_{\alpha^{\prime} 3^{\prime}}(p+k) \Gamma_{1}^{\mu \beta^{\prime} 3}(p+k, p) \\
& =\frac{i e^{3}}{\left[(p+k)^{2}-M^{2}\right]}\left[(2 p+k)^{\nu} g^{\alpha \alpha^{\prime}}-p^{\alpha^{\prime}} g^{\nu \alpha}-(p+k)^{\alpha} g^{\nu \alpha^{\prime}}\right] \\
& {\left[g_{\alpha^{\prime} \beta^{\prime}}-\frac{(p+k)_{\alpha^{\prime}}(p+k)_{3^{\prime}}}{M^{2}}\right]\left[(2 p+k)^{\mu^{\prime}} g^{\beta^{\prime} \beta}-(p+k)^{\beta} g^{\mu \beta^{\prime}}-p^{\beta^{\prime}} g^{\mu \beta}\right]}
\end{aligned}
$$

temos, devido a contraçāo

$$
\begin{aligned}
& (p+k)_{\alpha^{\prime}}\left[(2 p+k)^{\nu} g^{\alpha \alpha^{\prime}}-p^{\alpha^{\prime}} g^{\nu \alpha}-(p+k)^{\alpha} g^{\nu \alpha^{\prime}}\right]=\left[p^{\nu} k^{\alpha}-g^{\nu \alpha} p . k\right] \\
& (p+k)_{\beta^{\prime}}\left[(2 p+k)^{\mu} g^{\beta^{\prime}, 3}-(p+k)^{\beta} g^{\mu \beta^{\prime}}-p^{\beta^{\prime}} g^{\mu, 3}\right]=\left[p^{\mu} k^{3}-g^{\mu \beta} p . k\right]
\end{aligned}
$$

que a potência em $k$ no termos que vem da primeira contribuição do propagador $\left(g_{\alpha^{\prime} 3^{\prime}}\right)$ é a mesma do termo $(p+k)_{\alpha^{\prime}}(p+k)_{B^{\prime}} / M T^{2}$. Assim o grau de divergência é quadrático, como definido na equação (4.44). Usando a regularização dimensional para a integração no momento interno, obtemos para a auto-energia do méson vetorial ${ }^{\bar{D}}$

$$
\Sigma^{\alpha 3}(p)=\frac{e^{2}}{8 \pi \theta} F(p) \varepsilon^{\alpha \beta \rho} p_{\rho},
$$

onde

$$
F(p)=\frac{\left(3 M^{2}+p^{2}\right)}{2 M p^{2}}\left[\left(M^{2}+p^{2}\right)-\frac{1}{2 M \sqrt{p^{2}}}\left(M^{2}-p^{2}\right)^{2} \log \left(\frac{|M|+\sqrt{p^{2}}}{|M|-\sqrt{p^{2}}}\right)\right] .
$$

Podemos ver que $\Sigma^{\alpha 3}$ nāo é zero para $p^{2}=M^{2}$, e que também não temos nenhum termo na Lagrangiana inicial que tenha a forma tensorial acima, de tal modo este termo possa ser absorvido por uma reparametrização ${ }^{6}$. Por outro lado, se introduzirmos um contra-termo com a forma tensorial dada pela equaçāo (4.47), de tal forma que propagador continue tendo pólo em $p^{2}=M^{2}$, obtemos

$$
\begin{aligned}
\Sigma_{R}^{\alpha 3}(p) & =\Sigma^{\alpha \beta}(p)-\delta M \varepsilon^{\alpha \beta \rho} p_{\rho} \\
& =\frac{e^{2}}{8 \pi \theta} F_{R}(p) \varepsilon^{\alpha \beta \rho} p_{\rho}
\end{aligned}
$$

\footnotetext{
${ }^{5}$ Veja apéndice $\mathrm{E}$.

${ }^{6}$ Vemos que $\Sigma(p)$ é divergente, no entanto com o uso da regularizaçāo dimensional essa divergência ficou obscurecida. Isso nos leva que por um processo de renormalizaçāo somos obrigados a incluir um termo de CS na Lagrangiana original. levando a presença de uma massa topológica.
} 
Com a imposição $\left.\Sigma_{R}^{\alpha \beta}(p)\right|_{p^{2}=M^{2}}=0$, temos

$$
\delta M=\frac{e^{2} M}{2 \pi \theta}
$$

e

$$
F(p)=\frac{\left(M^{2}-p^{2}\right)}{p^{2}}\left[\frac{\left(3 M^{2}-p^{2}\right)}{2 M}+\frac{\left(M^{2}-p^{2}\right)\left(3 M^{2}+p^{2}\right)}{4 M^{2} \sqrt{p^{2}}} \log \left(\frac{|M|-\sqrt{p^{2}}}{|M|+\sqrt{p^{2}}}\right)\right]
$$

O propagador renomalizado é dado por

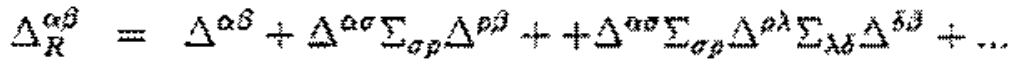

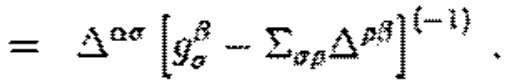

Usando as equaços para $\Sigma$ e para $A$, obtemos

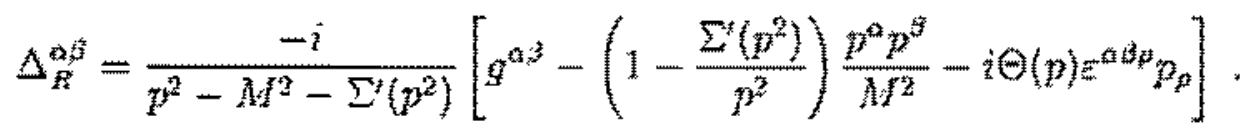

Na equaço acima temos que

$$
\Sigma^{\prime}\left(p^{2}\right)=\frac{e^{2}}{8 \pi \theta} \frac{p^{2} F_{n}^{2}(p)}{\left(p^{2}-M^{2}\right)} \quad \theta(p)=\frac{e^{2}}{8 \pi \theta} \frac{F_{r}(p)}{\left(p^{2}-M^{2}\right)}
$$

satisfazendo

$$
\left.\Sigma^{\prime}\left(p^{2}\right)\right|_{p^{2}=M^{2}}=0:\left.\quad F_{R}(p)\right|_{p^{2}=M^{2}}=0 ;\left.\quad \Theta(p)\right|_{p^{2}=M^{2}}=\frac{e^{2}}{8 \pi \theta M} .
$$

Observando o propagador renormalizado, equação (4.33), verificamos, devido a forma do áltimo termo, que temos a indução de um termo de Chern-mimons para o campo de natéria.

\subsubsection{Tensor de Polarizaçāo}

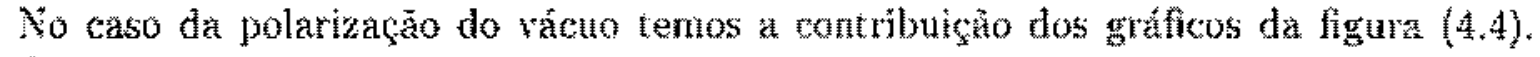
Araliticamente temos

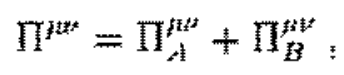

com

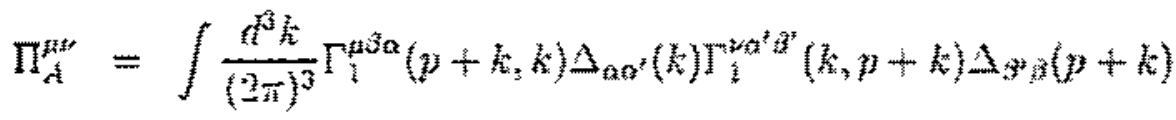

$$
\begin{aligned}
& \Pi_{B}^{t y}=\int \frac{d^{3} k}{(2 \pi)^{3}} \Gamma_{z}^{i t a b} \Delta_{a g}(k)
\end{aligned}
$$



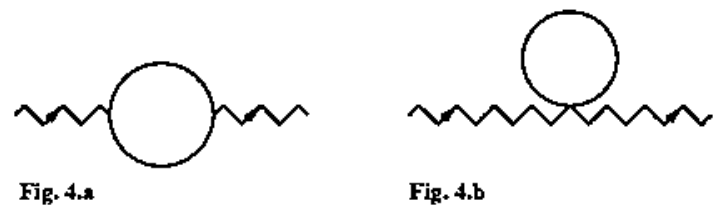

Figura 4.4: Polarização do Vácuo.

Novamente, usando a regularizaçāo dimensional, obtemos ${ }^{7}$

$$
\Pi^{\mu \nu}=\frac{i e^{2}}{8 \pi} \Pi\left(p^{2}\right)\left(g^{\mu \prime \prime}-\frac{p^{\mu} p^{\nu}}{p^{2}}\right)
$$

com $\Pi\left(p^{2}\right)$ dado por

$$
\Pi\left(p^{2}\right)=\frac{\left(p^{2}-4 M^{2}\right)}{4 M^{2}}\left[4|M|-\left(p^{2}+4 M^{2}\right) I_{0}\right],
$$

onde

$$
I_{0}=\int_{0}^{1} d x \frac{1}{\sqrt{M^{2}-p^{2} x(1-x)}}=\frac{1}{\sqrt{p^{2}}} \operatorname{Ln}\left[\frac{2 \sqrt{M^{2} p^{2}}+p^{2}}{2 \sqrt{M^{2} p^{2}}-p^{2}}\right] .
$$

Para $p^{2}<<M^{2}$, temos que $\Pi\left(p^{2}\right) \approx 4 p^{2} / 3 M$. Ou seja, temos a indução de um termo de Maxwell para o campo de $\mathrm{AB}$, como acontece nos casos de spin zero e 1/2.

\subsubsection{Correção de Vértice e Momento Magnético}

A correção de 1-loop para o vértice trilinear, é dada pelos très primeiros gráficos da figura (4.5). Os dois últimos gráficos serāo posteriormente usados no cálculo do momento magnético e da função de quatro pontos. Analiticamente o gráfico (4.5a) tem a forma

$$
\begin{aligned}
\Gamma_{a}^{\mu \alpha \beta}\left\langle p, p^{\prime}\right\rangle= & \int \frac{d^{3} k}{(2 \pi)^{3}}\left[\Gamma_{1}^{\rho \alpha \alpha^{\prime}}(p, p+k) \Delta_{\alpha^{\prime} \rho^{\prime}}(p+k)\right. \\
& \left.\Gamma_{1}^{\mu \rho^{\prime} \sigma^{\prime}}\left(p+k, p^{\prime}+k\right) \Delta_{\sigma^{\prime} \beta^{\prime}}\left(p^{\prime}+k\right) \Gamma_{1}^{\sigma \beta^{\prime} 3}\left(p^{\prime}+k, p^{\prime}\right) D_{\sigma \rho}(k)\right] .
\end{aligned}
$$

Usando as regras de Feynman temos

$$
\Gamma_{a}^{\mu \alpha \beta}\left(p, p^{\prime}\right)=\frac{i e^{3}}{(2 \pi)^{3} \theta} \int \frac{d^{3} k}{(2 \pi)^{3}} \frac{N_{0}^{\mu \alpha 3}}{\left[(p+k)^{2}-M^{2}\right]\left[\left(p^{\prime}+k\right)^{2}-M M^{2}\right]\left[k^{2}-\mu^{2}\right]},
$$

\footnotetext{
${ }^{7}$ veja apêndice $E$.
} 


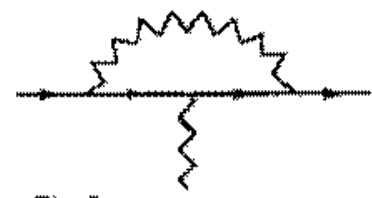

Fiv 5

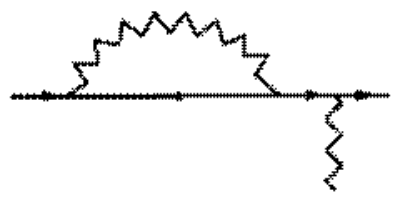

Hig 5.4

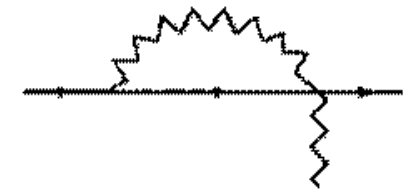

Fin

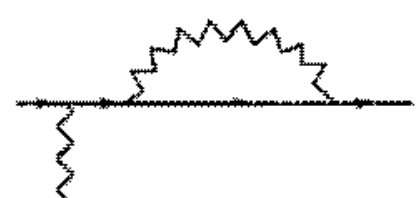

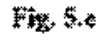

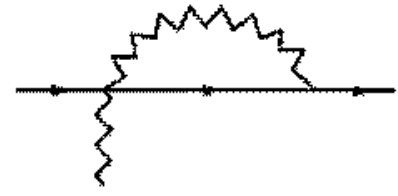

Fis

Figura 4.3̈: Correção le Vértice.

onde $f^{2}$ ê um corte infruvermelho que do tomado indo a zero no final dos cálculos, e

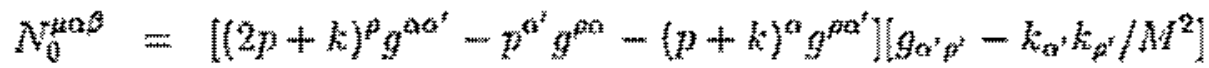

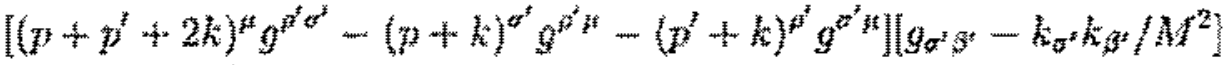

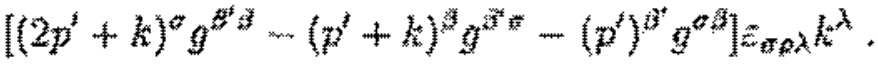

Com o auxilio da parametrizaçâo

$$
\frac{1}{a b c}=\int_{0}^{1} d x \int_{0}^{1} d y \frac{2 y}{(b-c) x y+(c-a) y+a]^{3}}
$$

podemos escrever (4.62) como

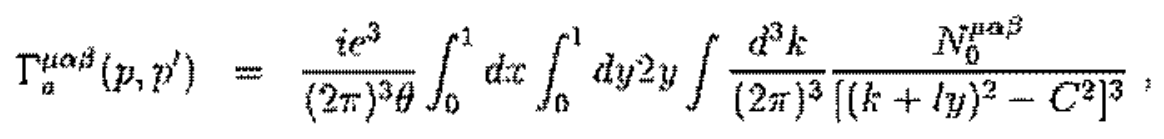

onde $l=p(1-x)+p x$ e $C^{2}=M^{2} y+\mu^{2}(1-y)-\left(y-p^{2}\right)^{2} x(1-x) y^{2}$. Fazendo a translação ${ }^{8}$ $k \rightarrow k-l y$ nós podemos reeserever $(4,65)$ como

$$
\Gamma_{a}^{\mu \alpha 3}\left(p, p^{\prime}\right)=\frac{i c^{3}}{(2 \pi)^{3}} \int_{0}^{1} d x \int_{0}^{1} d y 2 y \int \frac{d^{3} k}{(2 \pi)^{3}} \frac{N_{1}^{\mu \alpha a B}}{\left[k^{2}-C^{2}\right]^{3}}
$$

$\operatorname{com} N_{1}^{\mu \alpha 3}=\left.N_{0}^{\mu a 3}\right|_{k \rightarrow k-i y}$. Como o denominador é par em $k$ podemos eliminar os termos ímpares em $k$ no numerador. A integraçäo em $k$ é feita usando-se regularizaçäo dimensional[55]. As integraçöes paramétricas restantes são extremamente complicadas,

${ }^{8}$ Dexido ao grande múmero de termos que aparecem nesta parte do cálculo, nós usamos um programa de cálculo algébrico (Mathenatica). Os cálculos das funçöes de quatro pontos também sâo efetuadas con a ajuda do Sathematica de modo contrátio seriam intikeis. 
assim, para efetuá-las iremos para a concha de massa $\left(p^{2}=p^{2}=M^{2}\right)$ e faremos uma expansão para $Q=p-p^{t}$ pequeno, mantendo termos ate ordem $Q$. Nesta aproximação $C^{2}$ é independente de $x$, entäo a integraçăo nesta variável fica fácil de ser executada, Posteriormente podemos efetuar a integracaio em $y$. Neste ponto vemos que aparecem

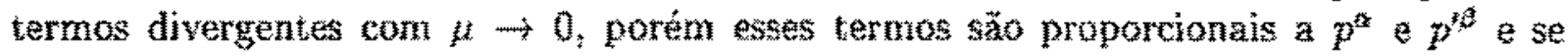
anulam quando contraidos com as polarizaçoes externas. Assim a menos destes termos obtemos até ordem $1 / M$,

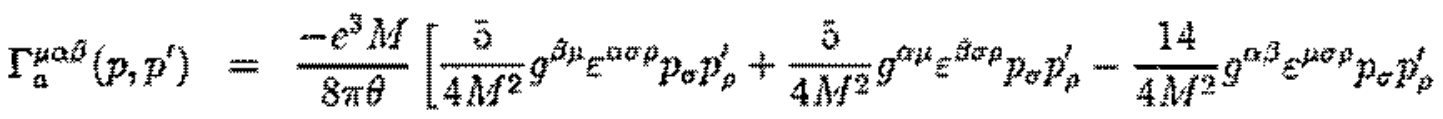

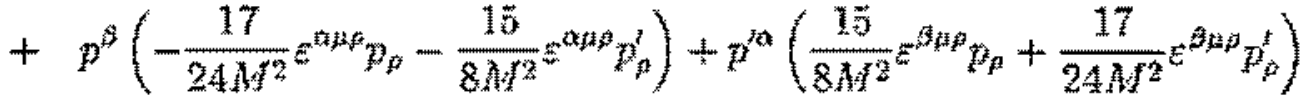

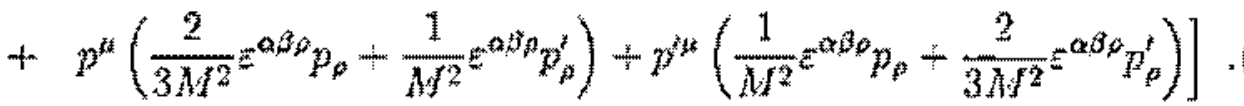

Os outros quatro gráficos da figura $(4.5)$ säo dados por

$$
\begin{aligned}
& \Gamma_{b}^{\mu \alpha \alpha^{3}}\left(p, p^{\prime}\right)=\int \frac{d^{3} k}{(2 \pi)^{3}}\left[\Gamma_{1}^{p \alpha \alpha^{2}}(p, p+k) \Delta_{\alpha^{\prime} S^{\prime}}(p+k) \Gamma_{2}^{\mu \alpha \theta^{2}{ }^{3}} D_{\sigma p}(k)\right] \\
& \Gamma_{c}^{\mu \alpha \beta}\left(p, p^{\prime}\right)=\int \frac{d^{3} k}{(2 \pi)^{3}}\left[\Gamma_{2}^{\mu \alpha \alpha \alpha^{\prime}} \Delta_{\alpha^{\prime}, p^{\prime}}\left(p^{\prime}+k\right) \Gamma_{1}^{\sigma \beta^{\prime} \beta}\left(p^{\prime}+k, p^{\prime}\right) D_{p \rho}(k)\right] \\
& \Gamma_{\alpha}^{\mu \alpha 3}\left(p, p^{\prime}\right)=\left[\Sigma_{R}^{\alpha \alpha^{\prime}}(p) \Delta_{a^{*}, z^{\prime}}(p) \Gamma_{\mid}^{\mu \beta^{\prime} \beta}\left(p, p^{\prime}\right)\right] \\
& \Gamma_{z}^{\mu \alpha, 3}\left(p, p^{\prime}\right)=\left[\Gamma_{1}^{\mu \alpha \alpha^{\prime}}\left(p, p^{\prime}\right) \Delta_{\alpha^{\prime} y^{\prime}}\left(p^{\prime}\right) \sum_{f}^{\beta^{\prime} \beta}\left(p^{\prime}\right)\right] .
\end{aligned}
$$

Os gráficos $(4,5 \mathrm{~b})$ e $(4.5 \mathrm{c})$ são feitos de mesmo modo que o gráfico (4.5a), porém os cálculos ficam mais simples pois a necessário apenas um parâmetro de Fevnman. Os gráficos (4.5d) e (4.5e) süo feitos com a ajuda da equacho (4.49). Na concha de massa e mantendo termos até orden $1 / M$, a menos de termos proporcionais a $p^{n}$ e $p^{73}$ que se. anulam quando contraidos pelas polarizaçoes, temos cque

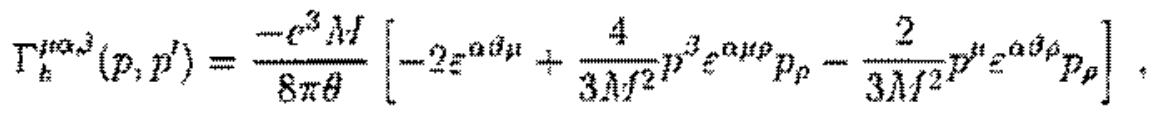

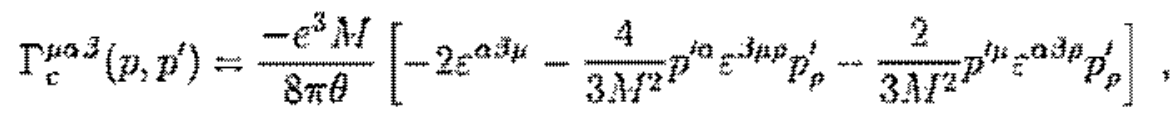




$$
\begin{gathered}
\Gamma_{d}^{\mu \alpha \beta}\left(p, p^{\prime}\right)=\frac{-e^{3} M}{8 \pi \theta}\left[-\frac{1}{M^{2}} p^{\beta} \varepsilon^{\alpha \mu \rho} p_{\rho}+\frac{1}{M^{2}} g^{\mu \beta} \varepsilon^{\alpha \sigma \rho} p_{\sigma} p_{\rho}^{\prime}+\frac{\left(p+p^{\prime}\right)^{\mu}}{M^{2}} \varepsilon^{\alpha \beta \rho} p_{\rho}\right], \\
\Gamma_{e}^{\mu \alpha \beta}\left(p, p^{\prime}\right)=\frac{-e^{3} M I}{8 \pi \theta}\left[\frac{1}{M^{2}} p^{\prime \alpha} \varepsilon^{\beta \mu \rho} p_{\rho}^{\prime}+\frac{1}{M^{2}} g^{\mu \alpha} \varepsilon^{\beta \sigma \rho} p_{\sigma} p_{\rho}^{\prime}+\frac{\left(p+p^{\prime}\right)^{\mu}}{M^{2}} \varepsilon^{\alpha \beta \rho} p_{\rho}^{\prime}\right] .
\end{gathered}
$$

A correção do vértice quadrilinear não contribuirá para a amplitude de espalhamento em 1-loop e nem para o momento magnético. Além disso, como visto pela equação (4.44), as divergências destes gráficos são logaritímicas. Porém o espaço de fase é ímpar, então essas divergências se cancelam pela simetria da integração, tornando esses gráficos finitos. É fácil de verificar de (4.47), (4.67), (4.72) e (4.73) que a identidade de Ward é satisfeita, ou seja,

$$
\left.e \frac{d \Sigma^{\alpha \beta}(p-q)}{d(q-p)^{\mu}}\right|_{q=0, p^{2}=M^{2}}=\Gamma_{a}^{\mu \alpha \beta}(p, p)+\Gamma_{b}^{\mu \alpha, 3}(p: p)+\Gamma_{c}^{\mu \alpha \beta}(p, p) .
$$

Podemos também calcular a correçāo para o momento magnético do méson vetorial. Isso é feito acoplando um campo externo ao campo de matéria. Porém para esse cálculo é conveniente irmos para o referencial de Breit[53, 59, 60]. Neste referencial o momento da partícula final é na mesma direção da partícula inicial, porém no sentido contrário $\left(\mathbf{p}=-\mathbf{p}^{\prime}\right)$. O momento magnético para spin $s= \pm 1$ está relacionado con a funçāo de vértice por $[60]$

$$
\mu^{i}=\operatorname{Lim}_{q \rightarrow 0} \frac{1}{|q|} \frac{1}{2 w_{p}} \Gamma^{i}(\mathbf{p},-\mathbf{p})
$$

onde

$$
\Gamma^{\prime}(\mathrm{p} .-\mathrm{p})=\varepsilon_{3}^{*}(-\mathbf{p}, s) \Gamma_{1}^{i \alpha^{3}}(\mathbf{p},-\mathbf{p}) \varepsilon_{\mathrm{\alpha}}(\mathbf{p}, s)
$$

e $q=p-p^{\prime}=2 \mathrm{p}$ é o momento transferido. A prescrição do limite $q \rightarrow 0$ serve para reter somente o termo proporcional a $q$. A nivel de árvore e em ordem dominante em $\mathrm{p} / M$, obtemos de $(4.22),(4.78)$ e usando (4.27) que

$$
\Gamma^{i}(\mathrm{p},-\mathrm{p})=\operatorname{se} \varepsilon^{i j} q_{j}
$$

isto é, o momento magnético para spin $s= \pm l$ é 


$$
\mu=\frac{e}{2 M} g s= \pm \frac{e}{2 M} .
$$

lavando a un fator giromagnético $g=1$. Para obtemos um fator $g=2$ terímos que adicionar em (4.18) o termo de momento magnetico[50]

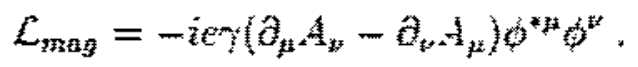

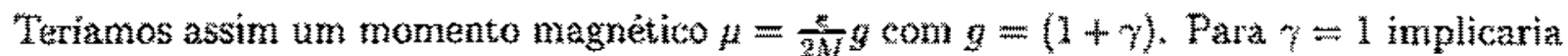

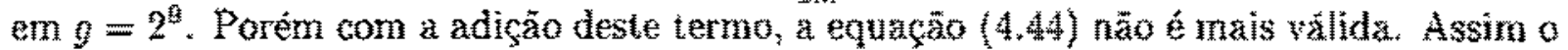
comportamento ruim do propagador no ultravioleta leva a termos com altas diveryência

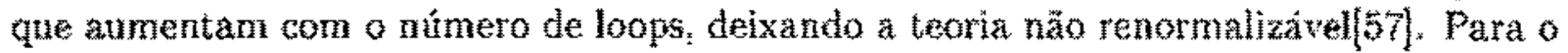
caso de $y=2$ talvez seja comveniente tratar o problena com uma teoria nab-Abeliana[21]. Poróm isso implicaria en tma análise datalhada de todas az conseguéncia resultantes da teoria năo-dbeljana. Devido a estas consideraçòes nós continuaremos nossa analise sem incluirmos o terno (4.81) na lagrangiana original.

A correça para o momento magnético a 1-loop é dada pelos grafocos da figura (4.5). Usando as equaçós $(4.67) \mathrm{e}(4.72) \cdot(4.75)$ temos

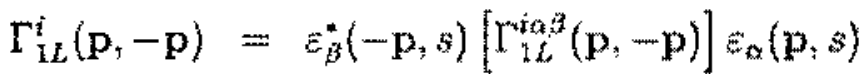

$$
\begin{aligned}
& \Gamma_{L}^{i}(\mathbf{p},-\mathbf{p})=\frac{\varepsilon^{3}}{8 \pi \theta} \varepsilon^{i j} q_{j}
\end{aligned}
$$

Isso nos dá para o momento magnético até 1-loop

$$
\mu= \pm \frac{e}{2 M}\left[1 \pm \frac{e^{2}}{8 \pi \theta}\right]
$$

Observe qué a corregåo é a mesma independentemente do spin. Portanto, como no caso dé spin 1/2 [25], ela gena um actéscimo no momento magnético positivo e um dectéscimo no momento niagnético negativo.

\subsubsection{Espalhamento}

Para a correcão da amplitude de espalhamento an 1-loop e netessario o cálulo dos graficos da figma (4.6). Os gráfcos de (4.6.1) a (4.6.6) anesar de muito trabalhosos sảo possiveis de serem calculados. Porém os gráficos (4.6.7) e (4.6.8) mesmo na concha de

\footnotetext{
Gvidencias experimentais e cálculos a nivel de árores de um particula interatgindo com um campo externo en $(3+1) \mathrm{D}$ prevér $g \approx 2[58]$
} 
massa e na aproximação $\mathrm{p} / \mathrm{M}$ se tornam inviáveis. Isto se deve ao lato que as integraçōes parametricas resultantes, quando aplicada a parametrizaçăo de Feynman, se tornam muito complicadas ${ }^{10}$.

A contribuiçăo dominante que vem da correça de vértice estäo representadas nos gráficos (4.6.1) (4.6.2). Usando as equaçóes $(4.67), 0(4.72)$ a $(4.75)$ obtemos para o graffico $(4.6 .1)$

$$
\begin{aligned}
& M_{1}^{s_{1}=1, s_{2}=1, z_{3}, v_{4}}=\frac{i \epsilon^{4} M}{8 \pi \theta^{2}}(\cos \alpha-i \sin \alpha)\left\{\frac { 1 + s _ { 4 } } { 1 - \operatorname { c o s } \alpha } \left[-2+\left(1-s_{3}\right) \cos \alpha\right.\right. \\
& \left.\left.+\left(1+s_{3}\right) \cos ^{2} \alpha-i\left(1+s_{3}\right) \sin \alpha \cos \alpha\right]\right\} \\
& \mathcal{M}_{1}^{s_{1}=-1, s_{2}=-1, s_{3}, s_{4}}=\frac{i e^{4} M}{8 \pi \theta^{2}}(\cos \alpha+i \sin \alpha)\left\{\frac { - 1 + s _ { 4 } } { 1 - \operatorname { c o s } \alpha } \left[2-\left(1+s_{3}\right) \cos \alpha\right.\right. \\
& \left.\left.-\left(1-s_{3}\right) \cos ^{2} \alpha-i\left(1-s_{3}\right) \sin \alpha \cos \alpha\right]\right\} \\
& \mathcal{M}_{1}^{s_{1}=1, s_{2}=-1, s_{3}, 8_{4}}=\frac{i c^{4} M}{8 \pi \theta^{2}}\left\{\frac{-1+s_{4}}{1-\cos \alpha}\left[(]+s_{3}\right\}-2 s_{3} \cos \alpha\right. \\
& \left.-\left(1-s_{3}\right) \cos ^{2} \alpha+2 i \sin \alpha-\left(1-s_{3}\right) \sin \alpha \cos \alpha\right\}
\end{aligned}
$$

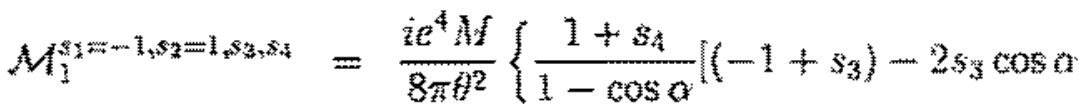

$$
\begin{aligned}
& \left.\left.+\left(1+s_{3}\right) \cos ^{2} \alpha+2 i \sin \alpha-\left(1+s_{3}\right) \sin \alpha \cos \alpha\right)\right\} \text {. }
\end{aligned}
$$

O gráfico (4.6.2) nos leva a

$$
\begin{aligned}
\mathcal{M}_{2}^{s_{2}=i_{1}, z_{3}=1, s_{3}, s_{4}}= & \frac{i e^{4} M}{8 \pi \theta^{2}}(\cos \alpha-i \sin \alpha)\left\{\frac { 1 + s _ { 3 } } { 1 - \operatorname { c o s } \alpha } \left[-2+\left(1-s_{4}\right) \cos \alpha\right.\right. \\
& \left.\left.+\left(1+s_{4}\right) \cos ^{2} \alpha-i\left(1+s_{4}\right) \sin \alpha \cos \alpha\right]\right\} \\
\mathcal{M}_{2}^{s_{1}=-1, s_{2}=-1, s_{3}, s_{4}}= & \frac{i e^{4} M}{8 \pi \theta^{2}}(\cos \alpha+i \sin \alpha)\left\{\frac { - 1 + s _ { 3 } } { 1 - \operatorname { c o s } \alpha } \left[2-\left(1+s_{4}\right) \cos \alpha\right.\right. \\
& \left.\left.-\left(1-s_{4}\right) \cos ^{2} \alpha-i\left(1-s_{4}\right) \sin \alpha \cos \alpha\right]\right\}
\end{aligned}
$$

\footnotetext{
10 No apêndice E nós explicitaremos o cálculo de alguns gráfos
} 


$$
\begin{aligned}
& M_{2}^{s_{1}=1_{4} s_{2}=-w_{3}, s_{3} s_{4}}=\frac{i e^{4} M}{8 \pi \theta^{2}}\left\{\frac { 1 + s _ { 3 } } { 1 - \operatorname { c o s } \alpha } \left[\left(-1+s_{4}\right)-2 s_{4} \cos \alpha\right.\right. \\
& \left.\left.+\left(1+s_{1}\right) \cos ^{2} \alpha+2 i \sin \alpha-i\left(1+s_{4}\right) \sin \alpha \cos \alpha\right]\right\} \\
& M_{2}^{s_{1}=-1_{1} s_{2}=1, u_{3}, s_{4}}=\frac{i e^{A} A}{8 \pi \theta^{2}}\left\{\frac { - 1 + s _ { 3 } } { 1 - \operatorname { c o s } \alpha } \left(\left\{1+s_{4}\right)-2 s_{4} \cos \alpha\right.\right. \\
& \left.\left.-\left(1-s_{4}\right) \cos ^{2} \alpha+2 i \sin \alpha-i\left(1-s_{4}\right) \sin \alpha \cos \alpha\right]\right\}
\end{aligned}
$$

A contribuiçăo dominante que vem da polarizaçăo do văcuo está representada no gráfico (4.6.3). Usando a equatüo $(4.58)$ temos

$$
\begin{aligned}
& M_{3}^{x_{1}, s_{2}, s_{3}, s_{4}}=\frac{i e^{4} M}{8 \pi g^{2}}\left\{\frac{16}{3}\left[\left(1+s_{1} s_{3}\right) \cos \alpha-i\left(s_{1}+s_{3}\right) \sin a\right\}\right. \\
& \left.\left[\left(1+s_{2} z_{4}\right) \cos \alpha-i\left(s_{2}+3_{4}\right) \sin \alpha\right]\right\} \text {. }
\end{aligned}
$$

O gráfeo $(4.6 .4)$ édado por

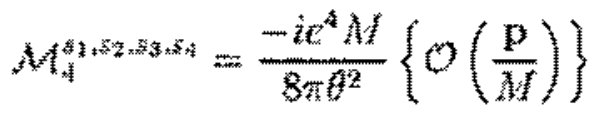

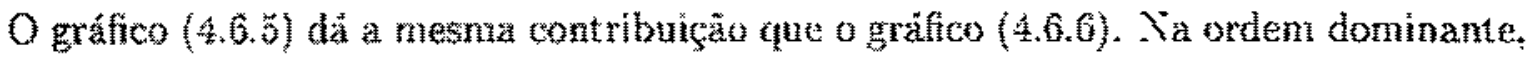

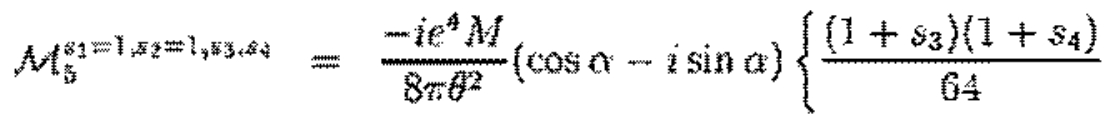

$$
\begin{aligned}
& [-2 x+55 \ln (3)](\cos \alpha-i \sin \alpha)\}
\end{aligned}
$$

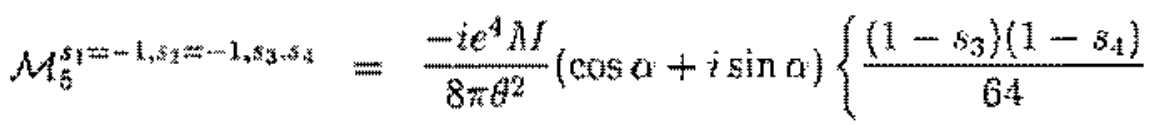

$$
\begin{aligned}
& \{-28+53 \ln (3))(\cos \alpha+i \sin \alpha)\} \\
& \mathcal{M}_{5}^{s_{2}=1_{N_{2}}=w_{2}=s_{3} s_{4}}=\frac{-i e^{4} M}{8 \pi \theta^{2}}\left\{\left(-1+s_{3} s_{4}\right)[-36+73 \ln (3)]\right. \\
& \left.+\left(s_{3}-s_{4}\right)\{-20+37 \ln (3)]\right\}
\end{aligned}
$$




$$
\begin{aligned}
\mathcal{M}_{5}^{s_{1}=-1, s_{2}=i, s_{3}, s_{4}}= & \frac{-i e^{4} M}{8 \pi \theta^{2}}\left\{\left(1-s_{3} s_{4}\right) \mid-36+73 \ln (3)\right] \\
& \left.-\left(s_{3}-s_{4}\right)[-20+37 \ln (3)]\right\}
\end{aligned}
$$

Para os grăficos (4.6.7) e (4.6.8), apesar de nâa chegarmos a um tesultado final, podemos verficar que 0 termo de mais aita poténcia na varável de integraçäo é dado por

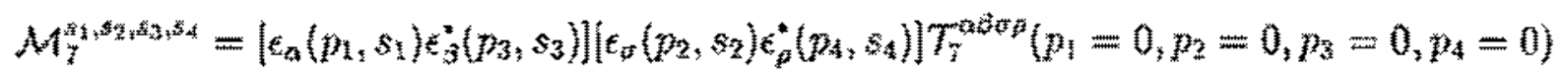

onde

$$
T_{7}^{o d p_{p}}\left(p_{1}=0, p_{2}=0, p_{3}=0, p_{4}=0\right)=\frac{e^{4}}{\theta^{2}} \int \frac{t^{3} k}{(2 \pi)^{3} k^{2}\left[k^{2}-A^{2}\right]^{2}}
$$

Igualmente para o gráfico (4.15.8)

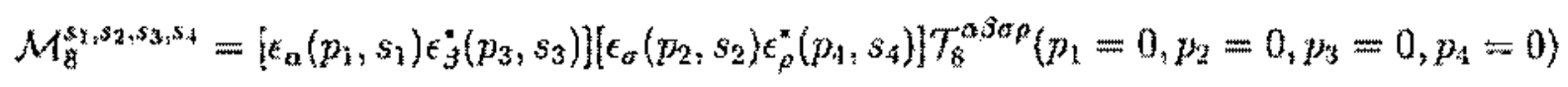

onde

$$
\mathcal{T}_{\mathrm{g}}^{\alpha \sigma_{\sigma o}}\left(p_{1}=0, p_{2}=0, p_{3}=0, p_{4}=0\right)=\frac{e^{4}}{\theta^{2}} \int \frac{d^{3} k}{(2 \pi)^{3}} \frac{\left[2 k^{a} k^{3} k^{\alpha} k^{p}\right]}{k^{2}\left[k^{2}-M M^{2}\right]^{2}}
$$

Vemos assim que a divergència destes dois gráficos élinear, do mesmo modo dos gráficos anteriores, que devido a regularizaçāo dimensional tornam-se finitos.

\subsection{Análise}

Nós observamos que os modelos de Proca e MCS quando acoplades tom CS, levam a resultados levemente diferentes para amplitude de espalhamento a nivel de arvort. Se a analise for estendida a I-loop, começam a apareser problemas devido to comportamento ultravioleta, que difere nos dois casos. Especificamente, no espalhamenio en 1-loop, os cálculos começan a ser impraticáveis, principalmente no modelo de MCS. Neste caso o número de parâmetros de Feynman toma as integraçóes parametricas muito difíceis de serem efetuadas. Para o modelo de Proca, o cálculo em 1-loop, relativo as parametrizaçōes, fica um pouco mais fảcil. Porém, devido a forma das regras de Feynman, os integrandos 

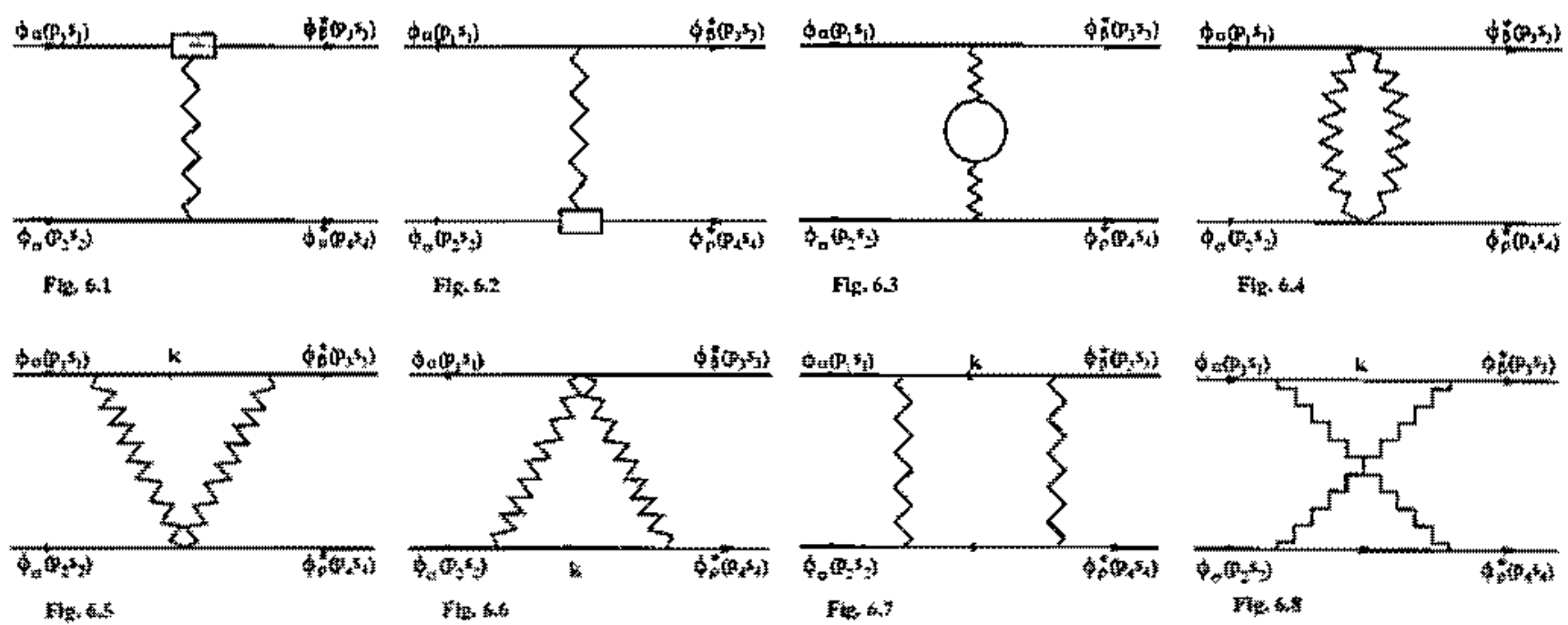

Figurat 46: Espalhamento 1-ioop.

ficam extensos, fazendo com que o desenvolvimento da álgebra, só se torne possixel com a ajuda de um programa de cálculo algébrico. Mesmo năo tendo calculado explieitamente todas as contribuiçôes para a amplitude de espalhanento em l-loop, conseguimos fazer uma análise dos gráficos que apresentam divergências. Obtemos assin, além da correçăo para a auto-energia e polarizaçäo do vácuo, a correçăo para o momento nagnético. Como no caso de spin $1 / 2$, esta correção é a mesma independentemente do sinal do spin. Para função de quatro pontos do campo de matéria: calculamos explicitamente os gráficos que poderiam apresentar divergências. Para os gráficos box e cross-box (figura $(4.6 .7-8)$ ) cálculo completo näo pode ser concluído pela forma da integrais paramétricas finais e devido a extensầo do integrando. 


\section{Conclusão}

Nesta tese abordamos algumas teorias contendo o termo de Chern-Simons e outros campos para vários valores de spin. Procuramos identificar relaçōes entre modelos e também fizemos una análise do limite nāo relativistico nos processos de espalhamento de duas partículas.

No primeiro capítulo estudamos o problema da equivalência de modelos de gauge quando acoplados a outro campo dinâmico, neste caso o campo de Dirac. Como vimos, para que a equivalência entre os modelos Self-Dual e Maxwell-Chern-Simons permaneça válida quando interagindo com férmions, é necessário termos acoplamentos específicos e diferentes em cada modelo.

Nos capítulos 2 e 3 verificamos explicitamente qual a influência de adicionarmos um índice de simetria interna para o campo de matéria acoplado com Chern-Simons, isto é, nós estudamos o efeito Aharonor-Bohm não-Abeliano para partículas de spin 0 e $1 / 2$. $O$ cálculo foi feito no gauge de Coulomb e usando o procedimento de corte intermediário, com o objetivo de facilitar a comparação com o resultado da teoria não relativística e também com o caso Abeliano.

No caso de spin 0 , verificamos que o setor de alta energia funciona como contra-termo natural para o setor de baixa energia, deixando a teoria finita. No caso da teoria nāo relativística escalar é necessário a introduçāo de um corte, que só é eliminado para um valor crítico da constante de acoplamento do termo de auto-interação. Temos também que a teoria relativística, além de reproduzir o resultado nào relativístico em ordem dominante, dá em ordem sub-dominante contribuiçòes extras que vem das correções radiativas. Estas contribuições extras poderiam em princípio ser observadas por experimentos mais refinados. Esses termos também poderiam ser adicionados na Lagrangeana da teoria não relativísticas no mesmo espírito de teorias efetivas.

No caso de spin $1 / 2$, nós nos detemos na análise da contribuiçăo dominante. No caso da teoria nāo relativística vemos que os termos dependentes do corte $\lambda_{N R}$ se cancelam. Isto devido ao fato que para os férmions temos o termo de interação de Pauli que faz o mesmo papel do termo de interação de contato da teoria escalar. No caso relativístico, nós também obtemos as correçōes radiativas para a auto-energia, tensor de polarização e para o vértice trilinear. No cálculo do vértice obtemos a correção para momento magnético do férmion. Vimos que ele difere do caso Abeliano. devido ao fator de grupo, no entanto 
no limite Abeliano $(g=e \sqrt{2}$ e $T=i / \sqrt{2})$ recuperamos o resultado da referência [23]. No caso do espalhamento, o resultado relativístico nos dá contribuições extras que não aparecem no caso nào relativístico.

Além disso, observamos que aparecem gráficos extras na teoria nảo-Abeliana, tanto escalar quanto espinorial, cujo papel básico é cancelar as contribuições adicionais, que vem devido aos campos fantasmas e também se somarem as contribuições que surgem devido a estrutura de grupo nos gráficos que igualmente estão presentes na teoria Abeliana.

No último capítulo nós exploramos o fato de que em três dimensões o campo de gauge massivo e carregado de spin 1, pode ser descrito por várias formulações, que no caso livre estão aparentemente relacionadas. Quando esses modelos são acoplados com um potencial tipo Aharonov-Bohm verificamos que, mesmo na aproximaçāo de árvore, eles apresentam algumas diferenças, como visto pelos resultados da equaçōes (4.32) e (4.41). Vimos também, que na amplitude de espalhamento a nivel de árvore, temos um termo que pode ser interpretado como uma interaçāo magnética, de tal forma que quando espalhamos partículas de spins anti-paralelos este termo se cancela, produzindo o mesmo resultado que o espalhamento de partículas sem spin. Nesse capítulo também implementamos a análise dos gráficos potencialmente divergentes, até 1-loop, para o modelo de Proca minimamente acoplado com Chern-Simons. onde obtivemos além da auto-energia e polarização do vácuo a correção ao momento magnético. 


\section{Apêndice A}

\section{Tensor de Polarização para $M C S$ com Acoplamento Magnético}

Para o cálculo do tensor de polarização na expansāo $1 / N$, para o modelo de $M C S$ com acoplamento magnético, nós redefinimos a constante de acoplamento $e->\alpha / \sqrt{N}$, tal que a o termo de interação fica,

$$
\mathcal{L}_{I}=\frac{\alpha}{m \sqrt{N}} \varepsilon_{\mu \alpha \beta} \partial^{\alpha} A^{3} \bar{\psi}_{a} \gamma^{\mu} \psi_{n} .
$$

Assim o tensor de polarização é dado por

$$
\Pi_{M C S}^{\mu \nu}=-t r \int \frac{d^{3} q}{\left(2 \pi^{3}\right)}\left[\left(\frac{-i \alpha}{m \sqrt{N}}\right)^{2} \gamma_{\beta} \varepsilon^{B \alpha \mu}\left(i k_{\alpha}\right) S(k+q) \gamma_{\sigma} \varepsilon^{\sigma \rho \nu}\left(-i k_{\rho}\right) S(q)\right] .
$$

Como vemos aqui, a contribuição que vem devido aos vértices, nāo depende do momento de integraçāo, o que leva ao grau de divergência da integral acima a $d(\gamma)=1$ como dado pela equaçāo (1.45). Usando a forma dos propagadores e após efetuarmos o traço podemos escrever

$$
\begin{aligned}
\Pi_{M C S}^{\mu \nu}= & N\left(\frac{-i \alpha}{m \sqrt{N}}\right)^{2} \int \frac{d^{3} q}{\left(2 \pi^{3}\right)}\left\{\frac { \varepsilon ^ { 3 \alpha \mu } \Xi ^ { \sigma \rho \nu } } { [ ( k + q ) ^ { 2 } - M ^ { 2 } ] [ q ^ { 2 } - M ^ { 2 } ] } \left[2 k_{\alpha} k_{\rho} q_{B} q_{\sigma}\right.\right. \\
& \left.\left.-k_{\alpha} k_{\rho}\left(k \cdot q+q^{2}\right) g_{B \sigma}-i N \varepsilon_{3 \sigma \lambda} k_{\alpha} k_{\rho} k^{\lambda}+M^{2} k_{\alpha} k_{\rho} g_{B \sigma}\right]\right\} .
\end{aligned}
$$

O fator $N$ multiplicando vem da efetuação do traço. Lsando agora o truque de Feynman

$$
\frac{1}{A B}=\int_{0}^{1} d x \frac{1}{[(A-B) x+B]^{2}}
$$


obtemos, após usar a regularização dimensional para a integração no momento interno,

$$
\Pi_{M C S}^{u k}=\frac{-i \alpha^{2}}{4 \pi} \int_{0}^{1} d x \frac{k^{2}}{m^{2}}\left[\frac{P^{\mu u} 2 k^{2} x(1-x)-i M \varepsilon^{\mu \nu \rho k_{\rho}}}{\left[M^{2}-k^{2} x(1-x)\right]^{1 / 2}}\right]
$$

onde $P^{\mu \nu}=g^{\alpha \nu}-k^{\mu} k^{\nu} / k^{2}$. Podenos ainda escrever $\Pi^{\mu \nu}$ de uma forma mais simples,

$$
\Pi_{M C S}^{\mu \nu}=-i 0^{2} \frac{k^{2}}{m^{2}}\left[\Pi_{R}\left(k^{2}\right) P^{\mu \prime \prime}-i M \mathrm{I}_{0} E^{\mu \nu \rho \rho} k_{\rho}\right],
$$

conl

$$
\begin{aligned}
& \Pi_{e}=\frac{1}{4 \pi} \int_{0}^{1} d x \frac{2 k^{2} x(1-x)}{\left.M^{2}-k^{2} x(1-x)\right]^{1 / 2}}, \\
& \Pi_{0}=\frac{1}{4 \pi} \int_{0}^{1} d x \frac{1}{\left.M^{2}-k^{2} x(1-x)\right]^{1 / 2}} .
\end{aligned}
$$




\section{Apêndice B}

\section{Integrais}

Neste apêndice vamos escrever algumas relaçöes e integrais usadas no cálculo dos gráficos do capítulo 3 e 4 . Xos gráficos de 1-Loop, apés a integracăo en $k^{\natural}$, ficamos com integrais do tipo

$$
\int_{0}^{\infty} d(\mathbf{k})^{2} \int_{0}^{2 \pi} d \alpha f\left(\mathbf{p}, \mathbf{p}^{\prime}, \mathbf{k}, m\right)
$$

De acordo com a figura (B.1) temos que

$$
\begin{aligned}
\mathbf{p} \cdot \mathbf{p}^{\prime} & =|\mathbf{p}|^{2} \cos \theta \\
\mathbf{p} \wedge \mathbf{p}^{\prime} & =|\mathbf{p}|^{2} \sin \theta \\
\mathbf{p} \cdot \mathbf{k} & =|\mathbf{p}||\mathbf{k}| \cos \{\alpha+\theta / 2) \\
\mathbf{p}^{\prime} \cdot \mathbf{k} & =|\mathbf{p}||\mathbf{k}| \cos (\alpha-\theta / 2) \\
\mathbf{p} \wedge \mathbf{k} & =|\mathbf{p}||\mathbf{k}| \sin (\alpha+\theta / 2) \\
\mathbf{p}^{\prime} \wedge \mathbf{k} & =|\mathbf{p}||\mathbf{k}| \sin (\alpha-\theta / 2)
\end{aligned}
$$

Para a integração angular ( $\alpha$ ), usando as relaçöes acima teremos integrais do tipo[67].

$$
I_{n}=\int_{0}^{2 \pi} d a \frac{\cos (n \alpha)}{[2 \cos (0-\theta / 2)-f](2 \cos (\alpha+\theta / 2)-f]} .
$$

$\operatorname{com} n=0,1$ e 2, sendo $f=\left(\mathrm{k}^{2}+\mathrm{p}^{2}\right) /\|\mathrm{k}\| \mathrm{p} \|$. Fazendo a substituiç̆o $z=\exp (\mathrm{i} \omega)$ e $t=\exp (i 0 / 2)$ e usando o teorena dos residuos obtemos [28, 67]

$$
I_{n}=\frac{2 \pi}{B \sqrt{I^{2}-4}} \frac{1}{\sin (0 / 2)}\left[Z^{n-1} \sin [(n+1) \theta / 2]-Z^{n+1} \sin [(n-1) \theta / 2]\right]
$$




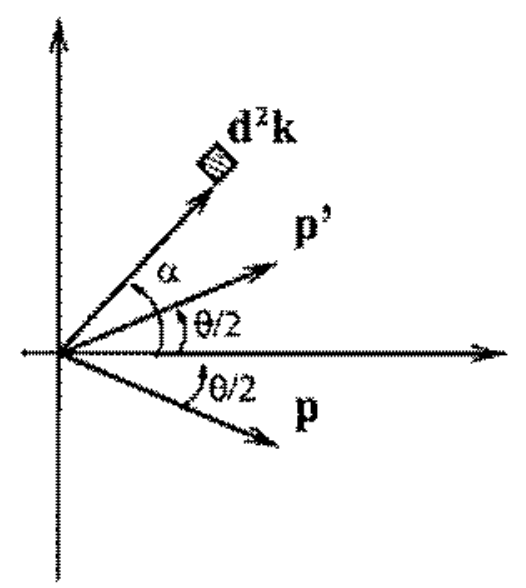

Figura B.I: $\mathrm{p}_{1}=\mathrm{p}_{1} \mathrm{p}_{3}=\mathrm{p}^{\prime}$ e $\theta$ é o änqulo de espalhamento.

sendo $B=f^{2}-2(1+\cos \theta)$ e $Z=\frac{1}{2}\left[f-\sqrt{f^{2}-4}\right]$. Assim

$$
\begin{aligned}
& I_{0}=\frac{2 \pi f}{B \sqrt{\int^{2}-4}}=\frac{2 \pi\left(\mathbf{k}^{2}+\mathrm{p}^{2}\right) \mathbf{k}^{2} \mathrm{p}^{2}}{\left|\mathrm{k}^{2}-\mathrm{p}^{2}\right|\left(\mathbf{k}^{3}+\mathbf{p}^{1}-2 \mathbf{p}^{2} \mathrm{k}^{2} \cos \theta\right)} \\
& I_{1}=\frac{2 \cos \theta / 2}{f} I_{0} \\
& I_{2}=I_{0}-\frac{\pi f}{\sqrt{f^{2} \ldots+4}}+\pi=\left(1-\frac{B}{2}\right) I_{0}+\pi .
\end{aligned}
$$

Após a integraçäo angular, e separando na região de baixa e alta energia, nos resta as integrais do tipo,

$$
\int_{0}^{\Lambda^{2}} d(\mathbf{k})^{2} f\left(|\mathbf{p}|, \mathbf{k}^{2}, m\right)+\int_{\Lambda^{2}}^{A_{0}^{2}} d(\mathbf{k})^{2} f\left(|\mathbf{p}|, \mathbf{k}^{2}, m\right)
$$

com $\Lambda_{0}$ un cutoff ultravioleta. No setor de baixa energia, faremos uma expansão em $1 / \mathrm{m}$, assirn nos resta integrais do tipo[60] (para $D=x^{2}+b x+a$ e $\Delta=4 a-b^{2}$ )

$$
\begin{aligned}
& I_{0}=\int d x \frac{1}{D}=\frac{2}{\sqrt{\Delta}} \operatorname{arctg}\left(\frac{b+2 x}{\sqrt{\Delta}}\right) \quad \Delta>0 \\
& I_{1}=\int d x \frac{x}{D}=\frac{1}{2} \log D-\frac{b}{2} I_{10} \\
& I_{2}=\int d x \frac{x^{2}}{D}=x-b I_{1}-a I_{0} .
\end{aligned}
$$

Para $x=\mathrm{k}^{2}, a=\mathrm{p}^{4} \mathrm{e} b=2 \mathrm{p}^{2} \cos \theta$ temos $\left(\mathrm{p}^{2}<<\Lambda^{2}\right)$ ate ordem $\eta^{2}\left(\eta \approx \frac{\mathrm{p}}{m} \approx \frac{\Lambda^{2}}{m^{2}} \approx \frac{\mathrm{p}^{2}}{\hbar^{2}}\right)$,

$$
I_{0}\left(p^{2}, A^{2}\right)-I_{0}\left(0, p^{2}\right)=-\frac{1}{p^{2}}\left[\frac{p^{2}}{A^{2}}+\frac{p^{2}}{A^{2}} \cos \theta+\ldots\right]
$$




$$
I_{1}\left(p^{2}, A^{2}\right)-I_{1}\left(0, p^{2}\right)=\log \frac{A^{2}}{q^{2}}-2 \cos \theta \frac{p^{2}}{\Lambda^{2}}+\left(1-4 \cos ^{2} \theta\right) \frac{p^{4}}{2 A^{4}}+\ldots
$$

No setor de alta energia faremos expansóes em torno de $p \approx 0$, e obteremos integrais do tipol69)

$$
\begin{aligned}
& \mathcal{J}_{1}=\int \frac{d x}{\sqrt{x+m^{2}}}=2 \sqrt{x+m^{2}} \\
& \mathcal{J}_{1}=\int \frac{d x}{x \sqrt{x+m^{2}}}=\frac{1}{m} \log \left(\frac{\sqrt{m^{2}+x}-\sqrt{m^{2}}}{\sqrt{m^{2}+x}+\sqrt{m^{2}}}\right) \\
& \mathcal{J}_{2}=\int \frac{d x}{x^{2} \sqrt{x+m^{2}}}=-\frac{\sqrt{m^{2}+x}}{m^{2} x}-\frac{1}{2 m^{2}} \mathcal{J}_{1} .
\end{aligned}
$$

Com $x=\mathbf{k}^{2}$ obtemos das equaçòes acima que (para $A<<m$ ) até ordem $\eta^{2}$,

$$
I_{1}\left(\Lambda^{2}, \Lambda_{0}^{2}\right)=\frac{1}{m}\left[-\log \left(\frac{\Lambda^{2}}{4 m^{2}}\right)+\frac{\Lambda^{2}}{2 m^{2}}-\frac{3 \Lambda^{4}}{16 m m^{4}}+\ldots\right]
$$




\section{Apêndice $\mathrm{C}$}

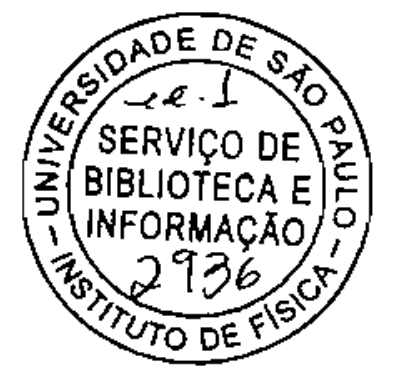

\section{Espalhamento em 1-Loop (Spin 0)}

Neste apêndice vamos explicitar o cálculo em 1-loop do espalhamento Aharonov-Bohm não-Abeliano para partículas escalares. Detalharemos o caso gráfico (2.3b) no caso nāo relativitico, e o gráfico (2.11.c4) no caso relativístico.

- Gráfico (2.3.b)

O gráfico (2.3b) é dado por

$$
\mathcal{M}_{b}(\theta)=-i\left(\frac{2 g^{2}}{m \Theta}\right)^{2}\left[T^{c} T^{a} \otimes T_{c} T_{a}\right] \int \frac{d^{2} \mathbf{k}}{(2 \pi)^{2}} \frac{\left(\mathbf{p}_{1} \wedge \mathbf{k}\right)\left(\mathbf{p}_{3} \wedge \mathbf{k}\right)}{\left(\mathbf{p}_{1}-\mathbf{k}\right)^{2}\left(\mathbf{p}_{3}-\mathbf{k}\right)^{2}} I_{k^{0}}
$$

com

$$
I_{k 0}=\int \frac{d k^{0}}{(2 \pi)} \Delta(k) \Delta\left(p_{1}+p_{3}+k\right)=\frac{-i m}{\mathbf{p}^{2}-\mathbf{k}^{2}+i \epsilon},
$$

sendo que a integração em $k^{0}$ foi feita usando o teorema dos resíduos e na concha de massa. Assim

$$
\mathcal{M}_{b}(\theta)=-\frac{4 g^{4}}{m \Theta^{2}}\left[T^{\mathrm{c}} T^{a} \otimes T_{c} T_{a}\right] \int \frac{d^{2} \mathbf{k}}{(2 \pi)^{2}} \frac{\left(\mathbf{p}_{1} \wedge \mathbf{k}\right)\left(\mathbf{p}_{3} \wedge \mathbf{k}\right)}{\left(\mathbf{p}_{1}-\mathbf{k}\right)^{2}\left(\mathbf{p}_{3}-\mathbf{k}\right)^{2}\left[\mathbf{p}^{2}-\mathbf{k}^{2}+i \epsilon\right]} .
$$

Conforme a figura (B.1). e equações (B.9)-(B.12), podemos fazer a integração angular, obtendo

$$
\mathcal{M}_{b}(\theta)=-\frac{g^{4}}{4 \pi^{2} m \Theta^{2}}\left[T^{c} T^{a} \otimes T_{c} T_{a}\right] \int_{0}^{A N_{R}} d(\mathbf{k})^{2} \frac{1}{\mathbf{p}^{2}-\mathbf{k}^{2}+i \epsilon}\left[\cos \theta I_{0}-I_{2}\right],
$$

ou seja

$$
\begin{aligned}
\mathcal{M}_{b}(\theta)= & -\frac{g^{4}}{4 \pi^{2} m \Theta^{2}}\left[T^{c} T^{a} \otimes T_{c} T_{a}\right] \int_{0}^{i_{N R}} d(\mathbf{k})^{2}\left\{\frac{1}{\mathbf{p}^{2}-\mathbf{k}^{2}+i \epsilon}\right. \\
& \left.+S g\left(\mathbf{k}^{2}-\mathbf{p}^{2}\right) \frac{\mathbf{k}^{2}+\mathbf{p}^{2}}{\mathbf{k}^{4}+\mathbf{p}^{4}-2 \mathbf{k}^{2} \mathbf{p}^{2} \cos \theta}\right\} .
\end{aligned}
$$


Usando as equaçóes (B.14) a (B.16) obtemos o resultado (2.20).

- Gráfico (2.11.c4)

Analiticamente o gráfico (2.11.e4) é dado por

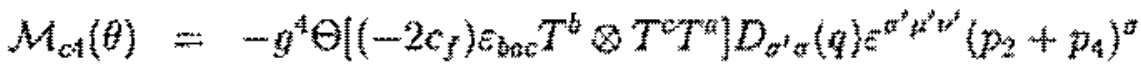

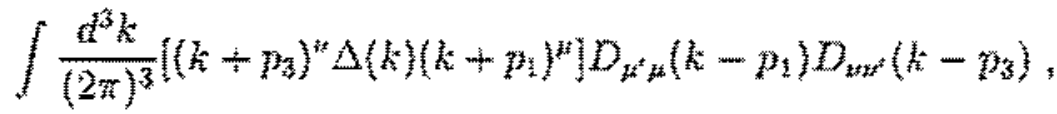

onde q é o momento transferido. Lsando as regras de Feynman podemos escrever

$$
M_{c 4}(\theta)=\frac{-g^{4}}{\theta^{2}}\left[\left(-2 c_{j}\right) \varepsilon_{b a c} T^{b} \otimes T^{c} T^{a}\right] \int \frac{d^{3} k}{(2 m)^{3}} \frac{N_{a}}{\left.\left[k^{2}-m^{2}+i t\right]\left(k-\mathbf{p}_{1}\right)^{2}\right]\left[\left(\mathrm{k}-\mathrm{p}_{3}\right)^{2}\right]\left[\mathrm{q}^{2}\right]^{2}}
$$

com

$$
N_{c i}=\left[\mathrm{q} \wedge \mathrm{k}-\mathrm{p}_{1} \wedge \mathrm{p}_{3}\right]\left\{2 w_{q}\left(k^{0}+u_{q}\right][2 \mathrm{q} \wedge \mathrm{k}]+\left(k^{0}+w_{q}\right)^{2}\left[2 \mathrm{p}_{1} \wedge \mathrm{p}_{3}\right]\right\},
$$

e $u_{q}=\sqrt{q^{2}+m^{2}}$. Separando a integraçäo em $k^{0}$

$$
\mathcal{M}_{c 4}(\theta)=\frac{-2 g^{4}}{\Theta^{2}}\left[\left(-2 c_{f}\right) \varepsilon_{b c c} T^{b} \otimes T^{2} T^{a}\right] \int \frac{d^{2} \mathrm{k}}{(2 \pi)^{2}} \frac{\left[\mathbf{q} \wedge \mathrm{k} \cdots \mathrm{p}_{\mathrm{l}} \wedge \mathrm{p}_{3}\right]}{\left[\left(\mathbf{k}-\mathbf{p}_{1}\right)^{2}\right]\left[\left(\mathbf{k}-\mathbf{p}_{3}\right)^{2}\right]\left[\mathbf{q}^{2}\right]} T_{0}
$$

com

$$
T_{0}=\int \frac{d k^{0}}{(2 \pi)} \frac{2 w_{q}\left(k^{0}+w_{q}\right)[\mathbf{q} \wedge \mathbf{k}]+\left(k^{0}+w_{q}\right)^{2}\left[\mathbf{p}_{1} \wedge \mathbf{p}_{3}\right]}{k_{0}^{2}-w_{k}^{2}+i k}
$$

A integração em $k^{0}$ tem problema de divergência, por isso precisamos usar algum tipo de regularização para podermos calcular a integral. Os termos ímpares en $k_{0}$ săo nulos por simetria de integracăo. Coloxando $u$ m regulador no limite de integração $\left(-1<k_{n}<1\right)$, obtemos podemos escrever $T_{\mathrm{B}}$ como

$$
T_{0}=[\mathbf{q} \wedge \mathbf{k}] \frac{w_{p}^{2}}{w_{k}}+\left[\mathbf{p}_{1} \wedge \mathbf{p}_{3}\right] \frac{u_{p}^{2}+u_{k}^{2}}{2 u_{k}}+\frac{l}{\pi}\left[\mathbf{p}_{1} \wedge \mathbf{p}_{3}\right],
$$

onde temos usado $\left|\mathrm{p}_{1}\right|=\left|\mathrm{p}_{3}\right|=|\mathrm{p}| .0$ termo dependente de $1 \mathrm{e}$

$$
M_{c 4}^{T} \times \frac{l}{\pi}\left[\mathrm{p}_{1} \wedge \mathrm{p}_{3}\right] \int \frac{d^{2} \mathrm{k}}{(2 \pi)^{2}} \frac{\left[\mathrm{q} A \mathrm{k}-\mathrm{p}_{1} \wedge \mathrm{p}_{3}\right]}{\left(\left(\mathrm{k}-\mathrm{p}_{1}\right)^{2}\right]\left[\left(\mathrm{k}-\mathrm{p}_{3}\right)^{2}\right]},
$$

porém a integraçào espacial na equaçăo acima é identicamente nula. Isto pode ser fácilmente visto se usarmos a parametrizagăo de Feynman e fazendo um deslocamento na 
variável de integração. Assim, substituindo (C.11) em (C.9) podemos separar a integração angular, isto é,

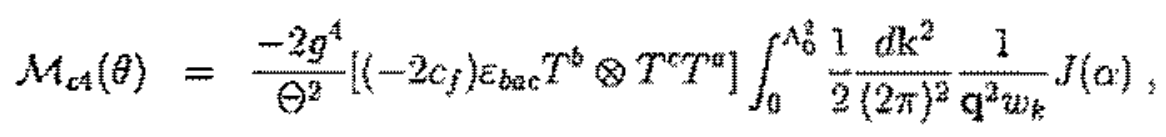

onde

$$
J(\alpha)=\int_{0}^{2 \pi} d \alpha \frac{\left[\mathrm{q} \wedge \mathrm{k}-\mathrm{p}_{1} \wedge \mathrm{p}_{3}\right]}{\left[\left(\mathrm{k}-\mathrm{p}_{1}\right)^{2}\right]\left[\left(\mathrm{k}-\mathrm{p}_{3}\right)^{2}\right]}\left\{[\mathrm{q} \wedge \mathrm{k}] \frac{w_{z}^{2}}{w_{k}}+\left[\mathrm{p}_{1} \wedge \mathrm{p}_{3}\right] \frac{w_{\mathrm{p}}^{2}+w_{k}^{2}}{2 w_{k}}\right\}
$$

De acordo com a figura (B.1), e equaçôes (B.9)-(B.12), podemos escrever a integraçào angular na forma

$$
\begin{aligned}
J(\alpha) & =\left[2 e_{p}^{2}(1-\cos \theta)\left[I_{0}+I_{2}\right]+\frac{\mathrm{p}^{2}}{|\mathrm{p} \| \mathrm{k}|} \sin \theta\left(u_{k}^{2}-u_{p}^{2}\right) 2 \sin \theta / 2 I_{1}\right. \\
& \left.-\frac{\mathrm{p}^{2}}{\mathrm{k}^{2}} \sin ^{2} \theta\left(u_{p}^{2}+w_{k}^{2}\right) I_{0}\right] .
\end{aligned}
$$

Assim, podemos escrever, após algumas manipulaçöes, que

$$
\begin{array}{r}
J(\alpha)=2 \pi(1-\cos \theta)\left\{w_{p}^{2}+\sin a l\left(\mathbf{k}^{2}-\mathrm{p}^{2}\right) \times\left[\frac{-\left(\mathrm{p}^{2}+m^{2}\right) \mathbf{k}^{4}}{\mathrm{k}^{4}+\mathrm{p}^{4}-2 \mathrm{p}^{2} \mathrm{k}^{2} \cos \theta}\right.\right. \\
\left.\left.+\frac{\left.\left.\left(3 \mathrm{p}^{2}+2 m^{2}\right) \mathrm{p}^{2}(1-\cos \theta) \mathbf{k}^{2}+\left[(2+\cos \theta) \mathrm{p}^{6}+(3+2 \cos \theta) m^{2} \mathrm{p}^{4}\right]\right]\right\} .}{\mathrm{k}^{4}+\mathrm{p}^{4}-2 \mathrm{p}^{2} \mathrm{k}^{2} \cos \theta}\right]\right\}
\end{array}
$$

Substituindo (C.16) em (C.13) e fazendo a separaçäo na parte de alta e baixa energia, como descrito no capítulo 2, nós obtemos os resultados (2.99) e (2.100). 


\section{Apêndice D}

\section{Espalhamento em 1-Loop (Spin 1/2)}

Neste apêndice vamos explicitar as contas para o gráfico (3.81). Este gráfico que contém o vêrtice corn trễs gluons e é dado analiticamente por festamos ornitindos os indices externos)

$$
\begin{aligned}
& M_{d}=\int \frac{d^{3} k}{(2 \pi)^{3}}\left\{\left[\bar{u}\left(p_{4}\right) \Gamma^{b, p} u\left(p_{2}\right)\right] D_{z \mu}^{b a}(g) \Gamma^{a c^{\prime} f^{\prime}, \alpha \rho \sigma} D_{\sigma \alpha}^{d^{\prime} d}\left(k-p_{3}\right)\right. \\
& \left.\times D_{s_{p}}^{c u^{\prime}}\left(k-p_{1}\right)\left[\bar{u}\left(p_{3}\right) \Gamma^{d_{1} \wedge} S(k) \Gamma^{c_{1}, 3} u\left(p_{1}\right)\right]\right\} \\
& =\frac{i(-g)^{3}}{(\Theta)^{3}}\left[-2 i c_{f} g \Theta \varepsilon^{n c d} T_{a} \otimes T_{d} T_{c}\right] \int \frac{d^{3} k}{(2 \pi)^{3}} \frac{N_{d}}{\left[k^{2}-m^{2}+i \epsilon\right]\left(\mathrm{k}-\mathrm{p}_{1}\right)^{2}\left(\mathrm{k}-\mathrm{p}_{3}\right)^{2} \mathrm{q}^{2}}
\end{aligned}
$$

com

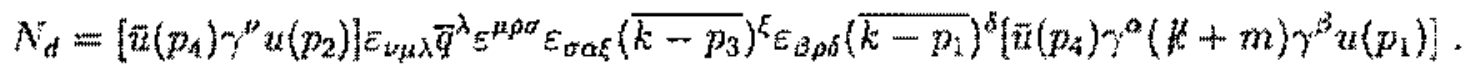

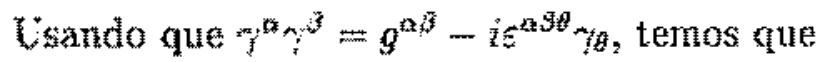

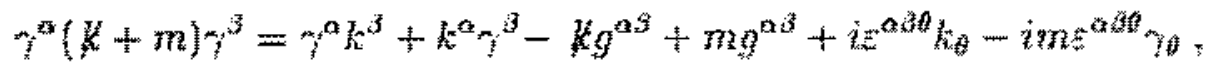

$\log 0$

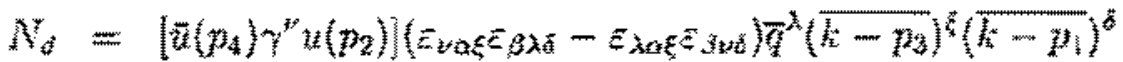

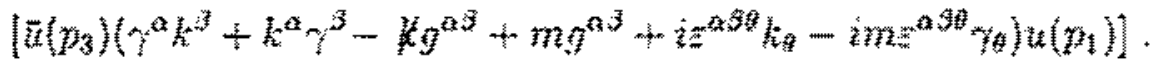

Abrindo as componentes tembrando que os temos ímpares em $k^{0}$ săo nulos pela simetria na integraçăo em $k^{0}$, temos

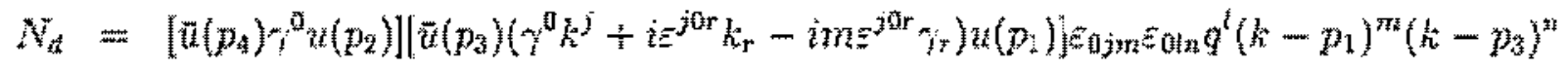




$$
\begin{aligned}
& \text { - }\left[\ddot{u}\left(p_{i}\right) \gamma^{0} u\left(p_{2}\right)\right]\left[\tilde{u}\left(p_{3}\right)\left(\gamma^{0} k^{j}+i \varepsilon^{0 j r} k_{\tau}-i m \varepsilon^{0 j r} \gamma_{\tau}\right) u\left(p_{1}\right)\right] \varepsilon_{i 0 m} \varepsilon_{j 0 n} q^{l}\left(k-p_{1}\right)^{n}\left(k-p_{3}\right]^{n} \\
& +\left[\bar{u}\left(p_{4}\right) \gamma^{i} u\left(p_{2}\right)\right]\left[\vec{u}\left(p_{3}\right)\left(k_{j} \gamma^{j}+m\right) u\left(p_{1}\right)\right]\left(\varepsilon_{i m n} \varepsilon_{0 i m}-\varepsilon_{10 n} \varepsilon_{0 i m}\right) q^{t}\left(k-p_{1}\right)^{m}\left(k-p_{3}\right)^{n}
\end{aligned}
$$

usando que $\mathrm{atg}_{\mathrm{a}} \mathrm{a}^{\mathrm{i}} \mathrm{b}=\mathrm{a} \mathrm{A} \mathrm{b}$ podenos escrever

$$
\begin{aligned}
& N_{d}=\left\{\left[u\left(p_{4}\right) \gamma^{0} u\left(p_{2}\right)\right]\left[\bar{u}\left(p_{3}\right)\left(\gamma^{0} k^{j}+i \varepsilon^{j 0 r} k_{r}-i m \varepsilon^{j 0 *} \gamma\right) u\left(p_{1}\right)\right] \varepsilon_{0 j m}\left(k-p_{1}\right)^{m}\right.
\end{aligned}
$$

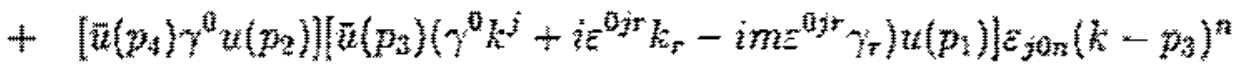

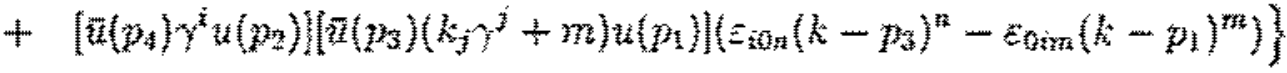

$$
\begin{aligned}
& x_{[}\left[\mathrm{a} \wedge \mathrm{k}-\mathrm{p}_{1} \wedge \mathrm{p}_{\mathrm{s}}\right] \text {. }
\end{aligned}
$$

Temos que até ordem $\mathrm{p} / \mathrm{m}$

$$
\left[u\left(p^{n}\right) \gamma^{0} u(p)\right]=\left[\bar{u}\left(p^{i}\right) u(p)\right]=1, \quad\left[\bar{u}\left(p^{\prime}\right) \eta^{i} u(p)\right]=\frac{1}{2 m n}\left[P^{i}+i \varepsilon^{i j} q^{3}\right]
$$

onde $P^{i}=p^{z}+p^{t i}$ e $q^{i}=p^{i}-p^{i}$. Isso nos leva a

$$
N_{d}=\left[\mathrm{q} \wedge \mathrm{k}-\mathrm{p}_{1} \wedge \mathrm{p}_{3}\right]\left\{2 i\left[\mathrm{k}^{2}+\mathrm{p}^{2}\right]-2[\mathrm{q} \wedge \mathrm{k}+i \mathrm{P} \cdot \mathrm{k}]\right\} .
$$

Substituindo na equaçăo (D.1) temos

$$
\begin{aligned}
M_{d}= & \frac{-2 g^{4}}{\Theta^{2}}\left[c_{f} \Theta z^{a c d} T_{a} \otimes T_{d} T_{c}\right] \int \frac{d^{2} \mathbf{k}}{(2 \pi)^{2}} \frac{\left[\mathbf{q} \wedge \mathbf{k}-\mathbf{p}_{1} \wedge \mathbf{p}_{3}\right]}{\left(\mathbf{k}-\mathbf{p}_{1}\right)^{2}\left(\mathbf{k}-\mathbf{p}_{3}\right)^{2} \mathbf{q}^{2}} \\
& \left\{2 i\left[k^{2}+\mathbf{p}^{2}\right]-2[\mathbf{q} \wedge \mathbf{k}+i \mathbf{P} . \mathbf{k}]\right\} I_{k^{0}}
\end{aligned}
$$

na qual

$$
I_{k a}=\int \frac{d k^{0}}{2 \pi} \frac{1}{\left[k_{j}^{2}-\psi_{i}^{2}+i t\right]}=-\frac{i}{2 u_{k}},
$$

con $w_{k}^{2}=\mathrm{k}^{2}+m^{2}$. Separando a parte angular na integraçăo espacial

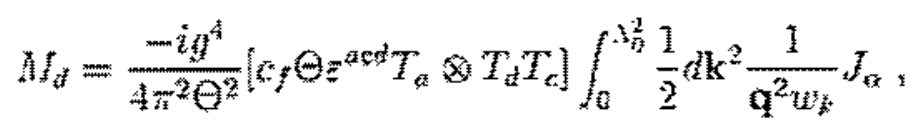

sendo

$$
J_{a}=\int_{0}^{3=} d a \frac{\left[\mathrm{q} \wedge \mathrm{k}-\mathrm{p}_{1} \wedge \mathrm{p}_{3}\right]\left[2[\mathrm{q} \wedge \mathrm{k}+\mathrm{iP} \cdot \mathrm{k}]-2 i\left[\mathrm{k}^{2}+\mathrm{p}^{2}\right]\right\}}{\left(\mathrm{k}-\mathrm{p}_{1}\right)^{2}\left(\mathrm{k}-\mathrm{p}_{3}\right)^{2}}
$$

De acordo con a figura (B.1), do apendice B, podemos escrever,

$$
\left[q \wedge \mathrm{k}-\mathrm{p}_{1} \wedge \mathrm{p}_{3}\right]=\left[2|\mathrm{p}||\mathrm{k}| \sin (\theta / 2) \cos (\alpha)-\mathrm{p}^{2} \sin (\theta)\right]
$$


e sambém que

$$
\begin{aligned}
& \left.\left[\mathbf{q} \wedge k-p_{1} \wedge p_{3}\right]\left\{2[a \wedge k+i p . k]-2 i k^{2}+p^{2}\right]\right\}= \\
& =2\left[\mathbf{p}|\mathbf{k}|\left[2 \sin ^{2}(\theta / 2)+2 \sin (\theta / 2) \cos (\theta / 2)\right]\right. \\
& \left.\times\left[|p| k \mid(1+\cos 2(\alpha))-2 p^{2} \cos (\theta) 2\right) \cos (\alpha)\right]
\end{aligned}
$$

para o denominador temos

$$
\begin{aligned}
& \left.\left(\mathbf{k}-\mathbf{p}_{1}\right)^{2}=|\mathbf{p}| \mathbf{k} \mid \mathbf{2} \cos (\alpha+6 / 2)-f\right] \\
& \left(\mathbf{k}-\mathbf{p}_{3}\right)^{2}=|\mathrm{p}| \mathbf{k} \mid(2 \cos (\alpha-6 / 2)-f]
\end{aligned}
$$

Assim, podemos escrever, con o uso da relaçấo $(\mathrm{B} .8)$, $(\mathrm{B} .9)$, que

$$
\begin{aligned}
J_{a}= & \frac{2}{\mathbf{p}^{2} \mathbf{k}^{2}}(1-\cos \theta+i \sin \theta)\left\{|\mathbf{p}||\mathbf{k}|\left(I_{0}+I_{2}\right)-2 \mathbf{p}^{2} \cos (\theta / 2) I_{1}\right) \\
& -2 i \frac{\mathbf{k}^{2}+\mathbf{p}^{2}}{\mathbf{p}^{2} \mathbf{k}^{2}}\left[2|\mathbf{p}||\mathbf{k}| \sin (\theta / 2) I_{1}-\mathrm{p}^{2} \sin (\theta) I_{0}\right)
\end{aligned}
$$

ou ainda

$$
\begin{aligned}
J_{\alpha}= & 2 \pi\left\{[(1-\cos \theta)+i \sin \theta]\left[1-\sin a l\left(\mathbf{k}^{2}-\mathrm{p}^{2}\right)\right\}\right. \\
& \left.+\operatorname{sinal}\left(\mathrm{k}^{2}-\mathbf{p}^{2}\right)\left[2 \mathrm{p}^{2}(1-\cos \theta) \frac{\mathrm{k}^{2}+\mathbf{p}^{2}}{R}\right]\right\}
\end{aligned}
$$

onde $R=\left(k^{4}+p^{4}-2 p^{2} k^{2} \cos \theta\right)$. Substituindo (D.17) em (D.11) temos

$$
H_{d}=\frac{-i g^{4}}{4 \pi \theta^{2}}\left[c_{i} \Theta \varepsilon^{a c d} T_{t} \otimes T_{d} T_{t}\right] T_{k}
$$

com

$$
\begin{aligned}
& T_{k}=\int_{0}^{12} \frac{d k^{2}}{q^{2} w_{k}}[(1-\cos \theta)+i \sin \theta] !-5 q\left(k^{2}-p^{2}\right) \\
& \left.+5 q\left(k^{2}-p^{2}\right)\left[2 p^{2}(1-\cos \theta) \frac{k^{2}+p^{2}}{R}\right]\right]
\end{aligned}
$$

Vamos separar a integral em $\mathrm{k}^{2}$ na parte de baixa energia (L) e alta energia (H). Na parte de baixa energita fazemos uma expansäo en $1 / m$.

$$
\frac{1}{2 m_{m}}=\frac{1}{m}\left(1-\frac{k^{2}}{2 m^{2}}+\ldots\right)
$$


Na parte de alta energia fazemos uma expansão em torno de $p=0$. Entăo

$$
\frac{1}{R}=\frac{1}{k^{4}}\left(1+\frac{2 \mathrm{p}^{2}}{\mathrm{k}^{2}} \cos \theta+\ldots\right) .
$$

Assin, usando as equaçoses (B.14) - (B.18), obtemos para a parte de baixa energia, temos até ordem $\eta$

$$
\begin{aligned}
M_{a}^{L}= & \frac{-i g^{4}}{4 \pi m \Theta^{2}}\left[c_{f} \Theta \varepsilon^{a c d} T_{a} \otimes T_{t} T_{c}\right]\left\{1+i \frac{\sin \theta}{1-\cos \theta}\right. \\
& \left.+\left[\log \left(\frac{\Lambda^{2}}{q^{2}}\right)-\frac{\Lambda^{2}}{2 m^{2}}-(1+2 \cos \theta) \frac{p^{2}}{\Lambda^{2}}\right]\right\} .
\end{aligned}
$$

e para a parte de alta energia, con a ajuda da equaçoes (B.10) a (B.22)

$$
M_{d}^{H}=\frac{-i g^{4}}{4 \pi m \theta^{2}}\left[c_{f} \Theta \varepsilon^{a c d} T_{a} \otimes T_{d} T_{c}\right]\left\{-\log \left(\frac{A^{2}}{4 m^{2}}\right)+\frac{A^{2}}{2 m^{2}}+(1+2 \cos \theta) \frac{p^{2}}{\lambda^{2}}\right\} .
$$

Somando a contribuição das equagōes $\{0.20\}$ e $\{0.21\}$ obtemos

$$
M_{d}=\frac{-i g^{4}}{4 \pi m \Theta^{2}}\left[c j \Theta \varepsilon^{a c t} T_{n} T_{d} T_{d}\right]\left\{1+\frac{\sin \theta}{1-\cos \theta}+\log \left(\frac{4 m^{2}}{\mathrm{q}^{2}}\right)\right\} .
$$




\section{Apêndice E}

\section{Cálculos em 1-Loop (Spin 1)}

\section{E.1 Auto-Energia}

A contribuiçào para a auto-energia do méson vem do gráfico (2.3.a), que analiticamente é dado por

$$
\Sigma^{\alpha, 3}(p)=\int \frac{d^{3} k}{(2 \pi)^{3}} \Gamma_{1}^{\nu \alpha \alpha^{\prime}}(p, p+k) \Delta_{\alpha^{\prime} B}(p+k) \Gamma_{1}^{\mu 3^{\prime} 3}(p+k, p) D_{\mu, \nu}(k) .
$$

Usando que a parametrizaçāo de Feynman

$$
\frac{1}{A B}=\int_{0}^{1} d x \frac{1}{[(A-B) x+B]^{2}},
$$

e fazendo a translação $k \rightarrow k+p x$, podemos escrever

$$
\Sigma^{\alpha 3}(p)=\frac{-i e^{2}}{\theta} \int_{0}^{1} d x \int \frac{d^{3} k}{(2 \pi)^{3}} \frac{N_{S}^{\alpha 3}}{\left[k^{2}-C^{2}\right]}
$$

onde $C^{2}=M^{2}(1-x)-p^{2} x(1-x) \mathrm{e}$

$$
\begin{aligned}
N_{S}^{\alpha 3} & =\left\{\left[3 M^{2}+x p^{2}\right]\left(\varepsilon^{\sigma \beta \rho} k_{\sigma} k^{\alpha}+\Xi^{\alpha \sigma \rho} k_{\sigma} k^{\beta}\right) p_{\rho}+\left[(1-x) M^{2} p^{2}\right.\right. \\
& \left.\left.-(1-x)\left(p_{\mu} k^{\mu}\right)^{2}\left(x^{2}-x^{3}\right) p^{4}\right] \varepsilon^{\alpha 3 \sigma} p_{\sigma}+2 x p^{2} \Xi^{\alpha \beta \rho} k_{\rho} k_{\sigma} p^{\sigma}\right\} / M^{2} .
\end{aligned}
$$

Através do uso da regularizaçāo dimensional, obtemos

$$
\Sigma^{\alpha 3}(p)=\frac{e^{2}}{8 \pi \theta M^{2}} \int_{0}^{1} d x \frac{6 M^{4}+\left[p^{4}-M^{2} p^{2}-6 M^{4}\right] x+\left[M^{2} p^{2}-7 p^{4}\right] x^{2}+6 p^{4} x^{3}}{\sqrt{M^{2}(1-x)-p^{2} x(1-x)}} \epsilon^{\alpha 3 \sigma} p_{\sigma}
$$


Após efetuarmos a integração paramétrica

$$
\Sigma^{\alpha \beta}(p)=\frac{e^{2}}{8 \pi \theta} F(p) \varepsilon^{\alpha \beta \rho} p_{\rho}
$$

onde

$$
F(p)=\frac{\left(3 M^{2}+p^{2}\right)}{2 M p^{2}}\left[\left(M^{2}+p^{2}\right)-\frac{1}{2 \sqrt{p^{2}}}\left(M^{2}-p^{2}\right)^{2} \log \left(\frac{\sqrt{M^{2}}+\sqrt{p^{2}}}{\sqrt{M^{2}}-\sqrt{p^{2}}}\right)\right] .
$$

\section{E.2 Polarização}

A contribuição para o tensor de polarização ven dos gráficos da figura (2.4)

$$
\Pi^{\mu \prime \prime}=\Pi_{A}^{\mu \nu}+\Pi_{B}^{\mu \nu}
$$

com

$$
\begin{aligned}
\Pi_{A}^{\mu \nu} & =\int \frac{d^{3} k}{(2 \pi)^{3}} \Gamma_{1}^{\mu j a}(p+k, k) \Delta_{\alpha \alpha^{\prime}}(k) \Gamma_{1}^{\nu \alpha^{\prime} B^{\prime}}(k, p+k) \Delta_{\beta^{\prime} \beta}(p+k) \\
\Pi_{B}^{\mu \nu} & =\int \frac{d^{3} k}{(2 \pi)^{3}} \Gamma_{2}^{\mu \nu \alpha 3} \Delta_{\alpha 3}(k) .
\end{aligned}
$$

Usando as regras de Feynman podemos escrever

$$
\Pi^{\mu \prime \prime}=\frac{e^{2}}{M^{2}} \int \frac{d^{3} k}{(2 \pi)^{3}} \frac{N_{P l}^{\mu \prime}}{\left[k^{2}-M^{2}\right]\left[(k+p)^{2}-M^{2}\right]},
$$

onde

$$
\begin{aligned}
N_{P 1}^{\mu \nu} & =\left[k^{2}\left(p^{\mu} k^{\nu}+k^{\mu} p^{\mu}\right)-2 k^{\mu} k^{\nu} k^{\circ} p_{\mathrm{o}}\right]+\left[8 M^{2} k^{\mu} k^{\nu}-\left(4 M^{2} g^{\mu \nu}+p^{\mu} p^{\nu}\right) k^{2}\right. \\
& \left.+k^{\alpha} p_{\alpha}\left(2 p^{\mu} k^{\nu}+2 k^{\mu} p^{\nu}-2 g^{\mu \nu} k^{\beta} p_{\beta}\right)+p^{2}\left(-3 k^{\mu} k^{\nu}+2 g^{\mu \nu} k^{2}\right)\right] \\
& +\left[3 M^{2}\left(p^{\mu \prime} k^{\prime \prime}+k^{\mu} p^{\nu}-6 g^{\mu \nu} k^{\alpha} p_{\mathrm{o}}\right)\right]+\left[M^{2}\left(4 M^{2} g^{\mu \nu}+p^{\mu} p^{\nu}-3 p^{2} g^{\mu \nu}\right)\right]
\end{aligned}
$$

Usando (E.2) depois fazendo um translaçāo $k \rightarrow k-p x$ e eliminando os termos ímpares em $k$,

$$
\Pi^{\mu \nu}=\frac{e^{2}}{M^{2}} \int_{0}^{1} d x \int \frac{d^{3} k}{(2 \pi)^{3}} \frac{N_{P 2}^{\mu \prime \prime}}{\left[k^{2}-a^{2}\right]},
$$

onde $a^{2}=M^{2}-p^{2} x(1-x) \mathrm{e}$

$$
\begin{aligned}
N_{P 2}^{\mu \nu} & =M^{2}\left[4 M^{2} g^{\mu \prime}+p^{\mu} p^{\nu}\left(1-6 x+8 x^{2}\right)+p^{2} g^{\mu \prime \prime}\left(-3+6 x-4 x^{2}\right)\right] \\
& +k^{\mu} k^{\nu}\left[8 M^{2}-3 p^{2}+2 p^{2} x\right]+k^{2}\left[-4 M^{2} g^{\mu \prime}-p^{\mu} p^{\nu}(1+2 x)+2 p^{2} g^{\mu \nu}\right] \\
& +\left[2 k^{\mu} p^{\nu} k^{\alpha} p_{\alpha}+2 p^{\mu} k^{\nu} k^{\alpha} p_{\alpha}-2 g^{\mu \prime \prime}\left(k^{\alpha} p_{\alpha}\right)^{2}\right] .
\end{aligned}
$$


Novamente para a integração no momento interno vamos usar a regularização dimensional. Deste modo temos

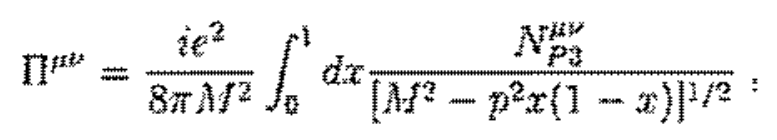

com

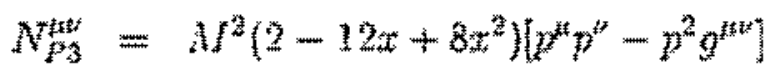

$$
\begin{aligned}
& +p^{2}\left[p^{3} p^{4}\left(-x+7 x^{2}-6 x^{3}\right)+p^{2} g^{t y}\left(-x-x^{2}+2 x^{3}\right)\right]
\end{aligned}
$$

Efetuando a integraçă em $x$ obtemos

$$
\Pi^{\mu t}=\frac{i e^{2}}{8 \pi} \Pi\left(p^{2}\right)\left(g^{i t}-\frac{p^{4} p^{y}}{p^{2}}\right)
$$

$\operatorname{com} \Pi\left(p^{2}\right)$ dado por

$$
\Pi\left(p^{2}\right)=\frac{\left(p^{2}-4 M^{2}\right)}{4 M^{2}}\left[4 \sqrt{M^{2}}-\left(p^{2}+4 M^{2}\right) I_{0}\right]
$$

onde

$$
I_{0}=\int_{0}^{1} d x \frac{1}{\sqrt{M^{2}-p^{2} x(1-x)}}=\frac{1}{\sqrt{p^{2}}} \operatorname{Ln}\left[\frac{2 \sqrt{M^{2} p^{2}}+y^{2}}{2 \sqrt{M^{2} p^{2}}-p^{2}}\right] .
$$

\section{E.3 Espalhamento}

O gräfico (2.6.4) analiticamente é dado por

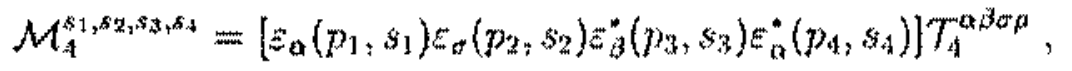

onde

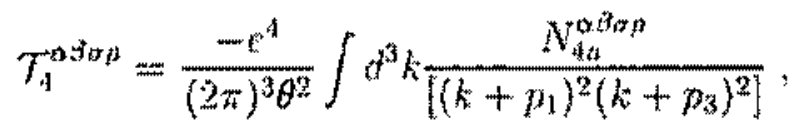

com

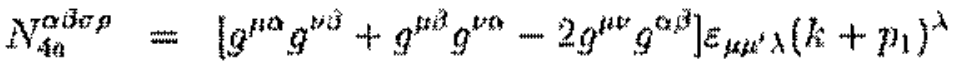

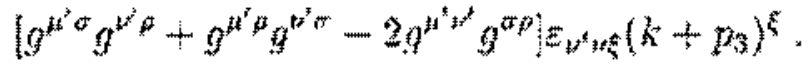

Na concha de massa e usando a equaçäo (E.2) podemos, após uma translaçäo $k \rightarrow k-$ $p_{1}, t-p_{\mathfrak{d}}(1-x)$, escrever $(E .20)$ como

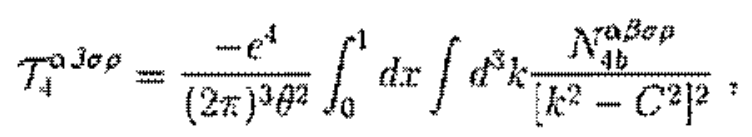




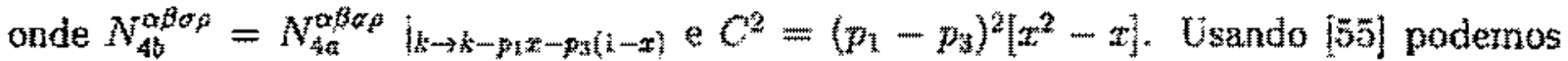
integrar em $k$ e posteriormente em $x$. Substituindo esse resultado am (E.19) e fazendo as contraçōes com as polarizaçöes externas obtemos'

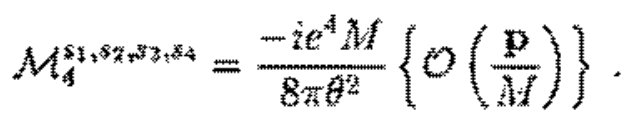

O gráfico (2.6.5) usando-se as regras te Feynman é dado por

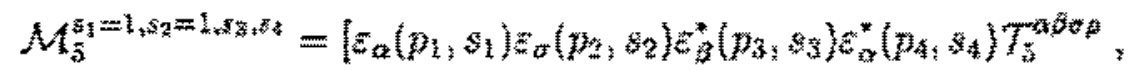

no qual

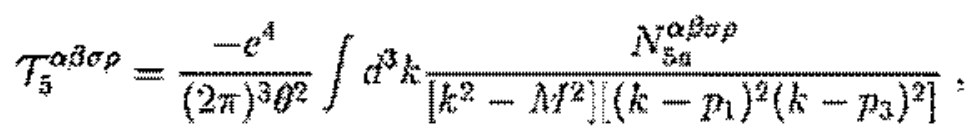

$\mathrm{e}$

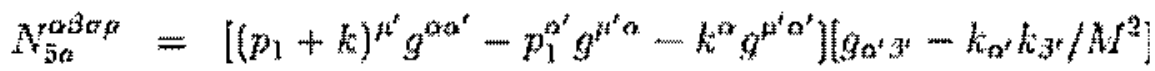

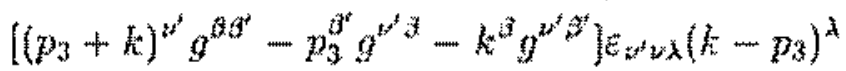

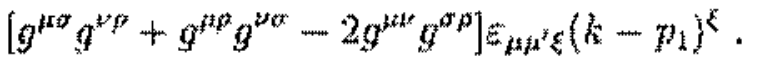

Com o auxilio da parametrizaça (4.64) podenos escrever (E.25), na concha de massa, como

$$
\mathcal{T}_{5}^{\mathrm{a} B \theta \rho}=\frac{-e^{4}}{(2 \pi)^{3} \theta^{2}} \int_{0}^{1} d x \int_{0}^{1} d y 2 y \int d^{3} k \frac{N_{s t}^{\alpha \beta z p}}{\left[k^{2}-C^{2}\right]^{3}}
$$

onde $N_{5 b}^{\alpha \beta a p}=\left.N_{b a}^{\alpha \beta a \rho}\right|_{k \rightarrow k+l y}, l=p_{3}(1-x)+p_{1} x$ e $C^{2}=M^{2}(1-y)+l^{2} y^{2}$. Integrando em $k$ com o auxílio de [55] e fazendo aproximaçăo até ordem $\mathfrak{p} / M$ tal que $C^{3} \approx M^{2}\left[1-y+y^{2}\right]$, podemos fazer a integração em $x$ e posteriormente a integração em $y$. Substituindo esse resultado en (E.24) e fazendo a contração com as polarizaçoes externas obtemos, na orden dominante em $\mathrm{p} / \mathrm{M}$,

$$
\begin{aligned}
& M_{\bar{\delta}}^{s_{1}=1, s_{z}=1 . s_{3}, s_{4}}=\frac{-i e^{4} M}{8 \pi \theta^{2}}(\cos 0-i \sin \alpha)\left\{\frac{\left(1+s_{3}\right)\left(1+s_{3}\right)}{64}\right. \\
& [-28+55 \ln (3)](\cos \alpha-i \sin \alpha)\} \\
& M_{3}^{s_{1}=-s_{1} s_{2}=-i, s_{3}, b_{4}}=\frac{-i e^{4} M}{8 \pi \theta^{2}}(\cos a+i \sin \alpha)\left\{\frac{\left(1-s_{3}\right)\left(1-s_{4}\right)}{64}\right. \\
& [-28+5 \ln (3)](\cos \alpha+i \sin \alpha)\}
\end{aligned}
$$

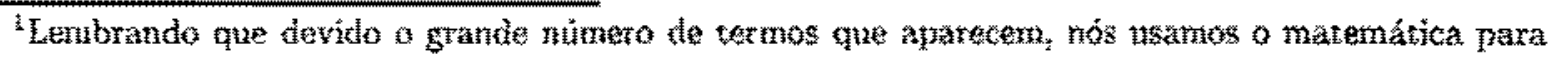
integraçôes e contragös censoriais. 


$$
\begin{aligned}
& \mathcal{M}_{5}^{s_{1}=m, z_{2}=-1, s_{3}, s_{4}}=\frac{-\dot{x} e^{4} M}{8 \pi \theta^{2}}\left(\left(-1+s_{3} s_{4}\right)[-36+73 \ln (3)]\right. \\
& \left.+\left(s_{3}-s_{4}\right)\{-20+37 \ln (3)]\right\} \\
& M_{s}^{s_{1}=-1, s_{2}=1, s_{3}, s_{4}}=\frac{-i e^{4} M}{8 \pi \theta^{2}}\left\{\left(1-s_{s} s_{4}\right)[-36+73 \ln (3\}]\right. \\
& \left.\left.-\left(s_{3}-s_{i}\right) \mid-20+37 \ln (3)\right]\right\} \text {. }
\end{aligned}
$$

O gráfico (25.6) é feito de modo semelhante. 


\section{Bibliografia}

[1] J. F. Schonfeld, Nucl. Phys. B185 (1981) 157: S. Deser, R. Jackiw e S. Templeton, Ann. Phys. (N.Y.) 140 (1982) 372: R. Jackiw and S. Templeton, Phys. Rev D23 (1981) 2291;

[2] R. B. Laughlin, Phys. Rer. B39 (1989) 9679; Y.-H. Chen, F. Wilczek, E. Witten and B. Halperin, Int. J. Mod. Phys. B3 (1989) 1001; The Quantum Hall Effect, edited by R. Prange and S. Girvin (Springer, Berlim,1990).

[3] P. B. Wiegmann, Phys. Rev. Lett. 60 (1988) 821: F. Wilczek, Fractional Statistics and Anyon Superconductivity, Word Scientific, Singapore (1990); A. P. Balachandran, G. Landi, B. Rai, Int. J. Mod. Phys. A5 (1990) 4501; A. Zee, $25^{\circ}$ Int. Conf. on High Energy Physics, Singapore (1990). Published in Singapore H. E. Phys. 1990:206-212, Ed. K. K. Phua and Y. Yamaguchi.

[4] H. O. Girotti, M. Gomes e A. J. da Silva, Niod. Phys. 29 (1994) 2699.

[5] F. Wilczek, Phys. Rev. Lett. 48 (1982) 1144: J. M. Leinass and J. Myrhein, Il Nouvo Cimento 37 (1977) 1.

[6] C. R. Hagen, Ann. Phys. (N.Y.) 140 (1984) 342, Phys. Rev. D 31 (1985) 2135.

[7] D. P. Arovas, J. R. Schrieffer and F. Wilczek. Phys. Rev. Lett.53 (1984) 772.

[8] Aharonov and Bohm, Phys. Rev. 115 (1959) 485.

[9] P. K. Townsend, K. Pilch and P. Van Nieuwenhuizen, Phys. Lett. B136 (1984) 38:

[10] S. Deser, R. Jackiw, Phys. Lett. B139 (1984) 371.

[11] R. Banerjee, H. J. Rothe and K. D. Rothe, Phỵs. Rev. D52 (1995) 3750.

[12] I. A. Batalin and E. S. Fradkin, Nucl. Phys. B279 (1987) 514.

[13] R. Banerjee, H. J. Rothe and K. D. Rothe, Phụs. Rev D55 (1997) 6339. 
[14] E. Fradkin, F. A. Schaposnik, Phys. Lett. B338 (1994) 253; K. Kondo, Prog. Theor. Phys. 94 (1995) 869.

[15] M. Gomes, L. C. Malacarne and A. J. da Silva, Phys. Lett. B439 (1998) 137.

[16] S. Olariu and I. I. Popescu. Rev. Mod. Phys. 57 (1985) 339.

[17) S. Ruijsenaars, Ann. Phys. (N.Y.) 146 (1983) 1.

(18) M. Peshkin and A. Tonomura, The Aharanov-Bohm Effect, Lecture Notes in Physics 340 Springer, Verlag, (1989).

[19] C. R. Hagen, Phys. Rev. Lett, 64 (1990) 503: C. R. Hagen, Phys. Rer. D41 (1990) $2015 ;$, C. R. Hagen, Phys. Rev. D48 (1993) 5935.

[20] C. R. Hagen and S. Ramaswamy, Phys. Rey D42 (1990) 3524.

[21] M. L. Horner and A. S. Goldhaber, Phys. Rev, D55 (1997) 5951 .

[22] O. Bargman and G. Lozano, Ann. Phys. (N.Y.) 220 (1994) 416.

[23] H. O. Giroti, N. Gomes, J. R. S. Nascimento, A. J. da Silya, Phys. Rev. D56 (1997) 3623.

[24] G. Amelino-Camelia and D. Bak, Phys. Lett. B 343 (1995) 231.

[25] S. I. Kim and C. Lee, Phys. Rex. D 55 (1997) 2227. S. A. Kim, Phys. Lett. B 343 (1995) 244.

[26] P. Giacconi, F. Maltoni, R. Soldati, hep-th/9706198.

[27] L. L. Foldy and S. A. Wouthuysen, Phys. Rex. 78(1950)78.

[28] J. M. C. Malbouisson, Estudos sobre o Limite Nä Relativistico en Teorios de Campos em $2+1$ Dimensôes, Tese de Doutorado, TFUSP, São Paulo, 1990.

[20] M. Gomes, J. M. C. Malbouisson, A. J. da Sila, Mod. Phys Leth. A11 (1996) 2825: Phys. Lett. A207 (1097) 373.

[30] A. Gomes, A. J. da Silva. Phys. Rex, D57 (1998) 3370.

[31] K. G. Wilson, Phys. Rev. B4 (1071) 374: W. e. Caswell and G. P. Lepage, Phys. Lett. B167 (1986) 437; G. P. Lepage, proceedings of TASI-89, 11989: T. Kinoshita and G. Lepage in "Quantum Electrodynamics", T. Kinoshita ed., World Seientific, Singapore, 1000. J. Polchinski, proceedings os TAS.02, J. Harvey and J. Polchinski eds. World Scientific. Singapore, 1993; A. V. Manohar, Proceedings of the International Winter School of Xuclear and Particle Physics, Schladming. Austria, 1996(hepph/9600222). 
[32] T. Lee, P. Oh, Ann. Phys. (N.Y.) 235 (1994) 413; H. Lo and J. Preskill, Phys. Rev. D48 (1993) 4821.

[33] T. T. Wu e C. N. Yang, Phys. Rev. D12 (1975) 3845.

[34] P. A. Horvathy, Phys. Rev. D33 (1986) 407.

[35] E. Verlinde, Proceedings of the International Collogium on Modern Quantum Field Theory, Bombay, January 1990.

[36] W. Witten, Nucl. Phys. B311 (1988) 46.

[37] M. G. Alford e F. Wilczek, Phys. Rev. Lett. 62 (1989) 1071.

[38] F. Wilczek e Y. S. Wu, Phys. Rev. Lett. 65 (1990) 13.

[39] M. G. Alford, J. March-Russel e F. Wilczek, Nucl. Phys. B337 (1990) 695.

[40] T. W. B. Kibble, J. Phys A9 (1976) 1387.

[41] A. Vilenkin, Phys. Rew. D23 (1981) 852:

[42] E. L. Feinberg, Sov. Phys. Usp. 5 (1963) 753; E. Corinaldesi and F. Rafeli, Am. J. Phys. 46 (1978) 1185; K. M. Purcell and W. C. Henneberger, Am. J. Phys. 46 (1978) 1255.

[43] M. Gomes and A. J. da Silva, Phys. Rev D34 (1986) 3916.

[44] J. A. Swieca, Fort. der Physik, 25 (1977) 303: S. Coleman, Phys. Rev. D11 (1975) 2088: S. Mandelstan, Phys. Rev. D11 (1975) 3026: M Bander, Phys. Rev. D13 (1976) 1566.

[45] F. A. Schaposnik, Phys. Lett. B356 (1995) 39: R. Banerjee and E.C. Marino, Nucl.Phys. B507 (1997) 501; E. C. Marino, Phys.Lett. B393 (1997) 383; R. Banerjee, Nucl.Phys. B465 (1996) 157.

[46] A. Karlhede et al, Phys. Lett. B186 (1987) 96.

[47] P. J. Arias. A. Restuccia. Phys. Lett B347 (1993) 1241: P. J. Arias, L. Leal and A. Restuccia. Phys. Lett B 367 (1996) 170.

[48] N. Bralić, E. Fradkin, V. Manias and F. A. Schaposnik, Nucl. Phys. B 446 (1995) 144.

[49] J. C. Le Guillou, E. Moreno, C. Núnez and F. A. Schaposnik, Nucl. Phys. B 484 (1997) 682. 
[\%0] N. Banerjee, R. Banerjee, S. Ghosh, Nucl. Phys. B 481 (1906) 421 .

[51] J. H. Lowenstein and D. Schroer, Phys. Rev. D6, (1972) 1553.

[52] M. Gomes, R. S. Mendes, R. F. Ribeiro and A. J. da Silva, Phys. Rev. D43, (1991) 3516.

[53] R. G. Amold, C. E. Carlson and F. Gross, Phys. Res, C21 (1980) 1426. Phys. Lett. B136 (1984) 38 .

[54] F. P. Devecchi, M. Fleck, H. O. Girotii, M. Gomes and A. J. da Silva, Ann. Phys. (N.Y.) $242(1995) 275$

[55] Existe uma certa controversia no uso da regulatiataya dimensional, devido a dif

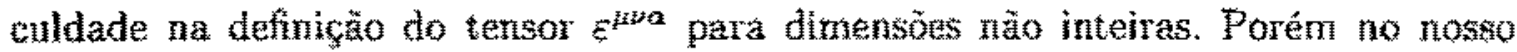
caso, nós iremos efetuar toda a álgebra tensorial em 3-dimensões e só iremos para $\mathrm{D}$ dimensöes no cálculo explicito da integral. Assim usaremos que as integracôes dimensionais em 1-Loop podem ser escritas na forma geral como

$$
\int \frac{d^{n} k}{(2 \pi)^{n}} \frac{\left(k^{2}\right)^{n}}{\left[k^{2}-C^{2}\right]^{b}}=(-1)^{n-a} \frac{i}{(4 \pi)^{n / 2}}\left[C^{2}\right]^{n / 2+a-b} \frac{\Gamma(b-a-n / 2) \Gamma(a+n / 2)}{\Gamma(n / 2) \Gamma(b)} .
$$

[56] H.C. Corben and J. Schwinger, Phys. Rev 58 (1902) 953.

[57] T. D. Lee and C. N. Yang, Phys. Rer. 128 (1902) 88.

[48] S. Ferrara and M. Porrati, Phys, Rev. D46 (1052) 350.

[59] L. Durand, P. C. DeCelles and r. B. Mar, Phys. Rev 126 (1962) 1882.

[60] K. J. Kim and Y. S. Tsai, Phys. Rev. D7 (1973) 3710.

[61] D. Bak and O. Bergman, Phys. Rex. D51 (1995) 1904.

[62] M. Gomes, L. C. Malacarne and ł. J. da Silva, Phys. Per. D59 04501

[63] R. Jackiw and S. Y. Pi, Phys.Rey. D42 (1990) 3000.

[64] F. Ferrari and L. Lazazzera, Int. J. Mod. Phys. A13 (1998)1773: hep-th/9712076:

(60) S. K. Paul and A. Khare, Phys Lett. B171 (1986) 244.

60 R. Gianvittorio, A. Restuccia and J. Stephany, Mod. Phys Lett. A6 (1991) 2121.

[67] J. R. S. do Nascinento, Estudo da Teoria de Chem-Simons no Gauge de Comlomb. Tese de Doutorado. IFUSP, São Paulo, 199é. 
[68] G. S. Adkins, Phys. Rex. D27 (1983) 1814.

[69] I. S. Gradshteyn and I. M. Ryzhik, Table of Integrals, Series, and Products, Academic Press, 1963. 